\title{
Representações parciais de grupos, seus domínios e o multiplicador de Schur parcial
}

\author{
Helder Geovane Gomes de Lima
}

TESE APRESENTADA

$\mathrm{AO}$

Instituto de Matemática e Estatística

DA

UniversidADE DE SÃo PAUlo

PARA

OBTENÇÃO DO TÍTULO

$\mathrm{DE}$

DOUTOR EM CIÊNCIAS

\author{
Programa: Matemática \\ Orientador: Prof. Dr. Mikhailo Dokuchaev
}

Durante o desenvolvimento deste trabalho

o autor recebeu auxílio financeiro da FAPESP. Processo 2009/53551-9.

São Paulo, março de 2014 


\section{Representações parciais de grupos, seus domínios e o multiplicador de Schur parcial}

Esta versão da tese contém as correções e alterações sugeridas pela Comissão Julgadora durante a defesa da versão original do trabalho, realizada em 28/03/2014. Uma cópia da versão original está disponível no

Instituto de Matemática e Estatística da Universidade de São Paulo.

Comissão Julgadora:

- Prof. Dr. Alveri Alves Sant'Ana - UFRGS

- Prof. Dr. Antonio Paques - UFRGS

- Prof. Dr. Eduardo do Nascimento Marcos - IME-USP

- Prof. Dr. Marcelo Muniz S. Alves - UFPR

- Prof. Dr. Mikhailo Dokuchaev (orientador) - IME-USP 
Dedico estre trabalho à memória de Boris Novikov. 


\section{Agradecimentos}

Gostaria de aproveitar este espaço para expressar a minha gratidão a todos que, de uma forma ou de outra, ajudaram a tornar esta tese possível.

Acima de tudo, sou muito grato à minha família - em especial à minha mãe, ao meu pai, e aos meus irmãos - por acreditarem em mim, me apoiarem e me incentivarem em todos os momentos, além de serem compreensivos com a minha ausência.

Também agradeço ao meu orientador Mikhailo Dokuchaev, por sua paciência e sua disposição para esclarecer as dúvidas que surgiram durante a minha formação, e pela inspiração para me dedicar e me tornar bom pesquisador.

Sou grato aos meus grandes amigos, aos que tive a oportunidade de conhecer nesse período, àqueles com quem joguei conversa fora, com quem troquei ideias, ou dúvidas, ou que gentilmente apontaram meus erros, que estiveram juntos em momentos marcantes, nos seminários, nos congressos, nas vésperas de provas, nas pizzarias, nos passeios e nos recitais, e também àqueles que mesmo a distância estão sempre comigo.

Agradeço ainda a cada um dos compositores cuja boa música me acompanha (quase) todos os dias, e à FAPESP pelo apoio financeiro.

Graças a cada um de vocês, pude sonhar e conseguir forças para batalhar pelos meus sonhos. 


\section{Resumo}

\section{LIMA, H. G. G. de. Representações parciais de grupos, seus domínios e o multiplicador de}

Schur parcial. 2014. 68 f. Tese (Doutorado) - Instituto de Matemática e Estatística, Universidade de São Paulo, São Paulo, 2014.

O multiplicador de Schur parcial de um grupo $G$ é um semigrupo inverso comutativo $p M(G)$ que, no estudo de representações parciais projetivas de grupos, desempenha um papel análogo ao do multiplicador de Schur clássico $M(G)$. Há uma descrição de $p M(G)$ como uma união de grupos abelianos, em que cada componente $p M_{D}(G)$ é formada por classes de equivalência de certas funções parciais (chamadas de conjuntos fatores parciais), as quais assumem valores em um corpo e têm como domínio um subconjunto $D \subseteq G \times G$. Os domínios $D$ formam um reticulado e foram caracterizados como os subconjuntos $\mathcal{T}$-invariantes de $G \times G$, em que $\mathcal{T}$ é um monoide específico atuando em $G \times G$. A componente total $p M_{G \times G}(G)$, que corresponde aos conjuntos fatores totalmente definidos, é particularmente interessante pois contém $M(G)$ como um de seus subgrupos e, além disso, qualquer outra componente é uma imagem epimorfa da componente total. Um dos objetivos deste trabalho é determinar a componente total do multiplicador de Schur parcial para algumas classes importantes de grupos, como os grupos diedrais, os grupos dicíclicos e os produtos de grupos cíclicos. Outro tópico que será abordado é a estrutura do reticulado dos domínios dos conjuntos fatores parciais, destacando-se propriedades daqueles que correspondem às representações parciais ditas elementares, as quais possuem um papel relevante na teoria. Provaremos que todo domínio pode ser representado em uma forma única como uma reunião de certos domínios indecomponíveis, que consistem de peças estruturais chamadas de blocos e domínios minimais. Também será determinada a estrutura dos domínios elementares e serão obtidos alguns invariantes numéricos do conjunto parcialmente ordenado dos domínios elementares. Como uma consequência dos resultados obtidos, serão caracterizados os grupos para os quais todos os domínios elementares são indecomponíveis. Além disso será feita uma aplicação da teoria de álgebras de semigrupos à álgebra parcial de grupo, que é uma álgebra responsável pelas representações parciais de grupos.

Palavras-chave: representações parciais projetivas de grupos, multiplicador de Schur parcial de um grupo, domínios elementares. 


\section{Abstract}

\section{LIMA, H. G. G. de. Partial group representations, their domains and the partial Schur}

multiplier. 2014. 68 f. Tese (Doutorado) - Instituto de Matemática e Estatística, Universidade de São Paulo, São Paulo, 2014.

The partial Schur multiplier of a group $G$ is a commutative inverse semigroup $p M(G)$ which, in the study of partial projective representations, plays a role analogous to the classical Schur multiplier $M(G)$. There is a description of $p M(G)$ as a union of abelian groups, in which each component $p M_{D}(G)$ is formed by the equivalence classes of certain partial functions (called partial factor sets), taking values in a field and having as its domain a subset $D \subseteq G \times G$. The domains $D$ form a lattice and were characterized as the $\mathcal{T}$-invariant subsets of $G \times G$, where $\mathcal{T}$ is a specific monoid acting on $G \times G$. The total component $p M_{G \times G}(G)$, which corresponds to the totally defined factor sets, is particularly interesting because it contains $M(G)$ as one of its subgroups and, moreover, any other component is an epimorphic image of the total component. One of the objectives of this work is to determine the total component of the partial Schur multiplier for some important classes of groups, such as the dihedral groups, the dicyclic groups and the products of cyclic groups. Another topic which will be considered is the structure of the lattice of domains of partial factor sets, emphasizing properties of those domains that correspond to the so-called elementary partial representations, which play a relevant role in the theory. We shall prove that each domain can be represented in a unique way as a union of certain indecomposable domains, where the latter consists of the so-called blocks and minimal domains. The structure of the elementary domains also will be determined, and some numerical invariants of the partially ordered set of the elementary domains will be given. As a consequence of the obtained facts, the groups whose elementary domains are indecomposable will be characterized. We will also give an application of the theory of semigroup algebras to the partial group algebra, an algebra which is responsible for partial group representations.

Keywords: partial projective representations of groups, partial Schur multiplier of a group, elementary domains. 


\section{Sumário}

Lista de Símbolos $\quad$ xi

Lista de Figuras $\quad$ xiii

Lista de Tabelas $\quad$ xv

$\begin{array}{ll}\text { Introdução } & 1\end{array}$

1 Preliminares $\mathbf{5}$

1.1 Ações Parciais de Grupos . . . . . . . . . . . . . . . . . . . . 5

1.2 Representações Parciais . . . . . . . . . . . . . . . . . . . . . . 7

1.3 Semigrupos e Reticulados . . . . . . . . . . . . . . . . . . 7

1.4 O Monoide $S(G)$ e Suas $\mathcal{J}$-classes $\ldots \ldots \ldots \ldots \ldots \ldots \ldots$

1.5 Representações Parciais Projetivas . . . . . . . . . . . . . . . . . . 12

2 Revisitando Alguns Fatos Conhecidos $\quad 17$

2.1 Decomposição da Álgebra Parcial de Grupo . . . . . . . . . . . . . . . . . . . . . . . . . . . . . . . . . . . .

2.2 Representações Parciais de Grau Finito . . . . . . . . . . . . . . . . . . 21

2.3 Interação entre Ações e Representações Parciais . . . . . . . . . . . . . . . . . 25

3 Domínios de Representações Parciais Projetivas $\quad 31$

3.1 Domínios Elementares . . . . . . . . . . . . . . . . . . . . . 31

3.2 Minimalidade e Indecomponibilidade . . . . . . . . . . . . . . . . . . 33

3.3 Decomposição de Domínios Elementares . . . . . . . . . . . . . . . . . . . . 37

3.4 Alguns Invariantes de $C(G) \ldots \ldots \ldots \ldots \ldots \ldots$

4 Multiplicador de Schur Parcial $\quad 45$

4.1 Grupos Diedrais . . . . . . . . . . . . . . . . . 48

4.2 Produto Direto de Grupos Cíclicos . . . . . . . . . . . . . . . . . . 50

4.3 Grupos Dicíclicos . . . . . . . . . . . . . . . . . . . 57

4.4 Grupos Infinitos . . . . . . . . . . . . . . . . . . . 60

4.4 Grupo Cíclico Infinito . . . . . . . . . . . . . . . . . . 60

4.4 .2 Grupo Diedral Infinito . . . . . . . . . . . . . . . . . . . . . . . . . . . . . . . . . 62

4.5 Sugestões para Pesquisas Futuras . . . . . . . . . . . . . . . . . . 64

$\begin{array}{ll}\text { A Diagramas de Domínios Elementares } & 65\end{array}$ 
B $S_{3}$-órbitas Efetivas de $C_{m} \times C_{n}$ 


\section{Lista de Símbolos}

\begin{tabular}{|c|c|c|c|}
\hline Símbolo & Página & Símbolo & Página \\
\hline $\mathcal{A} \rtimes_{\alpha} G$ & 6 & $\mathcal{V}_{A}$ & 21 \\
\hline$A / \operatorname{St} A$ & 12 & $X(G)$ & 51 \\
\hline$C(G)$ & 14 & $Y(e)$ & 51 \\
\hline$D_{A}$ & 32 & & \\
\hline $\operatorname{cls}(\sigma)$ & 15 & & \\
\hline $\operatorname{dom} \sigma$ & 13 & & \\
\hline$E(S)$ & 9 & & \\
\hline$\Gamma(G)$ & 21 & & \\
\hline$\Gamma_{\text {fin }}(G)$ & 22 & & \\
\hline $\mathcal{G}(\mathcal{S}(G))$ & 22 & & \\
\hline$I_{a}$ & 8 & & \\
\hline$J_{a}$ & 8 & & \\
\hline $\mathcal{J}_{a}$ & 8 & & \\
\hline $\mathcal{J}_{A}$ & 12 & & \\
\hline$K[S]$ & 8 & & \\
\hline$K_{0}[S]$ & 8 & & \\
\hline $\operatorname{ker}(S)$ & 9 & & \\
\hline$L_{D}$ & 15 & & \\
\hline$\lambda_{\Delta}$ & 23 & & \\
\hline$N_{D}$ & 14 & & \\
\hline$o_{n}(G)$ & 40 & & \\
\hline$p m(G)$ & 14 & & \\
\hline$p M(G)$ & 14 & & \\
\hline$p m_{D}^{\prime}(G)$ & 14 & & \\
\hline$s(G, D)$ & 14 & & \\
\hline$S^{0}(H ; \mathcal{I}, \Delta ; P)$ & 10 & & \\
\hline$M(A ; \mathcal{I}, \Delta ; P)$ & 10 & & \\
\hline $\operatorname{Proj} M$ & 13 & & \\
\hline $\mathcal{S}(G)$ & 11 & & \\
\hline$S_{G}$ & 50 & & \\
\hline St $A$ & 12 & & \\
\hline $\mathcal{T}$ & 13 & & \\
\hline $\mathcal{T}(G)$ & 40 & & \\
\hline$T_{G}$ & 50 & & \\
\hline
\end{tabular}




\section{Lista de Figuras}

1.1 Ilustração da definição de ação parcial de grupo. . . . . . . . . . . . . . . . 5

1.2 Restrição de uma ação total $\beta$ de um grupo em $\mathcal{B}$ a uma ação parcial $\alpha$ em $\mathcal{A}$. . . . 6

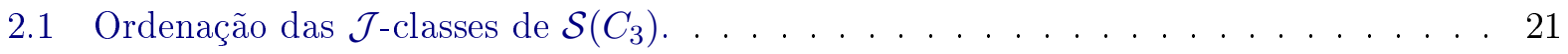

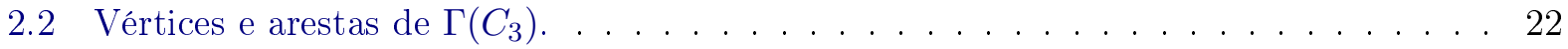

4.1 Representantes das órbitas efetivas de $S_{3}$ em $C_{9} \times C_{9}$, em que $C_{9}=\left\langle a \mid a^{9}=1\right\rangle . \quad$. $\quad 46$

A.1 Ordem parcial dos domínios elementares de $C_{2}=\left\langle a \mid a^{2}=1\right\rangle \ldots \ldots \ldots \ldots$

A.2 Ordem parcial dos domínios elementares de $C_{3}=\left\langle a \mid a^{3}=1\right\rangle \ldots \ldots \ldots \ldots$. . . . 65

A.3 Ordem parcial dos domínios elementares de $C_{4}=\left\langle a \mid a^{4}=1\right\rangle \ldots \ldots \ldots \ldots$. . . . 65

A.4 Ordem parcial dos domínios elementares de $V=\left\langle a, b \mid a^{2}=b^{2}=(a b)^{2}=1\right\rangle \ldots \ldots 6$

A.5 Ordem parcial dos domínios elementares de $C_{5}=\left\langle a \mid a^{5}=1\right\rangle \ldots \ldots \ldots$. . . . . 66

A.6 Ordem parcial dos domínios elementares de $C_{6}=\left\langle a \mid a^{6}=1\right\rangle \ldots \ldots \ldots$. . . . . . 66

A.7 Ordem parcial dos domínios elementares de $S_{3}=\left\langle a, b \mid a^{3}=b^{2}=(a b)^{2}=1\right\rangle \ldots \ldots 6$ 


\section{Lista de Tabelas}

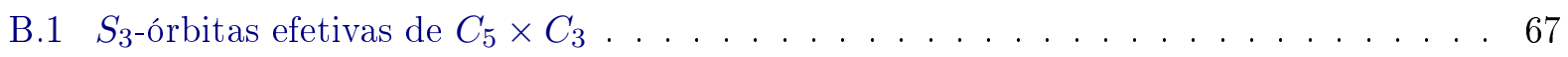

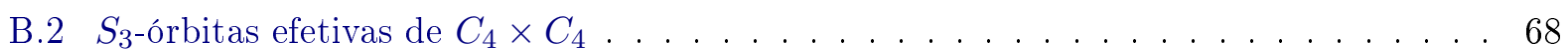




\section{Introdução}

O conceito de ação parcial foi elaborado na área de álgebra de operadores, nos artigos [25, 32, $26,27]$. Exel apresentou uma correspondência biunívoca entre as ações parciais de um grupo $G$ e as ações (globais) de um semigrupo inverso $\mathcal{S}(G)$ que pode ser construído a partir daquele grupo (ver Definição 1.4.1). Posteriormente, o estudo de ações e representações parciais continuou em diferentes frentes de trabalho, investigando-se por exemplo:

- Qual é a estrutura da álgebra parcial de grupo cuja teoria de representações corresponde à teoria de representações parciais do grupo? Isso foi discutido em [16, 23], para grupos finitos.

- No caso de grupos infinitos, como descrever a estrutura das representações parciais irredutíveis (ou indecomponíveis) de grau finito em termos de representações usuais? Esse problema foi tratado em [24].

- Em que circunstâncias uma ação parcial de grupo é a restrição de alguma ação global, ou seja, quando ela é globalizável? O estudo disso iniciou-se em [1, 2, 39, 30, 15], e há informações adicionais em [14].

- Qual a estrutura do produto cruzado parcial, e quais são as suas propriedades? Ver, por exemplo, [14].

- E se em vez de ações parciais de grupos forem consideradas generalizações para monoides, grupoides, ou álgebras de Hopf? Quando existirá uma globalização? Isso foi abordado em $[33,28,9,10,5,6]$.

- Ao globalizar uma ação parcial de grupo ou de monoide sobre certo espaço topológico, que propriedades do espaço são transferidas para a globalização? Tal questão foi discutida em $[2,33]$.

Além das questões acima, foi estudado o problema do isomorfismo para álgebras parciais de grupos [14] e foi introduzida em [17] uma teoria de Galois de ações parciais, a qual continuou a ser estudada em trabalhos como [11,36]. Foram desenvolvidas ainda diversas aplicações à teoria de autômatos [22] e álgebras de Leavitt [29], além de ter sido publicada uma série de trabalhos relacionados ao assunto na área de $C^{*}$-álgebras (há uma discussão desses trabalhos em [14]). Também desenvolveu-se uma teoria de representações parciais projetivas [14], uma teoria cohomológica baseada em ações parciais em [18] e foram pesquisados outros assuntos sobre ações e co-ações de álgebras de Hopf [4, 3, 7, 8, 12].

O multiplicador de Schur parcial $p M(G)$ de um grupo $G$ foi introduzido em [19] e [20], juntamente com a noção de uma representação parcial projetiva de um grupo sobre um corpo. Ele é uma generalização do multiplicador de Schur usual $M(G)$ e aparece naturalmente no estudo de uma nova teoria de cohomologia baseada em ações parciais. Ao contrário de sua versão clássica, que é um grupo, este multiplicador tem a estrutura de um semirreticulado de grupos abelianos $p M_{D}(G)$, denominados componentes, indexados por certos subconjuntos $D \subseteq G \times G$ (ver Teorema 1.5.8). Fixando-se um corpo $K$, cada componente $p M_{D}(G)$ é formada por funções parcialmente definidas $\sigma: G \times G \rightarrow K$, que têm $D$ como domínio e que são conhecidas como conjuntos fatores parciais. Esses conjuntos fatores parciais estão associados às representações parciais projetivas conforme a Definição 1.5.5. 
Sabe-se que os domínios dos conjuntos fatores parciais formam um reticulado, e eles foram caracterizados em [19, Corollary 7] como os subconjuntos $\mathcal{T}$-invariantes de $G \times G$, em que $\mathcal{T}$ é um monoide específico atuando em $G \times G$ (ver (1.3) e (1.5)). Um dos objetivos deste trabalho é descrever a estrutura destes domínios, e também dos domínios que estão associados a certas representações parciais conhecidas como elementares (ver (3.1)).

A componente $p M_{G \times G}(G)$ do multiplicador de Schur parcial $p M(G)$ (que corresponde aos conjuntos fatores totalmente definidos) é particularmente importante, pois conforme [19] o multiplicador de Schur usual $M(G)$ é um de seus subgrupos e, além disso, de acordo com [21, Corollary 5.8 (iv)] qualquer componente é uma imagem epimorfa da componente total. Alguns trabalhos recentes forneceram uma descrição para a componente total $p M_{G \times G}(G)$ do multiplicador de Schur parcial $p M(G)$ sobre corpos algebricamente fechados nos casos em que $G$ é um grupo cíclico finito [21, Corollary 6.4] ou um 2-grupo abeliano elementar $C_{2}^{n}$ [34], e também no caso em que $G=S_{3} \simeq D_{6}$ [38, Lemma 3.10].

O último capítulo deste trabalho será dedicado ao estudo da componente total do multiplicador de Schur parcial para algumas outras classes de grupos importantes. Em particular, uma generalização de [38, Lemma 3.10] para os grupos diedrais $D_{2 m}$ será apresentada no Teorema 4.1 .1 e [21, Corollary 6.4] será generalizado para o produto direto $C_{m} \times C_{n}$ de grupos cíclico finitos no Corolário 4.2.6, e para o grupo cíclico infinito no Corolário 4.4.3. Além disso, a componente total também é descrita no caso dos grupos dicíclicos. Estes resultados foram obtidos em colaboração com H. Pinedo.

\section{Contribuições}

As principais contribuições deste trabalho são as seguintes:

- Descrição da estrutura do semirreticulado de domínios e dos domínios elementares;

- Descrição da componente total do multiplicador de Schur parcial para algumas famílias de grupos, a saber, grupos diedrais, grupos dicíclicos, produtos de grupos cíclicos, bem como para o grupo cíclico de ordem infinita e o grupo diedral de ordem infinita;

- Obtenção de um análogo de [20, Theorem 3] sobre a relação entre as categorias de ações parciais e a categoria de representações parciais no Teorema 2.3.3;

- Obtenção de uma demonstração alternativa para a estrutura da álgebra parcial de grupos finitos;

- Preenchimento de uma lacuna na demonstração de um resultado de [24] (vide Teorema 2.2.3 e Proposição 2.2.4 ).

\section{Organização do Trabalho}

No primeiro capítulo, serão apresentados os conceitos básicos de ações e representações parciais, bem como algumas definições e notações que são comuns na teoria de semigrupos. Além disso, o capítulo introduz as noções de representações parciais projetivas, os conjuntos fatores parciais e o multiplicador de Schur parcial, e relembra alguns fatos relacionados ao multiplicador parcial de Schur que serão utilizados ao caracterizar a componente total do multiplicador de Schur parcial para certas famílias de grupos.

O capítulo seguinte discute alguns resultados que foram apresentados originalmente nos artigos $[16,24,20]$, começando pela exposição de uma demonstração alternativa para a decomposição da álgebra parcial de grupo em soma de álgebras matriciais sobre álgebras de subgrupos. Em um segundo momento, é demonstrada uma proposição cujo propósito é completar uma lacuna existente na prova de [24, Theorem 2.2], sobre as representações parciais de grau finito de grupos. O capítulo se encerra com a discussão da interação entre ações parciais e representações parciais em álgebras. 
A partir do Capítulo 3 são consideradas representações parciais projetivas. Nas primeiras seções do terceiro capítulo são demonstradas algumas propriedades dos domínios elementares, e é dada uma descrição dos domínios minimais e indecomponíveis. Além disso, prova-se que todo domínio pode ser decomposto de forma única como uma união de certos domínios indecomponíveis, determina-se a estrutura dos domínios elementares e obtêm-se alguns invariantes numéricos do conjunto parcialmente ordenado dos domínios elementares. Por meio desses resultados, caracterizam-se os grupos para os quais todos os domínios elementares são indecomponíveis. Estes resultados foram obtidos em colaboração com H. Pinedo e com o professor M. Dokuchaev.

No Capítulo 4 será descrita a componente total $p M_{G \times G}(G)$ do multiplicador de Schur parcial $p M(G)$ para algumas famílias de grupos $G$, e serão indicados alguns caminhos que poderão ser seguidos em pesquisas futuras.

Finalmente, o Apêndice A mostra como são os domínios elementares para alguns grupos de ordem pequena e o Apêndice B ilustra como são as $S_{3}$-órbitas efetivas de produtos de grupos cíclicos finitos, e também a lista de representantes considerada no Exemplo 4.2.4. 


\section{Capítulo 1}

\section{Preliminares}

Neste capítulo são apresentadas definições e resultados que serão úteis para a compreensão dos capítulos posteriores. Ao longo do texto, salvo indicação em contrário, $K$ sempre denotará um corpo, $K^{*}$ o conjunto de seus elementos diferentes de zero e $G$ um grupo.

\subsection{Ações Parciais de Grupos}

O conceito de ação parcial de grupo será definido como em [27, 15]:

Definição 1.1.1. Uma ação parcial de um grupo $G$ em um conjunto $X$ é uma coleção de bijeções $\alpha_{g}: \mathcal{D}_{g^{-1}} \rightarrow \mathcal{D}_{g}$ entre subconjuntos $\mathcal{D}_{g} \subseteq X$, para cada $g \in G$, tal que:

(i) $\mathcal{D}_{1}=X$ e $\alpha_{1}=\operatorname{Id}_{X}$ (1 é o elemento neutro de $\left.G\right)$;

(ii) Para quaisquer $g, h \in G$ :

(a) $\alpha_{g}\left(\mathcal{D}_{g^{-1}} \cap \mathcal{D}_{h}\right)=\mathcal{D}_{g} \cap \mathcal{D}_{g h}$

(b) $\alpha_{g}\left(\alpha_{h}(x)\right)=\alpha_{g h}(x)$, para todo $x \in \mathcal{D}_{h^{-1}} \cap \mathcal{D}_{(g h)^{-1}}$.

Há uma definição alternativa segundo a qual uma ação parcial de grupo é uma função parcial de $G \times X$ em $X$ que satisfaz certas propriedades (para detalhes, consultar [30]). De forma mais geral, pode-se considerar também ações parciais de monoides como foi feito em [33].

As condições acima podem ser melhor visualizadas por meio da Figura 1.1. Quando há diversas

Figura 1.1: Ilustração da definição de ação parcial de grupo.

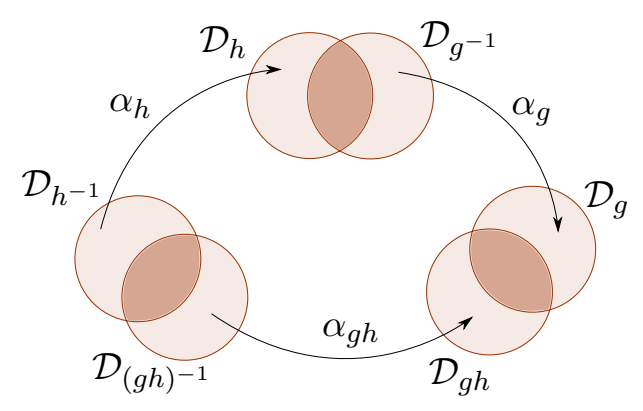

ações parciais de um mesmo grupo sendo consideradas, é interessante poder compará-las, e identificar aquelas que são, em sua essência, uma mesma ação parcial. A definição abaixo descreve essa identificação de forma mais precisa:

Definição 1.1.2. Sejam $\alpha$ e $\alpha^{\prime}$ ações parciais de um grupo $G$ nos conjuntos $X$ e $X^{\prime}$ dadas por $\left\{\alpha_{g}: \mathcal{D}_{g^{-1}} \rightarrow \mathcal{D}_{g} \mid g \in G\right\}$ e $\left\{\alpha_{g}^{\prime}: \mathcal{D}_{g^{-1}}^{\prime} \rightarrow \mathcal{D}_{g}^{\prime} \mid g \in G\right\}$, respectivamente. Um morfismo ${ }^{1}$ de $\alpha$ em $\alpha^{\prime}$ é uma função $\varphi: X \rightarrow X^{\prime}$ tal que, para todo $g \in G$ :

\footnotetext{
${ }^{1}$ Em [33] os morfismos de ações parciais de monoides foram denominados aplicações equivariantes.
} 
(i) $\varphi\left(\mathcal{D}_{g}\right) \subseteq \mathcal{D}_{g}^{\prime}$;

(ii) $\alpha_{g}^{\prime}(\varphi(x))=\varphi\left(\alpha_{g}(x)\right)$, para todo $x \in \mathcal{D}_{g^{-1}}$, ou seja, o diagrama

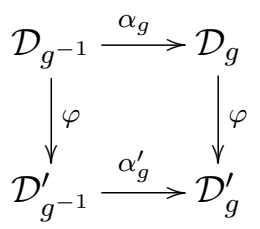

é comutativo.

No caso de $X$ ser uma $K$-álgebra associativa $\mathcal{A}$, em que $K$ é um corpo, define-se uma ação parcial de $G$ em $\mathcal{A}$ exigindo-se, além das condições presentes na Definição 1.1.1, que os domínios $\mathcal{D}_{g}$ sejam ideais de $\mathcal{A}$ e que as aplicações $\alpha_{g}(g \in G)$ sejam isomorfismos de álgebras (ver [15]). Neste caso, um morfismo entre ações parciais de $G$ nas álgebras $\mathcal{A}$ e $\mathcal{A}^{\prime}$ é definido como um homomorfismo de álgebras $\varphi: \mathcal{A} \rightarrow \mathcal{A}^{\prime}$ satisfazendo as propriedades da Definição 1.1.2. O morfismo $\varphi$ será chamado de epimorfismo se cada restrição $\varphi: \mathcal{D}_{g} \rightarrow \mathcal{D}_{g}^{\prime}$ for um epimorfismo de álgebras, monomorfismo se tais restrições forem monomorfismos de álgebras e isomorfismo se elas forem isomorfismos de álgebras. Neste último caso, as ações parciais em questão serão denominadas isomorfas. ${ }^{2}$

Conforme [15, Theorem 4.5], uma ação parcial de $G$ em uma álgebra $\mathcal{A}$ (com unidade) pode ser vista como a restrição de uma ação total (ou seja, é globalizável) se, e somente se, cada ideal $\mathcal{D}_{g}$ for uma álgebra com unidade. A obtenção de ações parciais a partir de ações totais é feita de forma bastante natural, como é ilustrado pela Figura 1.2. A definição precisa não será utilizada neste texto, mas pode ser consultada em [15].

Figura 1.2: Restrição de uma ação total $\beta$ de um grupo em $\mathcal{B}$ a uma ação parcial $\alpha$ em $\mathcal{A}$.

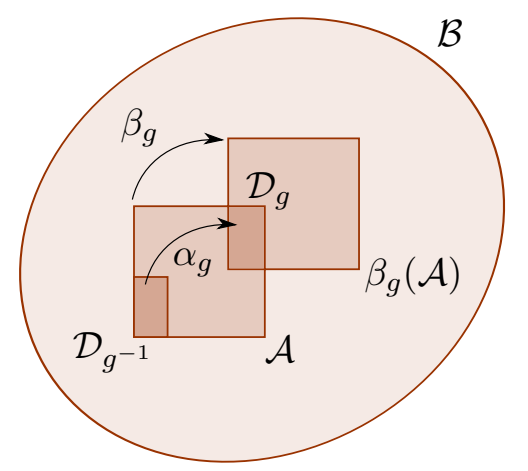

Para entender a relação entre as ações e as representações parciais de grupos em álgebras que serão definidas na próxima seção, foi utilizada a seguinte noção em [15]:

Definição 1.1.3. Seja $\alpha$ uma ação parcial de um grupo $G$ em uma álgebra $\mathcal{A}$. O anel de grupo skew correspondente é definido como

$$
\mathcal{A} \rtimes_{\alpha} G=\left\{\sum_{g \in G} a_{g} \delta_{g}: a_{g} \in \mathcal{D}_{g}\right\},
$$

em que a soma é definida termo a termo, e o produto é determinado por

$$
\left(a_{g} \delta_{g}\right) \cdot\left(b_{h} \delta_{h}\right)=\alpha_{g}\left(\alpha_{g^{-1}}\left(a_{g}\right) b_{h}\right) \delta_{g h}
$$

Quando a ação parcial é globalizável, esse anel é associativo.

\footnotetext{
${ }^{2} \mathrm{Em}[15]$ elas foram denominadas equivalentes.
} 


\subsection{Representações Parciais}

Definição 1.2.1. Chama-se de representação parcial de $G$ em uma álgebra $\mathcal{A}$ (com unidade) a qualquer aplicação $\phi: G \rightarrow \mathcal{A}$ que verifique as seguintes propriedades:

(i) $\phi(1)=1_{\mathcal{A}}$

(ii) $\phi(g) \phi(h) \phi\left(h^{-1}\right)=\phi(g h) \phi\left(h^{-1}\right)$

(iii) $\phi\left(g^{-1}\right) \phi(g) \phi(h)=\phi\left(g^{-1}\right) \phi(g h)$

Em particular, toda representação (usual) de grupo é também uma representação parcial.

A cada grupo $G$ e a cada corpo $K$ associou-se em [16] uma $K$-álgebra com unidade que cumpre um papel análogo ao da álgebra de grupo no estudo das representações usuais:

Definição 1.2.2. A álgebra parcial do grupo $G$ sobre $K$, denotada por $K_{\text {par }}(G)$, é a álgebra gerada pelo conjunto de símbolos $\{[g]: g \in G\}$, sujeitos às seguintes relações:

(i) $[1]=1$

(ii) $\left[g^{-1}\right][g][h]=\left[g^{-1}\right][g h]$

(iii) $[g][h]\left[h^{-1}\right]=[g h]\left[h^{-1}\right]$

para quaisquer $g, h \in G$.

Uma das razões para o interesse no estudo de $K_{\mathrm{par}}(G)$ é que as representações (usuais) dessa álgebra correspondem biunivocamente às representações parciais de $G$, caso ele seja finito. O estudo da estrutura da álgebra parcial de grupo mencionado na introdução envolveu, entre outras coisas, o problema do isomorfismo no contexto de álgebras parciais de grupos (ver [16] e [23]). Nos artigos mencionados, os autores mostraram como um anel parcial $R_{\text {par }}(G)$ pode ser decomposto como soma direta de anéis de matrizes $M_{m}(R H)$, com $H$ variando entre os subgrupos de $G$. No Teorema 2.1.2 será apresentada uma demonstração alternativa para essa decomposição de $K_{\text {par }}(G)$, utilizando-se da teoria de álgebra de semigrupos.

Definição 1.2.3. Sejam $\pi: G \rightarrow \mathcal{B}$ e $\pi^{\prime}: G \rightarrow \mathcal{B}^{\prime}$ representações parciais de $G$. Um morfismo de $\pi$ $e m \pi^{\prime}$ é um homomorfismo de álgebras $\varphi: \mathcal{B} \rightarrow \mathcal{B}^{\prime}$ tal que o diagrama

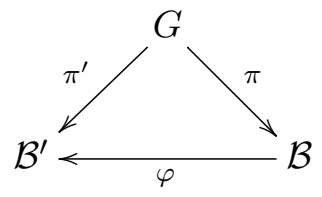

é comutativo, isto é, para todo $g \in G, \pi^{\prime}(g)=\varphi(\pi(g))$.

\subsection{Semigrupos e Reticulados}

Nesta seção, serão fixadas algumas notações que são usuais na teoria de semigrupos e de álgebras de semigrupos e destacados alguns fatos que serão úteis posteriormente.

Um semigrupo $S$ é um conjunto no qual está definida uma operação binária associativa. Se essa operação binária possui um elemento neutro, denotado por 1, então $S$ é chamado de monoide. Dado um semigrupo $S$, o monoide obtido de $S$ por meio da adjunção de uma unidade é o conjunto

$$
S^{1}= \begin{cases}S, & \text { se } S \text { tem elemento neutro, } \\ S \cup\{1\}, & \text { caso contrário, }\end{cases}
$$


Para todo $x \in S \cup\{1\}$, define-se $1 x=x 1=x$. De forma análoga, pode-se obter um semigrupo com zero a partir de $S$ :

$$
S^{0}= \begin{cases}S & \text { se } S \text { tem zero, } \\ S \cup\{0\}, & \text { caso contrário. }\end{cases}
$$

Aqui, se $x \in S \cup\{0\}$, define-se $0 x=x 0=0$.

Dado $a \in S$, serão utilizadas ainda as seguintes notações:

- O ideal principal de $S$ gerado por a é o conjunto $S^{1} a S^{1}=S a S \cup a S \cup S a \cup\{a\}$ e será denotado por $J_{a}$;

- A $\mathcal{J}$-classe de $a$ é formada pelos elementos $b$ tais que $J_{b}=J_{a}$ (ou seja, geradores de $J_{a}$ ) e é denotada por $\mathcal{J}_{a}$. Em outras palavras, $a, b \in S$ estão em uma mesma $\mathcal{J}$-classe se, e somente se, $S^{1} a S^{1}=S^{1} b S^{1}$;

- $I_{a}$ é o conjunto dos elementos de $J_{a}$ que não geram $J_{a}$ como um ideal de $S$. Assim, $I_{a}=J_{a} \backslash \mathcal{J}_{a}$ e $\mathcal{J}_{a}=J_{a} \backslash I_{a}$. Note que $I_{a}$ é vazio exatamente no caso em que $J_{a}$ é um ideal minimal de $S$, e quando isso não ocorre, $I_{a}$ é um ideal de $S$;

- As $\mathcal{J}$-classes serão ordenadas como em [13, p. 48]:

$$
\mathcal{J}_{a} \leq \mathcal{J}_{b} \text { se, e somente se, } J_{a} \subseteq J_{b} \text {. }
$$

Diz-se que $S$ é um semigrupo inverso, se para cada $a \in S$ existe um único $b \in S$ tal que $a b a=a$ e $b a b=b$. Neste caso, $b$ é chamado de inverso de $a$ e denotado por $a^{-1}$.

Lema 1.3.1. Se $S$ é um semigrupo, então:

(i) $\mathcal{J}_{a b} \leq \mathcal{J}_{a}$ e $\mathcal{J}_{a b} \leq \mathcal{J}_{b}$, para quaisquer $a, b \in S$;

(ii) Se $S$ é inverso, $\mathcal{J}_{a}=\mathcal{J}_{a^{-1}}$, para todo $a \in S$.

Demonstração. Tem-se $J_{a b}=S^{1} a b S^{1} \subseteq S^{1} a S^{1}=J_{a}$ e também $J_{a b}=S^{1} a b S^{1} \subseteq S^{1} b S^{1}=J_{b}$. Assim, se $S$ é inverso,

$$
\mathcal{J}_{a}=\mathcal{J}_{a\left(a^{-1} a\right)} \leq \mathcal{J}_{a^{-1} a} \leq \mathcal{J}_{a^{-1}}
$$

e a igualdade segue por simetria.

Definição 1.3.2. Seja $S$ um semigrupo. A álgebra de semigrupo de $S$ sobre $K$, denotada por $K[S]$, é o $K$-espaço vetorial com base $S$ munido de uma multiplicação que estende naturalmente a operação de $S$. Mais precisamente, $K[S]$ é o conjunto de todas as somas formais $\alpha=\sum_{s \in S} \alpha_{s} s$, em que apenas uma quantidade finita dos coeficientes $\alpha_{s} \in K$ é diferente de zero. A soma é dada por

$$
\sum_{s \in S} \alpha_{s} s+\sum_{s \in S} \beta_{s} s=\sum_{s \in S}\left(\alpha_{s}+\beta_{s}\right) s
$$

e o produto por

$$
\left(\sum_{s \in S} \alpha_{s} s\right) \cdot\left(\sum_{s \in S} \beta_{s} s\right)=\sum_{s \in S} \gamma_{s} s
$$

em que $\gamma_{s}=\sum_{u, v \in S, u v=s} \alpha_{u} \beta_{v}$.

Se $S$ possui zero $\theta$, define-se também a álgebra de semigrupo contraída de $S$ sobre $K$ como o quociente de álgebras $K_{0}[S]=K[S] / K \theta$, em que $K \theta=\{\alpha \theta \mid \alpha \in K\}$.

O Lema 1.3.4 destacará a relação entre a álgebra de semigrupo e a álgebra de semigrupo contraída. Para isso, tenha em mente a definição de quociente de semigrupos por ideais: 
Definição 1.3.3. Seja $I$ um ideal do semigrupo $S$ e considere a congruência $\sim_{I}$ definida por

$$
a \sim_{I} b \Leftrightarrow a=b \text { ou }\{a, b\} \subseteq I .
$$

Define-se o semigrupo quociente $S / I$ como sendo $S / \sim_{I}$.

Verifica-se que $S / I$ é isomorfo ao semigrupo $(S \backslash I) \cup\{0\}$ (assuma que $0 \notin S \backslash I$ ) no qual o produto de elementos arbitrários $a$ e $b$ é dado em termos de seu produto em $S$, da seguinte forma:

$$
a \cdot b= \begin{cases}a b, & \text { se } a, b, a b \in S \backslash I, \\ 0, & \text { caso contrário }\end{cases}
$$

Lema 1.3.4. Seja $S$ um semigrupo. Então:

- [35, Chapter 4, Corollary 8] Se I é um ideal de S tal que a álgebra de semigrupo K[I] possui unidade e, então $K[S] \simeq K[I] \oplus K_{0}[S / I]$, sendo o isomorfismo dado por $x \mapsto x e+\varphi(x), x \in$ $K[S]$, em que $\varphi$ é a aplicação natural de $K[S] \rightarrow K[S / I]$.

- [35, Chapter 4, Corollary 9] Se $S$ tem zero, então $K[S] \simeq K \oplus K_{0}[S]$.

Observação 1.3.5. Seja $S$ um semigrupo com zero. Segue do segundo item do Lema 1.3.4 que a existência de uma unidade em $K[S]$ equivale à existência de uma unidade em $K_{0}[S]$. Além disso, se $I$ é um ideal não-nulo de $S$ tal que a álgebra de semigrupo $K[I]$ (ou $K_{0}[I]$ ) possui unidade, então

$$
K_{0}[S] \simeq \frac{K[S]}{K \theta} \simeq \frac{K[I] \oplus K_{0}[S / I]}{K \theta} \simeq \frac{K[I]}{K \theta} \oplus K_{0}[S / I] \simeq K_{0}[I] \oplus K_{0}[S / I] .
$$

Se $S$ não possui zero, será considerado que $K_{0}[S]=K S$.

Definição 1.3.6. O núcleo de um semigrupo $S$ é definido como a interseção de todos os seus ideais bilaterais não vazios, e será denotado por $\operatorname{ker}(S)$.

Note que o núcleo de $S$ é também um ideal, e se $0 \in S$ então $\operatorname{ker}(S)=\{0\}$.

Definição 1.3.7. Um semigrupo $S$ é dito simples se não contém propriamente nenhum ideal bilateral. Analogamente, um semigrupo $S$ com 0 é dito 0 -simples se o seu único ideal bilateral próprio é $\{0\}$ e sua multiplicação não é nula $\left(S^{2} \neq\{0\}\right)$.

Entre os semigrupos simples (e os 0-simples) há um tipo especial que também é de grande importância na teoria de semigrupos. No que segue, $E(S)=\left\{e \in S \mid e^{2}=e\right\}$ denotará o conjunto dos idempotentes do semigrupo $S$.

Definição 1.3.8. Um idempotente $e$ de um semigrupo $S$ é dito primitivo se para todo idempotente $f$ não-nulo, $e f=f e=e$ implica $e=f$ ou $e=0$.

Definição 1.3.9. Um semigrupo $S$ é dito completamente simples (completamente 0 -simples) se $S$ é simples (0-simples) e tem algum idempotente primitivo.

A estrutura destes semigrupos ficará mais clara quando for introduzida a notação da Definição 1.3.14. Antes disso, será considerado um conceito que ajuda a entender como os semigrupos (0-)simples aparecem de forma natural "dentro" de outros semigrupos.

Definição 1.3.10. Dado $a \in S$, o semigrupo quociente $J_{a} / I_{a}$, ou simplesmente $J_{a}$ se $I_{a}=\emptyset$, é chamado de fator principal correspondente a $a$.

Observação 1.3.11. Note que se $I_{a} \neq \emptyset$, o fator principal $J_{a} / I_{a}=\mathcal{J}_{a} \cup\{0\}=\mathcal{J}_{a}^{0}$. Além disso, sabe-se por [13, Lemma 2.39] que em um semigrupo qualquer, todo fator principal é de um dos seguintes tipos: 
(i) Simples (se for o núcleo do semigrupo)

(ii) 0-simples

(iii) De ordem dois e com multiplicação nula

Quando a terceira condição não ocorre, o semigrupo em questão recebe um nome especial.

Definição 1.3.12. Um semigrupo $S$ é dito semissimples se nenhum de seus fatores principais possui multiplicação nula, isto é, se todos eles são 0-simples ou simples. Analogamente, se todo fator principal de $S$ é completamente simples ou completamente 0 -simples, $S$ é denominado completamente semissimples.

O seguinte fato também é conhecido da teoria de semigrupos:

Lema 1.3.13[13, Corollary 2.56]. Todo semigrupo finito simples (0-simples) é completamente simples (completamente 0-simples). ${ }^{3}$

Pelo teorema de Rees-Sushkevich (ver [13]), todos os semigrupos completamente 0-simples são descritos pela seguinte construção:

Definição 1.3.14. Dados um grupo $H$, conjuntos $\mathcal{I}$ e $\Delta$ não vazios (e não necessariamente finitos), um símbolo 0 e uma matriz $P=\left(p_{\lambda, i}\right)_{(\lambda, i) \in \Delta \times \mathcal{I}}$ com entradas em $H \cup 0$ e pelo menos um elemento não-nulo em cada linha e cada coluna, a notação $S^{0}(H ; \mathcal{I}, \Delta ; P)$ será utilizada para denotar o semigrupo $(\mathcal{I} \times H \times \Delta) \cup\{0\}$ em que o produto é definido por

$$
(i, g, \lambda) \cdot(j, h, \mu)= \begin{cases}\left(i, g p_{\lambda, j} h, \mu\right) & \text { se } p_{\lambda, j} \neq 0, \\ 0 & \text { caso contrário }\end{cases}
$$

$\mathrm{e}$

$$
0 \cdot(i, g, \lambda)=(i, g, \lambda) \cdot 0=0 \cdot 0=0 .
$$

Se $|\mathcal{I}|=m$ e $|\Delta|=n$, a notação será simplificada para $S^{0}(H ; m, n ; P)$.

De forma similar, todos os semigrupos completamente simples têm a forma $S(H ; \mathcal{I}, \Delta ; P)$, como na construção anterior, com a diferença de que $P$ é uma matriz com entradas em $H$.

O semigrupo $S^{0}(H ; \mathcal{I}, \Delta ; P)$ também pode ser representado por um conjunto de matrizes de dimensão $|\mathcal{I}| \times|\Delta|$ sobre $H \cup\{0\}$ com no máximo uma entrada diferente de zero, e com $a \cdot b=a P b$, para todo $a, b \in S^{0}(H ; \mathcal{I}, \Delta ; P)$, em que o lado direito é um produto usual de matrizes. De forma similar, define-se a seguinte álgebra:

Definição 1.3.15. Dados uma $K$-álgebra $A$, conjuntos $\mathcal{I}$ e $\Delta$ não vazios (e não necessariamente finitos), e uma matriz $P$ de dimensão $|\Delta| \times|\mathcal{I}|$ com entradas em $A$, a notação $\mathfrak{M}(A ; \mathcal{I}, \Delta ; P)$ será utilizada para denotar a álgebra de todas as matrizes de dimensão $|\mathcal{I}| \times|\Delta|$ sobre $A$ com uma quantidade finita de entradas não-nulas, em que a adição e a multiplicação por escalar são as usuais e o produto de $A$ e $B$ é definido por $A \cdot B=A P B$ (o lado direito é um produto usual de matrizes). Se $|\mathcal{I}|=m$ e $|\Delta|=n$, a notação será simplificada para $\mathfrak{M}(A ; m, n ; P)$. Estas álgebras também são conhecidas como álgebras de Munn.

O teorema a seguir caracteriza os semigrupos inversos completamente 0-simples:

Teorema 1.3.16[13, Theorem 3.9]. As seguintes condições sobre um semigrupo $S$ com zero são equivalentes:

(i) $S$ é um semigrupo inverso completamente 0-simples;

(ii) $S$ é isomorfo a um semigrupo $S^{0}(G ; m, m ; \mathrm{Id})$ sobre um grupo com zero $G^{0}$ em que Id é a matriz identidade de dimensão $m \times m$.

\footnotetext{
${ }^{3} \mathrm{O}$ resultado continua válido se for assumido que o grupo é periódico em vez de finito, mas o conceito de periodi-
} cidade não será utilizado neste texto. 
Finalmente, o próximo lema mostra como é a álgebra de semigrupo contraída no caso de semigrupos completamente 0 -simples.

Lema 1.3.17[13, Lemma 5.17]. A álgebra de semigrupo contraída $K_{0}[S]$ de $S=S^{0}(G ; m, n, P)$ sobre o corpo $K$ é isomorfa à álgebra $\mathcal{B}=\mathfrak{M}(K G, m, n, P)$.

Para a conveniência do leitor, são lembrados também os seguintes conceitos da teoria de ordem que serão utilizados no Capítulo 3:

Definição 1.3.18. Um conjunto parcialmente ordenado $(P, \leq)$ é chamado de reticulado se para quaisquer $x, y \in P$, existem o supremo e o ínfimo de $\{x, y\}$. Neste caso, denota-se o supremo por $x \vee y$ e o ínfimo por $x \wedge y$.

Observe que, em particular, $(P, \vee)$ e $(P, \wedge)$ são semigrupos comutativos em que todo elemento é idempotente, ou seja, semirreticulados.

Definição 1.3.19. Um reticulado limitado é um reticulado que possui máximo (denotado por 1) e mínimo (denotado por 0).

Definição 1.3.20. Um reticulado completo é um reticulado em que todo subconjunto possui supremo e ínfimo.

\subsection{O Monoide $S(G)$ e Suas $\mathcal{J}$-classes}

A cada grupo $G$, pode-se associar um monoide inverso como em [27, Definition 2.1]:

Definição 1.4.1. O monoide gerado pelos símbolos $\{[g] \mid g \in G\}$ com as relações

(i) $[1][g]=[g][1]=[g]$,

(ii) $\left[g^{-1}\right][g][h]=\left[g^{-1}\right][g h]$.

(iii) $[g][h]\left[h^{-1}\right]=[g h]\left[h^{-1}\right]$,

será denotado por $\mathcal{S}(G){ }^{4}$

Foi demonstrado em [30] que existe um isomorfismo de semigrupos

$$
\mathcal{S}(G) \simeq \tilde{G}^{\mathcal{R}}=\{(A, g)|\{1, g\} \subset A \subset G,| A \mid<\infty\},
$$

sendo que neste conjunto a multiplicação é dada pela regra

$$
(A, g)(B, h)=(A \cup g B, g h) .
$$

Tal isomorfismo é induzido pela aplicação $[g] \mapsto(\{1, g\}, g)$ e deste modo $\mathcal{S}(G)$ é isomorfo à expansão de Birget-Rhodes de $G$ (para mais detalhes, pode-se consultar [30] e [40]). No presente trabalho, $\mathcal{S}(G)$ será identificado com $\tilde{G}^{\mathcal{R}}$, mas isso não deverá causar confusão. Em particular, denotando por $E(S)$ o conjunto dos idempotentes de um semigrupo $S$, nota-se que os idempotentes de $\mathcal{S}(G)$ são dados por

$$
E(\mathcal{S}(G))=\{(A, 1)|1 \in A \subset G,| A \mid<\infty\} .
$$

Para descrever as $\mathcal{J}$-classes de $\mathcal{S}(G)$ será utilizado o seguinte conceito:

Definição 1.4.2. Dados subconjuntos $A$ e $B$ de $G$, ambos contendo 1, define-se:

- $A \preceq B$ se, e somente se, existe algum $x \in G$ tal que $x A \subseteq B$;

- $A \sim B$ se, e somente se, $A \preceq B$ e $B \preceq A$.

\footnotetext{
${ }^{4} \mathrm{Em}$ alguns artigos este monoide é denotado por $E(G)$, mas neste texto a notação $E(S)$ será reservada para indicar o conjunto dos idempotentes de $S$.
} 
O fato a seguir encontra-se em [20, Lemma 2]:

Lema 1.4.3. Para que $(B, b) \in \mathcal{S}(G)(A, a) \mathcal{S}(G)$, ou seja, que $(B, b)$ esteja no ideal gerado por $(A, a)$, é necessário e suficiente que $A \preceq B$.

Com essas notações, [20, Corollary 2] fornece a seguinte descrição para as $\mathcal{J}$-classes de $\mathcal{S}(G)$ :

Lema 1.4.4. A $\mathcal{J}$-classe de cada $(A, a) \in \mathcal{S}(G)$ é o conjunto finito

$$
\mathcal{J}_{A}=\mathcal{J}_{(A, a)}=\{(B, b) \mid B \sim A, 1, b \in B\}=\left\{\left(a^{-1} A, a^{-1} b\right) \mid a, b \in A\right\} .
$$

Em particular, $|A|=|B|$ sempre que $(B, h) \in \mathcal{J}_{(A, a)}$.

Vale ressaltar a seguinte propriedade de $\mathcal{S}(G)$ :

Lema 1.4.5. Para qualquer grupo $G$, o semigrupo $\mathcal{S}(G)$ é completamente semissimples.

Demonstração. Uma vez que toda $\mathcal{J}$-classe $\mathcal{J}_{A}$ possui um idempotente $(A, 1)$, resulta da Observação 1.3.11 que $\mathcal{J}_{A}^{0}$ é 0 -simples e $\operatorname{ker}(\mathcal{S}(G)$ ) é simples (pois a multiplicação nestes semigrupos não é nula). Também segue do Lema 1.4.4 que todas as $\mathcal{J}$-classes de $\mathcal{S}(G)$ são finitas, e como todo fator principal de $\mathcal{S}(G)$ é da forma $\mathcal{J}_{A}^{0}$ (ou simplesmente $\mathcal{J}_{A}$ no caso da $\mathcal{J}$-classe que coincide com $\operatorname{ker}(\mathcal{S}(G))$, conclui-se que eles são finitos e, pelo Lema 1.3.13, completamente (0-)simples.

Lembrando-se que as $\mathcal{J}$-classes de $\mathcal{S}(G)$ podem ser indexadas por subconjuntos de $G$ contendo a unidade (pelo Lema 1.4.4), tem-se o seguinte fato:

Lema 1.4.6[20, Lemma 3]. Seja $\mathcal{J}_{A}$ uma $\mathcal{J}$-classe de $\mathcal{S}(G)$. Então $\mathcal{J}_{A}^{0}$ é um semigrupo inverso completamente 0-simples da forma

$$
S^{0}(\mathrm{St} A ; A / \mathrm{St} A, A / \mathrm{St} A ; \mathrm{Id}),
$$

em que St $A=\{x \in G \mid x A=A\}$ é um subgrupo, $A$ é uma união de classes laterais à direita de St $A$, o conjunto $A / \mathrm{St} A$ é um transversal de tais classes laterais e Id é a matriz identidade de dimensões $\mid A /$ St $A|\times| A /$ St $A \mid$.

\subsection{Representações Parciais Projetivas}

Definição 1.5.1. Diz-se que um semigrupo $S$ com 0 é um $K$-semigrupo se existe uma aplicação $K \times S \rightarrow S$ que satisfaz as seguintes propriedades, para quaisquer $\alpha, \beta \in K$ e $x, y \in S$ :

- $\alpha(\beta x)=(\alpha \beta) x$,

- $\alpha(x y)=(\alpha x) y=x(\alpha y)$,

- $1_{K} x=x$,

- $0_{K} x=0_{S}$.

Um exemplo de $K$-semigrupo é o monoide Mat $_{n} K$ formado pelas matrizes $n \times n$ com entradas em $K$. Esse monoide tem ainda uma outra característica importante: ao multiplicar escalares distintos por uma mesma matriz não-nula, as matrizes resultantes são distintas. Os $K$-semigrupos com essa propriedade recebem um nome especial:

Definição 1.5.2. Um semigrupo $M$ (com zero) é dito $K$-cancelativo se ele é um $K$-semigrupo tal que, para quaisquer $\alpha, \beta \in K$ e $x \in M \backslash\{0\}$, vale

$$
\alpha x=\beta x \Rightarrow \alpha=\beta .
$$

Em um monoide $K$-cancelativo $M$, a congruência $\lambda$ definida por

$$
x \lambda y \Leftrightarrow x=\alpha y, \text { para algum } \alpha \in K^{*}
$$


determina o quociente $\operatorname{Proj} M=M / \lambda$ e a projeção canônica $\xi: M \rightarrow \operatorname{Proj} M$. No caso em que $M=\operatorname{Mat}_{n} K$, o quociente $\operatorname{Proj} M$ é justamente o espaço projetivo PMat $_{n} K$ de matrizes $n \times n$ sobre $K$.

Definição 1.5.3. Um homomorfismo parcial (unital) de um grupo $G$ com valores em um monoide $M$ é uma aplicação $\phi: G \rightarrow M$ que preserva a unidade e satisfaz as seguintes propriedades:

$$
\begin{gathered}
\phi(g) \phi(h) \phi\left(h^{-1}\right)=\phi(g h) \phi\left(h^{-1}\right), \\
\phi\left(g^{-1}\right) \phi(g) \phi(h)=\phi\left(g^{-1}\right) \phi(g h) .
\end{gathered}
$$

para quaisquer $g, h \in G$.

Definição 1.5.4. Seja $M$ um monoide $K$-cancelativo e $G$ um grupo. Uma representação parcial projetiva de $G$ em $M$ é um função $\Gamma: G \rightarrow M$ tal que a composição $\xi \Gamma: G \rightarrow \operatorname{Proj} M$ é um homomorfismo parcial.

Conforme [19, Theorem 3], dada uma representação parcial projetiva $\Gamma: G \rightarrow M$, existe uma única função parcialmente definida $\sigma: G \times G \rightarrow K^{*}$, tal que

$$
\operatorname{dom} \sigma=\{(x, y) \mid \Gamma(x) \Gamma(y) \neq 0\}
$$

e para todo $(x, y) \in \operatorname{dom} \sigma$

$$
\Gamma\left(x^{-1}\right) \Gamma(x) \Gamma(y)=\Gamma\left(x^{-1}\right) \Gamma(x y) \sigma(x, y)
$$

e

$$
\Gamma(x) \Gamma(y) \Gamma\left(y^{-1}\right)=\Gamma(x y) \Gamma\left(y^{-1}\right) \sigma(x, y) .
$$

Por conveniência, define-se $\sigma(x, y)=0$ quando $(x, y) \notin \operatorname{dom} \sigma$ (tornando $\sigma$ totalmente definida), mantendo-se a definição de $\operatorname{dom} \sigma$ como em (1.2), e assume-se (sem perda de generalidade) que $\Gamma(1)=1$.

Definição 1.5.5. A função $\sigma$ associada à representação parcial projetiva $\Gamma$ como no parágrafo anterior é chamada de conjunto fator parcial de $\Gamma$ (ou simplesmente conjunto fator de $G$, subentendendose que está sendo considerada alguma representação parcial projetiva de $G$ ).

Em [19, seção 6] os autores introduziram o monoide $\mathcal{T}$ gerado por símbolos $u, v$ e $t$ com relações

$$
u^{2}=v^{2}=(u v)^{3}=1, t^{2}=t, u t=t, \text { tuvt }=t v u v, t v t=0 .
$$

Pode-se verificar que há uma união disjunta

$$
\mathcal{T}=\mathcal{S} \cup t \mathcal{S} \cup v t \mathcal{S} \cup u v t \mathcal{S} \cup 0
$$

em que $\mathcal{S}=\left\langle u, v \mid u^{2}=v^{2}=(u v)^{3}=1\right\rangle$ é um grupo isomorfo ao grupo simétrico $S_{3}$.

Dado um grupo arbitrário $G$, há uma ação à esquerda de $\mathcal{T}$ em $G \times G$ definida por meio das seguintes transformações:

$$
t(x, y)=(x, 1), \quad u(x, y)=\left(x y, y^{-1}\right) \quad \text { e } \quad v(x, y)=\left(y^{-1}, x^{-1}\right), \text { para quaisquer } x, y \in G .
$$

Segue de (1.3) e (1.5) que $0(x, y)=(1,1)$, para quaisquer $x, y \in G$, e há uma ação de $S_{3}$ em $G \times G$ induzida pela ação de $\mathcal{T}$. Assim, a $S_{3}$-órbita de um par $(x, y) \in G \times G$ é da forma

$$
S_{3}(x, y)=\left\{(x, y),\left(x y, y^{-1}\right),\left(y, y^{-1} x^{-1}\right),\left(y^{-1}, x^{-1}\right),\left(y^{-1} x^{-1}, x\right),\left(x^{-1}, x y\right)\right\}
$$

e contém 1,2,3 ou 6 elementos (ver [21, p. 216], em que a $S_{3}$-órbita que contém $(a, b)$ é denotada por $\left.A_{(a, b)}\right)$. Como em [34], as órbitas com 2 ou 6 elementos são denominadas órbitas efetivas. Assim, 
as órbitas não efetivas têm a forma

$$
\left\{(1, y),\left(y, y^{-1}\right),\left(y^{-1}, 1\right)\right\} \text {, com } y \in G \text {. }
$$

Observação 1.5.6. A importância do monoide $\mathcal{T}$ reside no fato estabelecido em [19, Corollary 7] de que os $\mathcal{T}$-subconjuntos $D$ de $G \times G$, isto é, os elementos de

$$
C(G)=\{D \subseteq G \times G \mid \mathcal{T} D \subseteq D\}=\{D \subseteq G \times G \mid \mathcal{T} D=D\}
$$

são precisamente os dominios dos conjuntos fatores das representações parciais projetivas de $G$ (ver [19, Theorem 5]). Sendo assim, eles formam um semirreticulado em relação à inclusão e à interseção de conjuntos (na verdade, se for considerada também a operação de união de conjuntos, $C(G)$ é um reticulado).

Além dessa caracterização dos domínios como subconjuntos $\mathcal{T}$-invariantes de $G \times G$, observe que de acordo com [19, Corollary 5], os conjuntos fatores $\sigma$ de todas as representações parciais projetivas de $G$ formam um monoide inverso comutativo $\operatorname{pm}(G)$, com relação à multiplicação ponto a ponto.

Definição 1.5.7. O multiplicador de Schur parcial de $G$ é o semigrupo quociente $p M(G)=$ $\operatorname{pm}(G) / \sim$, em que a equivalência $\sim$ é dada por

$$
\sigma \sim \tau \Leftrightarrow \sigma(x, y)=\eta(x) \eta(x y)^{-1} \eta(y) \tau(x, y), x, y \in G
$$

para alguma função $\eta: G \rightarrow K^{*}$.

Por serem semigrupos inversos comutativos, tanto $p m(G)$ quanto $p M(G)$ são semirreticulados de grupos abelianos, conforme o teorema a seguir:

Teorema 1.5.8[19, Theorem 5]. Os semigrupos $\operatorname{pm}(G)$ e $p M(G)$ são semirreticulados de grupos abelianos

$$
p m(G)=\bigcup_{D \in C(G)} p m_{D}(G), \quad p M(G)=\bigcup_{D \in C(G)} p M_{D}(G),
$$

em que $C(G)$ é o semirreticulado dos subconjuntos $\mathcal{T}$-invariantes de $G \times G$ com relação à interseção de conjuntos, $\operatorname{pm}_{D}(G)=\{\sigma \in p m(G) \mid \operatorname{dom} \sigma=D\}$ e $p M_{D}(G)=p m_{D}(G) / \sim$.

Supondo que o corpo $K$ é algebricamente fechado, tem-se ainda a seguinte caracterização dos conjuntos fatores parciais $\sigma$ :

Teorema 1.5.9[21, Theorem 5.6]. Se $\tau$ é um conjunto fator parcial de $G$ com domínio $D$ então existe um conjunto fator parcial $\sigma \sim \tau$, que satisfaz:

$$
\begin{gathered}
\sigma(a, b) \sigma\left(b^{-1}, a^{-1}\right)=1_{K}, \\
\sigma(a, b)=\sigma\left(b^{-1} a^{-1}, a\right)=\sigma\left(b, b^{-1} a^{-1}\right), \\
\sigma(a, 1)=1_{K},
\end{gathered}
$$

para todo $(a, b) \in D$. Reciprocamente, se $\sigma: G \times G \rightarrow K$ é uma função parcialmente definida com $\operatorname{dom} \sigma \in C(G)$ tal que (1.8), (1.9) e (1.10) são satisfeitas para todo $(a, b) \in D$, então $\sigma$ é um conjunto fator parcial de $G$.

Para cada $D \in C(G)$, o subgrupo de $\operatorname{pm}_{D}(G)$ formado por todas as aplicações $\sigma: G \times G \rightarrow K^{*}$ que satisfazem (1.8), (1.9) e (1.10) será denotado por $\mathrm{pm}_{D}^{\prime}(G)$.

Observação 1.5.10. Resulta da demonstração do Teorema 1.5.9 que um conjunto fator $\sigma \in$ $p m_{D}^{\prime}(G)$ fica completamente determinado por seus valores em um conjunto completo de representantes das órbitas efetivas de $D$.

Corolário 1.5.11[21, Corollary 5.8]. Se D é o dominio de um conjunto fator parcial de um grupo arbitrário $G$, então: 
(i) Todo conjunto fator parcial de $\mathrm{pm}_{D}(G)$ é equivalente a um elemento de $\mathrm{pm}_{D}^{\prime}(G)$.

(ii) O núcleo $N_{D}=\left\{\sigma \in p m_{D}^{\prime}(G) \mid \sigma \sim 1\right\}$ do epimorfismo natural de $p m_{D}^{\prime}(G) \rightarrow p M_{D}(G)$ consiste dos $\sigma: G \times G \rightarrow K$ para os quais existe $\rho: G \times G \rightarrow K^{*}$ satisfazendo as seguintes condiçôes:

$$
\rho(1)=1_{K},
$$

$$
\begin{gathered}
\rho(a) \rho\left(a^{-1}\right)=1, \text { para todo } a \in G \text { tal que }(a, 1) \in D, \\
\sigma(a, b)= \begin{cases}\rho(a) \rho(b) \rho(a b)^{-1}, & \text { se }(a, b) \in D, \\
0, & \text { se }(a, b) \notin D .\end{cases}
\end{gathered}
$$

(iii) Sejam $s=s(G, D)$ a cardinalidade do conjunto de $S_{3}$-órbitas efetivas de $D$ e $\left\{\left(a_{i}, b_{i}\right)\right\}_{1 \leq i \leq s}$ um conjunto completo de representantes de tais órbitas. Então a aplicação

$$
\phi:\left(K^{*}\right)^{s} \ni x \mapsto \sigma_{x} \in p m_{D}^{\prime}(G),
$$

em que $x=\left(x_{i}\right)_{1 \leq i \leq s}$ e $\sigma_{x}\left(a_{i}, b_{i}\right)=x_{i}$, é um isomorfismo de grupos multiplicativos.

(iv) Para todo dominio $Y \in C(G)$ tal que $Y \supseteq D$, tem-se um epimorfismo $\psi_{D}^{Y}: p M_{Y}(G) \rightarrow$ $p M_{D}(G)$. Em particular, $p M_{D}(G)$ é uma imagem epimorfa da componente total $p M_{G \times G}(G)$.

Para finalizar este capítulo, definindo

$$
L_{D}=\left\{x \in\left(K^{*}\right)^{s} \mid \sigma_{x} \sim 1\right\}=\left\{x \in\left(K^{*}\right)^{s} \mid \sigma_{x} \in N_{D}\right\}
$$

tem-se o seguinte:

Teorema 1.5.12[21, Theorem 5.9]. Se $D \in C(G)$ e $s=s(G, D)$ é a cardinalidade do conjunto de $S_{3}$-órbitas efetivas de $D$, então há os seguintes isomorfismos:

$$
p M_{D}(G) \simeq p m_{D}^{\prime}(G) / N_{D} \simeq\left(K^{*}\right)^{s} / L_{D} .
$$

Em particular, será utilizado o epimorfismo

$$
\psi:\left(K^{*}\right)^{s} \ni x \mapsto \operatorname{cls}\left(\sigma_{x}\right) \in p M_{D}(G),
$$

para o qual $\operatorname{ker}(\psi)=L_{D}$. Aqui, $\operatorname{cls}\left(\sigma_{x}\right)$ indica a classe de equivalência de $\sigma_{x}$ em $p M_{D}(G)$. 


\section{Capítulo 2}

\section{Revisitando Alguns Fatos Conhecidos}

Neste capítulo, serão discutidos alguns resultados que foram apresentados originalmente nos artigos $[16,24,20]$. Na primeira seção, será dada uma demonstração alternativa para a decomposição da álgebra parcial de grupo em soma de álgebras matriciais sobre álgebras de subgrupos. Em seguida, será demonstrada uma proposição que completa uma lacuna existente na demonstração de [24, Theorem 2.2], sobre as representações parciais de grau finito de grupos. Para encerrar o capítulo, será discutida a interação entre ações parciais e representações parciais em álgebras.

\subsection{Decomposição da Álgebra Parcial de Grupo}

A álgebra parcial de grupo $K_{\text {par }}(G)$ introduzida na Definição 1.2.2 é justamente a álgebra de semigrupo de $\mathcal{S}(G)$ sobre o corpo $K$. Se $G$ é um grupo finito, e $\mathcal{C}$ denota um conjunto completo de representantes das classes de conjugação dos subgrupos de $G$ então, conforme [23, Theorem 2.1], há um isomorfismo

$$
\psi: K_{\mathrm{par}}(G) \rightarrow \bigoplus_{\substack{H \in \mathcal{C} \\ 1 \leq m \leq(G: H)}} c_{m}(H) M_{m}(K H),
$$

em que $c_{m}(H) M_{m}(K H)$ significa a soma direta de $c_{m}(H)$ cópias de $M_{m}(K H)$. O leitor interessado deve consultar [23] para obter detalhes sobre as multiplicidades $c_{m}(H)$. Por conveniência, simplificaremos a notação anterior para $\psi: K_{\text {par }}(G) \rightarrow \oplus M_{m}(K H)$, ficando implícito que pode ocorrer a repetição de somandos com o mesmo $m$ e o mesmo $H$.

Conforme sugerido em [20, Remark 1], também é possível estabelecer o isomorfismo dado em (2.1) por meio da teoria de álgebras de semigrupos para semigrupos inversos. Isso será feito no Teorema 2.1.2 e o Exemplo 2.1.4 mostrará como é essa abordagem no caso do grupo cíclico de ordem 3. Para isso, é bom ter em mente o seguinte resultado:

Lema 2.1.1[20, Remark 1]. Para todo fator principal $\mathcal{J}_{A}^{0}$ de $\mathcal{S}(G)$, tem-se

$$
K_{0} \mathcal{J}_{A}^{0} \simeq K_{0} S^{0}(H ; m, m ; \mathrm{Id}) \simeq M_{m}(K H),
$$

em que $H=\operatorname{St} A=\{g \in G \mid g A=A\}$ e $m=|A| /|\operatorname{St} A|$.

Demonstração. De acordo com o Lema 1.4.6, cada $\mathcal{J}_{A}^{0}$ é um semigrupo inverso completamente 0 -simples da forma $S^{0}(\mathrm{St} A ; A / \mathrm{St} A, A / \mathrm{St} A ; \mathrm{Id})$, em que $A / \mathrm{St} A$ é um conjunto completo de representantes das classes laterais à direita de St $A$ que estão contidas em $A$. Além disso, o Lema 1.3 .17 garante que $K_{0} S^{0}(G ; m, n ; P) \simeq \mathfrak{M}(K G ; m, n ; P)$, para qualquer grupo $G$. Em particular, se $G=$ St $A$, $m=n=|A| / \mid$ St $A \mid$ e $P=\mathrm{Id}$, tem-se o isomorfismo desejado, a saber, $\phi: K_{0} S^{0}(H ; m, m ; \mathrm{Id}) \rightarrow$ $M_{m}(K H)$ definido por

$$
\phi\left(\sum_{i=1}^{m} \sum_{j=1}^{m} \sum_{g \in H} c_{i, j, g}(i, g, j)\right)=\sum_{i=1}^{m} \sum_{j=1}^{m}\left(\sum_{g \in H} c_{i, j, g} g\right) e_{i, j} .
$$


onde $e_{i, j}$ denota a matriz com a unidade na posição $(i, j)$ e zero nas demais posições.

O isomorfismo (2.3) abaixo é um caso particular de um fato conhecido na teoria de álgebras de semigrupos. O objetivo aqui é mostrar que sua prova pode ser usada para obter a decomposição (2.4) de $K_{\mathrm{par}}(G)$, de forma alternativa à demonstração feita em [16].

Teorema 2.1.2 Ver [35, Chapter 5, Corollary 27]. Se $S$ é um semigrupo inverso finito, então

$$
K_{0}[S] \simeq \bigoplus_{i \in \mathcal{I}} M_{m_{i}}\left(K H_{i}\right)
$$

para certos subgrupos $H_{i} \subseteq S$ e inteiros $m_{i} \geq 1$, onde cada $M_{m_{i}}\left(K H_{i}\right)$ é uma álgebra de semigrupo contraída de um fator principal não-nulo de $S$. Em particular [16], para um grupo G finito,

$$
K_{\mathrm{par}}(G) \simeq \bigoplus_{i \in \mathcal{I}} M_{m_{i}}\left(K H_{i}\right)
$$

e neste caso $H_{i}$ é um subgrupo de $G$, para todo $i \in \mathcal{I}$. Além disso, $G \simeq \operatorname{ker}(\mathcal{S}(G))$.

Demonstração. Evidentemente, pode-se assumir que $S \neq 0$. Seja $I$ um ideal 0-minimal de $S$.

Se $S$ contém 0, então $I$ é um semigrupo inverso completamente 0 -simples e, consequentemente, por [13, Theorem 3.9], I é isomorfo ao semigrupo $S^{0}(G ; n, n ; \mathrm{Id})$, para algum grupo finito $G$ e algum inteiro positivo $n$. O isomorfismo acima (2.2) mostra que $K_{0}[I] \simeq K_{0}\left[S^{0}(G ; n, n, \mathrm{Id})\right] \simeq M_{n}(K G)$. Em particular, $K_{0}[I]$ tem unidade.

Se $S$ não possui zero, então $I$ é um semigrupo inverso completamente simples. Como tal, $I$ é na verdade um grupo $G$ ( $I^{0}$ é completamente 0 -simples, de modo que $I \simeq S^{0}(G ; n, n ; \mathrm{Id}) \backslash\{0\}$ e este último só não possui divisores de zero quando $n=1$ ). Neste caso, $K I=K_{0}[I]$ é uma álgebra de grupo, e tem unidade. Note que em ambas as situações, $K_{0}[I]$ é a álgebra de semigrupo contraída de um fator principal não-nulo de $S$.

Tendo isso em mente, a decomposição será obtida por indução no número de $\mathcal{J}$-classes não-nulas de $S$. Se $S$ possui apenas uma $\mathcal{J}$-classe não-nula, então $S=I$, e não é preciso fazer mais nada.

Assuma que o número de $\mathcal{J}$-classes não-nulas de $S$ é $k>1$ e suponha que a decomposição (2.3) seja válida para todo semigrupo inverso em que a quantidade de $\mathcal{J}$-classes não-nulas é no máximo $k-1$. Como $K_{0}[I]$ é uma álgebra com unidade, pode-se aplicar a Observação 1.3.5 para obter

$$
K_{0}[S] \simeq K_{0}[I] \oplus K_{0}[S / I]
$$

A projeção canônica de $S$ sobre $S / I$ induz uma correspondência biunívoca entre os fatores principais de $S \backslash I$ e os fatores principais não-nulos de $S / I$, segundo a qual fatores principais associados são isomorfos. Consequentemente, a quantidade de $\mathcal{J}$-classes não-nulas do semigrupo inverso $S / I$ é menor do que $k$ e, pela hipótese de indução,

$$
K_{0}[S / I] \simeq \bigoplus_{i \in \mathcal{I}} M_{m_{i}}\left(K H_{i}\right)
$$

Assim, a decomposição resulta de (2.5).

De modo a obter (2.4) observe o seguinte:

- Conforme o Lema 1.4.6, quando a $\mathcal{J}$-classe $\mathcal{J}_{A}=\mathcal{J}_{(A, 1)} \neq \operatorname{ker}(\mathcal{S}(G))$, o fator principal é $\mathcal{J}_{A}^{0} \simeq S^{0}(\mathrm{St} A ; A / \mathrm{St} A, A / \mathrm{St} A ; \mathrm{Id})$, e $\mathrm{St} A$ é um subgrupo de $G$;

- $\operatorname{ker}(\mathcal{S}(G))=N=\{(G, g) \mid g \in G\} \simeq G$. Para cada $(B, h)$

$$
(G, g)(B, h)=(G \cup g B, g h)=(G, g h) \in N,
$$

e de forma análoga, $(B, h)(G, g)=(G, h g) \in N$. Disto conclui-se que a aplicação $g \mapsto(G, g) \in$ $N$ fornece um isomorfismo entre o grupo $G$ e $\operatorname{ker}(\mathcal{S}(G))$. 
Seja $S$ um monoide inverso finito e $C=\left\{\mathcal{J}_{1}, \ldots, \mathcal{J}_{k}\right\}$ o conjunto das $\mathcal{J}$-classes não-nulas de $S$, indexadas de tal modo que em relação à ordem parcial definida por (1.1) ocorra o seguinte:

- A $\mathcal{J}$-classe $\mathcal{J}_{1}$ é minimal em $C$;

- A $\mathcal{J}$-classe $\mathcal{J}_{i}$ é minimal em $C \backslash\left\{\mathcal{J}_{1}, \ldots, \mathcal{J}_{i-1}\right\}$, para cada $i>1$.

Aqui, a minimalidade é em relação à ordem parcial induzida pela inclusão dos ideais principais correspondentes. Note que se $S$ não possui zero, $\mathcal{J}_{1}=\operatorname{ker}(S)$. Caso contrário, defina $\mathcal{J}_{0}=\operatorname{ker}(S)=$ $\{0\}$. Seja $m_{i}$ a quantidade de idempotentes em $\mathcal{J}_{i}$ e $e_{i} \in K_{0} S$ a sua soma. Com estas notações, tem-se o seguinte:

Proposição 2.1.3. Se S é um monoide inverso finito não-nulo, então os elementos

$$
\begin{aligned}
f_{1} & =e_{1}, \\
f_{2} & =\left(1-e_{1}\right) e_{2}, \\
f_{3} & =\left(1-e_{1}\right)\left(1-e_{2}\right) e_{3}, \\
& \ldots \\
f_{k-1} & =\left(1-e_{1}\right) \ldots\left(1-e_{k-2}\right) e_{k-1} e \\
f_{k} & =\left(1-e_{1}\right) \ldots\left(1-e_{k-2}\right)\left(1-e_{k-1}\right)
\end{aligned}
$$

são idempotentes centrais ortogonais de $K_{0} S$ e

$$
K_{0} S=\bigoplus_{i=1}^{k}\left(K_{0} S\right) f_{i} \simeq \bigoplus_{i=1}^{k} M_{m_{i}}\left(K H_{i}\right) .
$$

Em particular, para $K_{\mathrm{par}} G$ o elemento $e_{i}$ correspondente a $\mathcal{J}_{A_{i}}$ é dado por

$$
e_{i}=\sum_{a \in A_{i} / \mathrm{St} A_{i}}\left(a^{-1} A_{i}, 1\right)
$$

em que $A / \mathrm{St} A \subseteq A$ denota um conjunto de representantes das classes laterais à direita de $\mathrm{St} A$.

Demonstração. De acordo com o Teorema 1.3.16, cada um dos semigrupos inversos completamente 0 -simples $\mathcal{J}_{i}^{0}$ é isomorfo a um semigrupo de Rees do tipo $S^{0}\left(H_{i} ; m_{i}, m_{i} ; \mathrm{Id}\right)$ e, segundo [13, Lemma 5.17],

$$
K_{0} S^{0}\left(H_{i} ; m_{i}, m_{i} ; \mathrm{Id}\right) \simeq \mathfrak{M}\left(K H_{i} ; m_{i}, m_{i} ; \mathrm{Id}\right)=M_{m_{i}}\left(K H_{i}\right) .
$$

Dado um isomorfismo $\phi_{i}: K_{0} \mathcal{J}_{i}^{0} \rightarrow M_{m_{i}}\left(K H_{i}\right)$, a unidade de $K_{0} \mathcal{J}_{i}^{0}$ é

$$
\phi_{i}^{-1}\left(\operatorname{Id}_{m_{i}}\right)=\sum_{f \in E(S) \cap \mathcal{J}_{i}} f=e_{i} .
$$

O resultado será obtido por indução sobre a quantidade de $\mathcal{J}$-classes não-nulas de $S$.

Suponha que há apenas uma $\mathcal{J}$-classe não-nula. Então a unidade de $S$ está em $\mathcal{J}_{1}$ e coincide com a unidade 1 da álgebra $K_{0} S=K_{0} \mathcal{J}_{1}^{0}$. Além disso, essa unidade coincide com $e_{1}$, pois o semigrupo de Rees $\mathcal{J}_{1}^{0} \simeq S^{0}\left(H_{1} ; m_{1}, m_{1} ; \mathrm{Id}\right)$ só tem unidade se $m_{1}=1$. Consequentemente, a decomposição (2.6) reduz-se a igualdade trivial $K_{0} S=K_{0} S \oplus 0=K_{0} S e_{1} \oplus K_{0} S\left(1-e_{1}\right)$.

Suponha que o monoide $S$ possui $k>1 \mathcal{J}$-classes não-nulas. Assuma que a decomposição (2.6) seja válida para todo monoide inverso finito com no máximo $k-1 \mathcal{J}$-classes. Seja $I=\mathcal{J}_{1} \cup \mathcal{J}_{0} \simeq \mathcal{J}_{1}^{0}$ se $S$ possui zero, e $I=\mathcal{J}_{1}$ caso contrário. Em ambos os casos, $I$ é um ideal de $S$ e tem-se:

$$
K_{0} S=K_{0} S e_{1} \oplus K_{0} S\left(1-e_{1}\right) \simeq K_{0} I \oplus K_{0}[S / I] .
$$


Observe que há um isomorfismo $\phi: K_{0}[S / I] \rightarrow K_{0} S\left(1-e_{1}\right)$, segundo o qual cada elemento $x \in S \backslash I$, visto como um elemento de $S / I$, é levado em $x\left(1-e_{1}\right) \in K_{0} S\left(1-e_{1}\right)$. Uma vez que a quantidade de $\mathcal{J}$-classes não-nulas no semigrupo quociente $S / I$ é exatamente $k-1$, pode-se aplicar a indução e obter:

$$
\begin{aligned}
K_{0}[S / I] & =K_{0}[S / I] e_{2} \\
& \oplus K_{0}[S / I]\left(1-e_{2}\right) e_{3} \\
& \oplus K_{0}[S / I]\left(1-e_{2}\right)\left(1-e_{3}\right) e_{4} \\
& \oplus \ldots \\
& \oplus K_{0}[S / I]\left(1-e_{2}\right) \ldots\left(1-e_{k-2}\right) e_{k-1} \\
& \oplus K_{0}[S / I]\left(1-e_{2}\right) \ldots\left(1-e_{k-2}\right)\left(1-e_{k-1}\right) .
\end{aligned}
$$

Aplicando-se o isomorfismo $\phi$ a cada somando direto, obtém-se:

$$
\begin{aligned}
K_{0} S\left(1-e_{1}\right) & =K_{0} S\left(1-e_{1}\right) e_{2} \\
& \oplus K_{0} S\left(1-e_{1}\right)\left(1-e_{2}\right) e_{3} \\
& \oplus K_{0} S\left(1-e_{1}\right)\left(1-e_{2}\right)\left(1-e_{3}\right) e_{4} \\
& \oplus \ldots \\
& \oplus K_{0} S\left(1-e_{1}\right)\left(1-e_{2}\right) \ldots\left(1-e_{k-2}\right) e_{k-1} \\
& \oplus K_{0} S\left(1-e_{1}\right)\left(1-e_{2}\right) \ldots\left(1-e_{k-2}\right)\left(1-e_{k-1}\right) .
\end{aligned}
$$

Disso resulta a decomposição (2.6), pois sendo $e_{1}$ um idempotente central, $1-e_{1}$ também o será, e um produto de idempotentes centrais é um idempotente central. No caso de $K_{\text {par }}(G)$, a fórmula (2.7) para cada $e_{i}$ é obtida por meio do Lema 1.4.4, segundo o qual a $\mathcal{J}$-classe $\mathcal{J}_{A_{i}}$ é formada pelos elementos da forma $\left(a^{-1} A_{i}, a^{-1} b\right)$, em que $a, b \in A_{i}$. Em particular, os idempotentes de $\mathcal{J}_{A_{i}}$ são aqueles elementos do tipo $\left(a^{-1} A_{i}, 1\right)$, com $a \in A_{i}$, e para que cada idempotente apareça apenas uma vez na definição de $e_{i}$, considera-se apenas um elemento $a \in A_{i}$ em cada classe lateral à direita de $\mathrm{St} A_{i}$, formando-se assim o conjunto $A_{i} / \mathrm{St} A_{i}$ com estes representantes.

Para finalizar esta seção, será calculado $K_{\text {par }} C_{3}$.

Exemplo 2.1.4. Se $C_{3}=\left\langle a \mid a^{3}=1\right\rangle=\left\{1, a, a^{2}\right\}$ então há 8 elementos em $\mathcal{S}\left(C_{3}\right)$, e a sua decomposição em $\mathcal{J}$-classes é

$\mathcal{S}\left(C_{3}\right) \simeq\{(\{1\}, 1)\} \cup\left\{(\{1, a\}, 1),(\{1, a\}, a),\left(\left\{1, a^{2}\right\}, a^{2}\right),\left(\left\{1, a^{2}\right\}, 1\right)\right\} \cup\left\{\left(C_{3}, 1\right),\left(C_{3}, a\right),\left(C_{3}, a^{2}\right)\right\}$, o que implica que $\mathcal{S}\left(C_{3}\right)$ possui três fatores principais:

- $\mathcal{J}_{(\{1\}, 1)}^{0} \simeq S^{0}(\{1\} ; 1,1 ; \mathrm{Id})$,

- $\mathcal{J}_{(\{1, a\}, 1)}^{0} \simeq S^{0}(\{1\} ; 2,2 ; \mathrm{Id}) \mathrm{e}$

- $\mathcal{J}_{C_{3}}=J_{\left(C_{3}, 1\right)}=\operatorname{ker}\left(\mathcal{S}\left(C_{3}\right)\right) \simeq S^{0}\left(C_{3} ; 1,1 ; \mathrm{Id}\right) \simeq C_{3}$.

As $\mathcal{J}$-classes estão ordenadas como na Figura 2.1.

Uma vez que $K C_{3}$ é uma álgebra com unidade, pode-se aplicar o Lema 1.3.4 ao ideal não-nulo minimal $J_{\left(C_{3}, 1\right)}=\mathcal{J}_{C_{3}}$, que é o núcleo de $\mathcal{S}\left(C_{3}\right)$, para obter

$$
K_{\mathrm{par}}\left(C_{3}\right) \simeq K C_{3} \oplus K_{0}\left[\mathcal{S}\left(C_{3}\right) / J_{\left(C_{3}, 1\right)}\right]
$$

Como o ideal $J_{(\{1, a\}, 1)}=\mathcal{J}_{(\{1, a\}, 1)} \cup J_{\left(C_{3}, 1\right)}$, resulta que $J_{(\{1, a\}, 1)} / J_{\left(C_{3}, 1\right)} \simeq \mathcal{J}_{(\{1, a\}, 1)}^{0}$ é um ideal não-nulo minimal de $\mathcal{S}\left(C_{3}\right) / J_{\left(C_{3}, 1\right)}$. Além disso, a álgebra de semigrupo contraída deste ideal tem unidade, pois

$$
K_{0} \mathcal{J}_{(\{1, a\}, 1)}^{0} \simeq K_{0} S^{0}(\{1\} ; 2,2 ; \mathrm{Id}) \simeq M_{2}(K) .
$$


Figura 2.1: Ordenação das $\mathcal{J}$-classes de $\mathcal{S}\left(C_{3}\right)$.

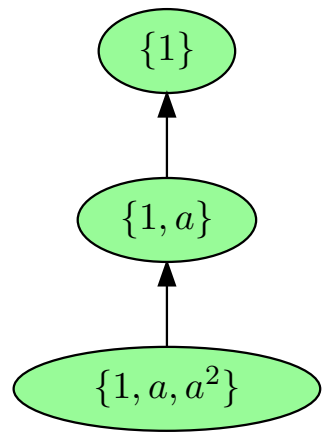

Assim, pode-se aplicar a Observação 1.3.5 ao ideal $J_{(\{1, a\}, 1)} / J_{\left(C_{3}, 1\right)}$, para obter

$$
K_{\mathrm{par}}\left(C_{3}\right) \simeq K C_{3} \oplus K_{0} \mathcal{J}_{(\{1, a\}, 1)}^{0} \oplus K_{0}\left[\left(\mathcal{S}\left(C_{3}\right) / J_{\left(C_{3}, 1\right)}\right) / \mathcal{J}_{(\{1, a\}, 1)}^{0}\right]
$$

$\operatorname{Mas}\left[\left(\mathcal{S}\left(C_{3}\right) / J_{\left(C_{3}, 1\right)}\right) / \mathcal{J}_{(\{1, a\}, 1)}^{0}\right] \simeq \mathcal{S}\left(C_{3}\right) / J_{(\{1, a\}, 1)}$, que é isomorfo ao grupo trivial com um zero adicionado, e portanto

$$
K_{\mathrm{par}}\left(C_{3}\right) \simeq K \oplus M_{2}(K) \oplus K C_{3},
$$

o que está de acordo com a decomposição que foi obtida em [16].

Explicitamente, tem-se o isomorfismo $\phi: K_{\text {par }}\left(C_{3}\right) \rightarrow K \oplus M_{2}(K) \oplus K C_{3}$, dado por

$$
\begin{aligned}
x=[( & \left.\{1\}, 1)-\left(C_{3}, 1\right)\right]\left[(\{1\}, 1)-\left((\{1, a\}, 1)+\left(\left\{1, a^{2}\right\}, 1\right)\right)\right] x \\
& +\left[(\{1\}, 1)-\left(C_{3}, 1\right)\right]\left[(\{1, a\}, 1)+\left(\left\{1, a^{2}\right\}, 1\right)\right] x \\
& +\left(C_{3}, 1\right) x \\
=[ & \left.(\{1\}, 1)-(\{1, a\}, 1)-\left(\left\{1, a^{2}\right\}, 1\right)+\left(C_{3}, 1\right)\right] x \\
& +\left[(\{1, a\}, 1)+\left(\left\{1, a^{2}\right\}, 1\right)-2\left(C_{3}, 1\right)\right] x \\
& +\left(C_{3}, 1\right) x .
\end{aligned}
$$

isto é, se $x=a_{1}(\{1\}, 1)+a_{2}(\{1, a\}, 1)+a_{3}(\{1, a\}, a)+a_{4}\left(\left\{1, a^{2}\right\}, 1\right)+a_{5}\left(\left\{1, a^{2}\right\}, a^{2}\right)+a_{6}\left(C_{3}, 1\right)+$ $a_{7}\left(C_{3}, 1\right)+a_{8}\left(C_{3}, 1\right)$, então:

$$
\begin{aligned}
x \mapsto a_{1} & {\left[(\{1\}, 1)-(\{1, a\}, 1)-\left(\left\{1, a^{2}\right\}, 1\right)+\left(C_{3}, 1\right)\right] } \\
+ & {\left[a_{1}+a_{2}\right](\{1, a\}, 1)+a_{3}(\{1, a\}, a)+\left[a_{1}+a_{4}\right]\left(\left\{1, a^{2}\right\}, 1\right)+a_{5}\left(\left\{1, a^{2}\right\}, a^{2}\right) } \\
+ & {\left[a_{1}+a_{2}+a_{4}+a_{6}\right]\left(C_{3}, 1\right)+\left[a_{3}+a_{7}\right]\left(C_{3}, a\right)+\left[a_{5}+a_{8}\right]\left(C_{3}, a^{2}\right) . }
\end{aligned}
$$

\subsection{Representações Parciais de Grau Finito}

Dado um grupo $G$ arbitrário, define-se em [24, p. 311] o grupoide $\Gamma(G)$ como uma categoria pequena em que os elementos (morfismos) são os pares $(A, g)$ tais que $\left\{1, g^{-1}\right\} \subseteq A \subseteq G$, e os vértices são os pares $(A, 1)$ nas mesmas condições, os quais serão denotados simplesmente por $A$. Dados elementos $(A, g)$ e $(B, h)$ de $\Gamma(G)$, o seu produto só está definido se $A=h B$. Neste caso,

$$
(h B, g)(B, h)=(B, g h) .
$$

O grupoide $\Gamma(G)$ pode ser visto como um grafo orientado cujos vértices são rotulados pelos objetos de $\Gamma(G)$, e no qual um morfismo $(A, g)$ em $\Gamma(G)$ corresponde a uma aresta $A \longrightarrow g A$.

Dado um objeto (vértice) $A$ de $\Gamma(G)$, considera-se a componente conexa de $\Gamma(G)$ que contém $A$ e denota-se por $\mathcal{V}_{A}$ o conjunto de seus vértices. Observa-se que o estabilizador $H=\operatorname{St} A=\{g \in$ 
$G \mid g A=A\}$ é um subconjunto de $A$, e $A$ é uma união de classes laterais à direita de $H$, isto é, $A=\bigcup_{i \in \mathcal{I}} H g_{i}$ para algum conjunto de índices $\mathcal{I}$ e algum transversal $\left\{g_{i} \mid i \in \mathcal{I}\right\} \subseteq A$ contendo $1_{G}$. Além disso, a cardinalidade $\left|\mathcal{V}_{A}\right|$ de $\mathcal{V}_{A}$ é igual a $|\mathcal{I}|$, e $\mathcal{V}_{A}$ consiste de vértices da forma $g_{i}^{-1} A$, com $i \in \mathcal{I}$.

A Figura 2.2 mostra como é este grupoide no caso em que $G=C_{3}=\left\langle a \mid a^{3}=1\right\rangle$.

Figura 2.2: Vértices e arestas de $\Gamma\left(C_{3}\right)$.
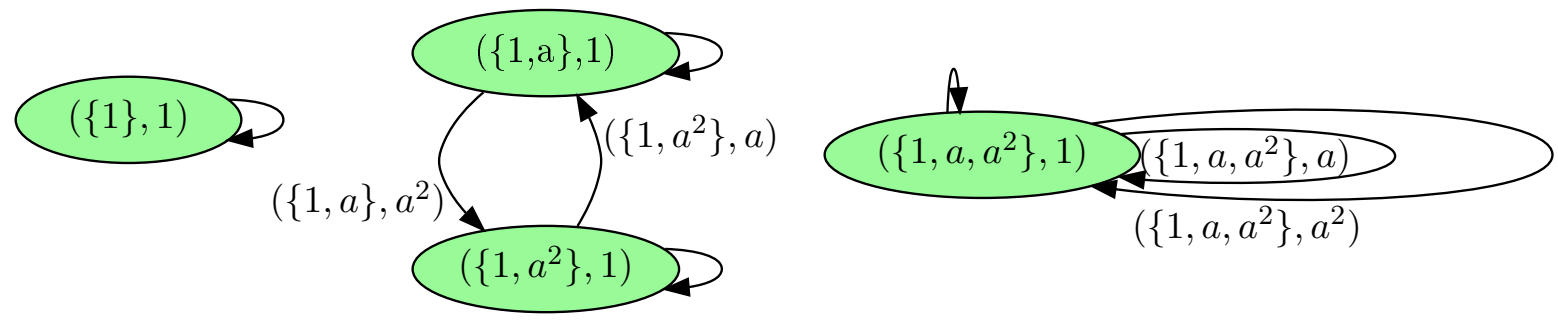

Em [16, p. 9], o grupoide $\Gamma(G)$ foi definido apenas para grupos finitos. Para esclarecer a sua relação com o semigrupo $\mathcal{S}(G)$ no caso de grupos arbitrários, considere o subgrupoide $\Gamma_{\text {fin }}(G)$ de $\Gamma(G)$ que consiste de todos os pares $(A, g)$ tais que $|A|<\infty$.

Conforme [31, Proposition 4], o semigrupo inverso $\mathcal{S}(G)$ pode ser visto como um grupoide $\mathcal{G}(\mathcal{S}(G))$ em relação ao seu produto restrito, que é definido por:

$$
s \cdot t= \begin{cases}s t, & \text { se } s^{-1} s=t t^{-1} \\ \nexists, & \text { caso contrário }\end{cases}
$$

para todo $s, t \in \mathcal{S}(G)$. Utiliza-se a notação $d(s)=s^{-1} s$ para indicar a origem da aresta $s$ e $r(s)=s s^{-1}$ para indicar sua imagem. $\operatorname{Em} \mathcal{S}(G)$, com a notação de Birget-Rhodes, tem-se $(A, g)^{-1}=$ $\left(g^{-1} A, g^{-1}\right)$, então

- $d(A, g)=\left(g^{-1} A, g^{-1}\right)(A, g)=\left(g^{-1} A, 1\right)$,

- $r(A, g)=(A, g)\left(g^{-1} A, g^{-1}\right)=(A, 1)$.

e o produto restrito $(A, g) \cdot(B, h)$ está definido se, e somente se, $\left(g^{-1} A, 1\right)=(B, 1)$, o que equivale a $A=g B$. Para evitar ambiguidade entre $\mathcal{G}(\mathcal{S}(G))$ e $\Gamma(G)$, um elemento $(A, g) \in \Gamma(G)$ será denotado por $\langle A, g\rangle$.

Observação 2.2.1. Se $G$ é um grupo, a aplicação $\phi: \mathcal{G}(\mathcal{S}(G)) \rightarrow \Gamma_{\text {fin }}(G)$, dada por

$$
\phi(A, g)=\left\langle g^{-1} A, g\right\rangle,
$$

é um isomorfismo de grupoides. De fato, é claro que $\phi$ é uma função injetora. Além disso, todo $\langle B, h\rangle$ pertence à imagem de $\phi$, pois como $B$ é finito, $(h B, h) \in \mathcal{S}(G)$ e $\phi(h B, h)=\langle B, h\rangle$. Se $(A, g) \cdot(B, h)$ está definido, então $A=g B$ e

$$
\phi((g B, g) \cdot(B, h))=\phi(g B, g h)=\left\langle(g h)^{-1} g B, g h\right\rangle=\left\langle h^{-1} B, g h\right\rangle .
$$

Por outro lado,

$$
\phi(g B, g) \phi(B, h)=\left\langle g^{-1}(g B), g\right\rangle\left\langle h^{-1} B, h\right\rangle=\left\langle h\left(h^{-1} B\right), g\right\rangle\left\langle h^{-1} B, h\right\rangle=\left\langle h^{-1} B, g h\right\rangle .
$$

Observe ainda que, para todo semigrupo inverso $S$, se $s, t \in S$ são tais que $\exists s \cdot t$, isto é, se $s^{-1} s=t t^{-1}$, então

$$
S^{1} t S^{1}=S^{1} t t^{-1} t S^{1}=S^{1} s^{-1} s t S^{1} \subseteq S^{1} s S^{1}=S^{1} s s^{-1} s S^{1}=S^{1} s t t^{-1} S^{1} \subseteq S^{1} t S^{1} .
$$


Logo, quando o produto restrito $s \cdot t$ está definido, estes elementos pertencem à mesma $\mathcal{J}$-classe. Por transitividade, os elementos da componente conexa de $s$ no grupoide $\mathcal{G}(S)$ estão todos na $\mathcal{J}$-classe de $s$ no semigrupo inverso $S$.

Definição 2.2.2. Dada uma categoria pequena $\mathcal{C}$ e um corpo $K$, a álgebra de categoria $K \mathcal{C}$ é definida tomando-se o $K$-espaço vetorial cuja base é formada pelos morfismos de $\mathcal{C}$ e definindo-se a multiplicação da seguinte forma:

$$
\gamma_{1} \cdot \gamma_{2}= \begin{cases}\gamma_{1} \gamma_{2}, & \text { se existe a composição } \gamma_{1} \gamma_{2} \in \mathcal{C}, \\ 0, & \text { caso contrário. }\end{cases}
$$

Dada uma componente conexa $\Delta$ de $\mathcal{C}=\Gamma(G)$ (isto é, um subgrupoide maximal conexo por morfismos) com um número finito de vértices, define-se a aplicação $\lambda_{\Delta}: G \rightarrow K \Delta$ por:

$$
\lambda_{\Delta}(g)= \begin{cases}\sum_{A \in \mathcal{V}_{\Delta}}(A, g), & \text { se existe } A \in \mathcal{V}_{\Delta} \operatorname{com} g^{-1} \in A, \\ A \ni g^{-1} & \text { caso contrário. }\end{cases}
$$

Em [24, Theorem 2.2] foi demonstrado o seguinte resultado, que descreve a estrutura das representações parciais de $G$ de grau finito:

Teorema 2.2.3. Seja $G$ um grupo. Para cada componente conexa $\Delta$ de $\Gamma(G)$ com um número finito de vértices, a aplicação $\lambda_{\Delta}: G \rightarrow K \Delta$ é uma representação parcial de $G$ em $K \Delta$. Além disso:

(i) Para toda K-representação $\varphi: K \Delta \rightarrow E n d(V)$ de grau finito irredutivel (respectivamente, indecomponivel), $\varphi \circ \lambda_{\Delta}$ é uma representação parcial irredutivel (respectivamente, indecomponível) de $G$.

(ii) Reciprocamente, para toda K-representação parcial de grau finito irredutivel (respectivamente, indecomponivel) $\pi: G \rightarrow \operatorname{End}(V)$, existe uma única componente conexa $\Delta$ de $\Gamma(G)$ com um número finito de vértices e uma única representação $\tilde{\pi}: K \Delta \rightarrow \operatorname{End}(V)$ tal que $\tilde{\pi} \circ \lambda_{\Delta}=\pi$.

No entanto, a prova deste teorema utilizou-se de um fato que para ser justificado no caso de grupos infinitos exigia um argumento um pouco mais elaborado do que aquele apresentado no artigo. A Proposição 2.2.4 complementa a demonstração do Teorema 2.2.3, preenchendo esta lacuna que havia em sua demonstração (ver [24, p. 314-315]).

No que segue, se $(A, g) \in \Gamma(G)$, então $A$ será denominado o conjunto suporte de $(A, g)$ e também será identificado com o vértice $(A, 1)$.

Proposição 2.2.4. Com a notação do Teorema 2.2.3, seja $\Delta$ uma componente conexa de $\Gamma(G)$ cujo número de vértices, $\left|\mathcal{V}_{\Delta}\right|$, é finito. Então a $K$-álgebra $\mathcal{A}$ gerada pelos elementos $\left\{\lambda_{\Delta}(g): g \in G\right\}$ coincide com $K \Delta$.

Demonstração. Denote por $B_{1}, \ldots, B_{m}$ os vértices da componente $\Delta$ e seja $(B, h)$ um elemento arbitrário desta componente.

Sejam $t_{2}, \ldots, t_{k}$ os únicos índices $t_{i}$ para os quais $B \backslash B_{t_{i}} \neq \emptyset$ e escolha um elemento $x_{i} \in B \backslash B_{t_{i}}$, para cada $2 \leq i \leq k$. Por conveniência, defina também $x_{0}=x_{1}=1$ e $x_{k+1}=h^{-1}$, e então $A_{0}=\left\{x_{1}, x_{2}, \ldots, x_{k+1}\right\} \subseteq B$.

Defina $g_{1}, g_{2}, \ldots, g_{k+1} \in G$ por meio da igualdade $g_{i}=x_{i}^{-1} x_{i-1}$, para $i \in \mathcal{I}=\{1, \ldots, k+1\}$. Em particular, tem-se $g_{1}=x_{1}=1$, bem como $g_{2}=x_{2}^{-1}$ e $g_{k+1}=h x_{k}$. Note que se $i \in \mathcal{I}$, então $g_{i}^{-1}$ 
pertence (pelo menos) ao suporte do vértice $\left(x_{i-1}^{-1} B, 1\right) \in \mathcal{V}_{\Delta}$. Consequentemente:

$$
\begin{aligned}
\lambda_{\Delta}\left(g_{k+1}\right) \cdots \lambda_{\Delta}\left(g_{1}\right)= & \sum_{\substack{g_{i}^{-1} \in A_{i} \in \mathcal{V}_{\Delta}, i \in \mathcal{I} \\
=}}\left(\sum_{\substack{g_{1}^{-1} \in A_{1} \in \mathcal{V}_{\Delta} \\
g_{2}^{-1} \in g_{1} A_{1}}}\left(A_{1}, g_{k+1} \cdots g_{1}\right)\right. \\
& \vdots \\
& \sum_{\substack{g_{k+1} \in g_{k} \ldots g_{1} A_{1} \\
g_{1}^{-1} \in A_{1} \in \mathcal{V}_{\Delta} \\
\left(g_{2} g_{1}\right)^{-1} \in A_{1}}}\left(A_{1}, h\right) . \\
& \vdots \\
& \left(g_{k+1} \cdots g_{1}\right)^{-1} \in A_{1}
\end{aligned}
$$

Como $g_{1}=1=x_{1}^{-1},\left(g_{2} g_{1}\right)=x_{2}^{-1}, \ldots,\left(g_{k+1} \cdots g_{1}\right)=h=x_{k+1}^{-1}$, tem-se que

$$
\lambda_{\Delta}\left(g_{k+1}\right) \cdots \lambda_{\Delta}\left(g_{1}\right)=\sum_{A_{0} \subseteq A \in \mathcal{V}_{\Delta}}(A, h)=\sum_{B \subseteq A \in \mathcal{V}_{\Delta}}(A, h) .
$$

A última igualdade em (2.8) deve-se ao fato de que $A_{0} \nsubseteq B_{t_{i}}$ para todo $i \in\{2, \ldots, k\}$, pela definição de $A_{0}$. Logo, todo $A$ nesta soma contém $B$.

A proposição será demonstrada por indução sobre $d(B)$, em que a função $d: \mathcal{V}_{\Delta} \rightarrow \mathbb{N} \cup\{0\}$ é definida a seguir. Se $A$ é maximal, isto é, se

$$
A \subseteq A^{\prime} \text {, para algum } A^{\prime} \in \mathcal{V}_{\Delta} \text {, implica } A=A^{\prime},
$$

será estipulado que $d(A)=0$. Caso contrário, será definido

$$
d(A)=\max \left\{n \in \mathbb{N} \mid \exists A_{1}, \ldots, A_{n} \in \mathcal{V}_{\Delta}, \operatorname{com} A_{n} \supsetneq \ldots \supsetneq A_{1} \supsetneq A\right\} .
$$

Se o suporte de $(B, h)$ se enquadra no primeiro caso, então $B$ não está contido no suporte de qualquer outro elemento de $\Delta$ e portanto a igualdade (2.8) reduz-se a

$$
\lambda_{\Delta}\left(g_{k+1}\right) \cdots \lambda_{\Delta}\left(g_{1}\right)=(B, h) .
$$

Portanto, a álgebra $\mathcal{A}$ contém todo $(B, h)$ cujo suporte é maximal.

Seja $d(B)=k>0$ e assuma por hipótese de indução que qualquer $(A, g) \in \Delta$ satisfazendo $0 \leq d(A)<k$ está em $\mathcal{A}$. Então escrevendo (2.8) na forma

$$
\lambda_{\Delta}\left(g_{k+1}\right) \cdots \lambda_{\Delta}\left(g_{1}\right)=(B, h)+\sum_{B \subsetneq A \in \mathcal{V}_{\Delta}}(A, h),
$$

nota-se que se $(A, h) \in \Delta$ e $B \subsetneq A$, então $d(A, h)<k=d(B, h)$. Por indução, tem-se $(A, h) \in \mathcal{A}$ e, consequentemente,

$$
(B, h)=\lambda_{\Delta}\left(g_{k+1}\right) \cdots \lambda_{\Delta}\left(g_{1}\right)-\sum_{B \subsetneq A \in \mathcal{V}_{\Delta}}(A, h) \in \mathcal{A} .
$$




\subsection{Interação entre Ações e Representações Parciais}

Seja $\pi$ uma representação parcial de $G$ em uma $K$-álgebra unital $\mathcal{B}$. Será utilizada a seguinte notação:

- $\varepsilon_{g}^{\pi}=\pi(g) \pi\left(g^{-1}\right)$, para cada $g \in G ;$

- $\mathcal{A}^{\pi}$ denotará a subálgebra de $\mathcal{B}$ gerada pelos $\varepsilon_{g}^{\pi}$;

- $\mathcal{D}_{g}^{\pi}=\varepsilon_{g}^{\pi} \mathcal{A}^{\pi}$, para cada $g \in G$.

Por simplicidade, caso $\pi$ esteja subentendida pelo contexto, as notações anteriores serão simplificadas para $\varepsilon_{g}, \mathcal{A}$ e $\mathcal{D}_{g}$, respectivamente.

Em [15, Lemma 6.5] é estabelecido o seguinte:

Lema 2.3.1. Dada uma representação parcial $\pi: G \rightarrow \mathcal{B}$, as aplicaçôes $\alpha_{g}^{\pi}: \mathcal{D}_{g^{-1}} \rightarrow \mathcal{D}_{g}$ dadas por $\alpha_{g}^{\pi}(a)=\pi(g) a \pi\left(g^{-1}\right)$ são isomorfismos de álgebras (para cada $g \in G$ ) e definem uma ação parcial $\alpha^{\pi}$ de $G$ em $\mathcal{A}$.

Reciprocamente, [15, Lemma 6.2] garante que:

Lema 2.3.2. Dada uma ação parcial $\alpha=\left\{\alpha_{g}: \mathcal{D}_{g^{-1}} \rightarrow \mathcal{D}_{g} \mid g \in G\right\}$ de $G$ em uma álgebra $\mathcal{A}$, tal que cada $\mathcal{D}_{g}$ é uma álgebra com unidade $1_{g}$, a aplicação $\pi_{\alpha}: G \rightarrow \mathcal{A} \rtimes_{\alpha} G$ definida por $\pi_{\alpha}(g)=1_{g} \delta_{g}$ é uma representação parcial.

Pode-se considerar a categoria $\operatorname{Rp} G$ cujos objetos são as representações parciais de um grupo $G$ (em álgebras com unidade) e cujos morfismos são dados pela Definição 1.2.3.

Analogamente, tem-se uma categoria $\operatorname{Ap} G$ em que os objetos são as ações parciais de um grupo $G$ em álgebras unitais ${ }^{1}$ e os morfismos são dados pela Definição 1.1.2, ou seja, dadas ações parciais $\alpha=\left\{\alpha_{g}: \mathcal{D}_{g^{-1}} \rightarrow \mathcal{D}_{g} \mid g \in G\right\}$ em $\mathcal{A}$ e $\alpha^{\prime}=\left\{\alpha_{g}^{\prime}: \mathcal{D}_{g^{-1}}^{\prime} \rightarrow \mathcal{D}_{g}^{\prime} \mid g \in G\right\}$ em $\mathcal{A}^{\prime}$, um morfismo de $\alpha$ em $\alpha^{\prime}$ é um homomorfismo de álgebras $\varphi: \mathcal{A} \rightarrow \mathcal{A}^{\prime}$ tal que, para todo $g \in G$ :

(i) $\varphi\left(\mathcal{D}_{g}\right) \subseteq \mathcal{D}_{g}^{\prime}$;

(ii) $\alpha_{g}^{\prime}(\varphi(x))=\varphi\left(\alpha_{g}(x)\right)$, para todo $x \in \mathcal{D}_{g^{-1}}$.

Pode-se definir uma subcategoria ApGl $G$ (não plena) de $\operatorname{Ap} G$ em que os objetos são somente as ações parciais globalizáveis do grupo $G$ (em álgebras com unidade). Se $\alpha, \alpha^{\prime}$ são duas de tais ações parciais, o conjunto $\operatorname{Hom}_{\mathbf{A p G l} G}\left(\alpha, \alpha^{\prime}\right)$ é formado pelos morfismos $\varphi \in \operatorname{Hom}_{\mathbf{A p} G}\left(\alpha, \alpha^{\prime}\right)$ que verificam a seguinte propriedade adicional:

(iii) $\varphi\left(1_{g}\right)=1_{g}^{\prime}, \forall g \in G$.

Aqui, $1_{g}$ e $1_{g}^{\prime}$ denotam as unidades dos ideais $\mathcal{D}_{g}$ e $\mathcal{D}_{g}^{\prime}$, respectivamente.

Seja $\alpha=\left\{\alpha_{g}: \mathcal{D}_{g^{-1}} \rightarrow \mathcal{D}_{g} \mid g \in G\right\}$ uma ação parcial globalizável de $G$ em $\mathcal{A}$, e $\mathcal{D}_{g}=\mathcal{A} 1_{g}$, para cada $g \in G$. Denote por $\tilde{\mathcal{A}}$ a subálgebra de $\mathcal{A}$ gerada pelas unidades $1_{g}$. Se $\mathcal{A}=\tilde{\mathcal{A}}$, a ação parcial $\alpha$ será denominada ajustada. Caso contrário, definindo-se $\tilde{\mathcal{D}}_{g}=\tilde{\mathcal{A}} 1_{g}$, para $g \in G$, tem-se $\tilde{\mathcal{D}}_{g} \subseteq \mathcal{D}_{g}$ e as restrições $\tilde{\alpha}_{g}=\left.\alpha_{g}\right|_{\tilde{\mathcal{D}}_{g^{-1}}}: \tilde{\mathcal{D}}_{g^{-1}} \rightarrow \tilde{\mathcal{D}}_{g}$ são isomorfismos que definem uma ação parcial (ajustada) $\tilde{\alpha}$ de $G$ em $\tilde{\mathcal{A}}$. Tal $\tilde{\alpha}$ será chamada de ajustamento de $\alpha$. A categoria das ações parciais (globalizáveis) ajustadas e seus morfismos será denotada por $\mathbf{A p A j} G$. Assim, $\mathbf{A p A j} G$ é uma subcategoria plena de ApGlG.

Uma representação $\pi: G \rightarrow \mathcal{B}$ é separadora se a soma $\sum_{g \in G} \mathcal{D}_{g}^{\pi} \pi(g)$ é direta, ou seja, se para todo $g, h \in G$ tem-se $\mathcal{D}_{g}^{\pi} \pi(g) \cap \sum_{h \neq g} \mathcal{D}_{h}^{\pi} \pi(h)=0$.

Como antes, $\mathcal{D}_{g}^{\pi}=\varepsilon_{g}^{\pi} \mathcal{A}^{\pi}, \forall g \in G$. A representação $\pi$ será denominada ajustada se $\mathcal{B}$ coincidir com a álgebra $\langle\pi(G)\rangle$, gerada pelos elementos $\pi(g)$, em que $g \in G$.

Considerando-se as representações parciais ajustadas e seus morfismos, obtém-se uma subcategoria plena de $\mathbf{R} \mathbf{p} G$ que será denotada por $\mathbf{R} \mathbf{p A j} G$. Restringindo-se às representações parciais

\footnotetext{
${ }^{1}$ Pode-se definir uma categoria como esta para álgebras sem unidade porém, neste texto, não será necessária essa generalidade.
} 
ajustadas separadoras e seus morfismos, tem-se uma subcategoria plena que será denotada por RpAjSe $G$.

O teorema a seguir descreve a interação entre essas categorias, de forma análoga à que foi feita em [20, Theorem 3].

\section{Teorema 2.3.3.}

(i) Existe um funtor $\alpha^{\diamond}: \boldsymbol{R} \boldsymbol{p} G \rightarrow \boldsymbol{A} \boldsymbol{p A j} G$ que leva cada $\pi \in O b \boldsymbol{R} \boldsymbol{p} G$ em $\alpha^{\pi}$ como no Lema 2.3.1.

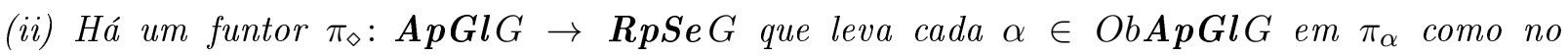
Lema 2.3.2. Além disso, $\pi_{\alpha}$ é ajustada se $\alpha$ é ajustada.

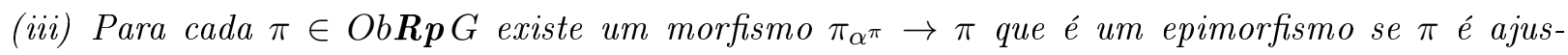
tada e é um monomorfismo se $\pi$ é separadora. Em particular, ele é um isomorfismo se $\pi \in O b R \boldsymbol{P A j S e} G$.

(iv) Para cada $\alpha \in$ ObApGlG existe um monomorfismo $\alpha^{\pi_{\alpha}} \rightarrow \alpha$, que é um isomorfismo se $\alpha$ é ajustada. Em qualquer caso tem-se $\alpha^{\pi_{\alpha}} \simeq \tilde{\alpha}$, em que $\tilde{\alpha}$ é o ajustamento de $\alpha$.

(v) As restriçôes dos funtores dos itens i e ii estabelecem uma equivalência entre as categorias RpAjSe G e ApAjG.

(vi) A restrição $\boldsymbol{R} \boldsymbol{p} \boldsymbol{A j} G \rightarrow \boldsymbol{A} \boldsymbol{p} \boldsymbol{A j} G$ do funtor obtido no item i é um adjunto à direita da restrição

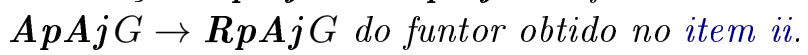

Demonstração. (i) Se $(\pi, \mathcal{B}) \in O b \mathbf{R p} G$, então pelo Lema 2.3.1 tem-se uma ação parcial $\alpha^{\diamond} \pi=$ $\alpha^{\pi}=\left\{\alpha_{g}^{\pi}: \mathcal{D}_{g^{-1}} \rightarrow \mathcal{D}_{g} \mid g \in G\right\}$ de $G$ na álgebra $\mathcal{A} \subseteq \mathcal{B}$ gerada pelas unidades $\varepsilon_{g}(g \in G)$, em que cada $\mathcal{D}_{g}=\varepsilon_{g} \mathcal{A}$. Em particular, $\alpha^{\pi}$ é ajustada.

Resta mostrar como o funtor $\alpha^{\diamond}$ age nos morfismos. Para isso, considere um objeto $\left(\pi^{\prime}, \mathcal{B}^{\prime}\right) \in$ $O b \mathbf{R} \mathbf{p} G$ e um morfismo $\phi \in H_{o m} m_{\mathbf{R p} G}\left(\pi, \pi^{\prime}\right)$. Como antes, tem-se uma ação parcial $\alpha^{\diamond} \pi^{\prime}=$ $\alpha^{\pi^{\prime}}=\left\{\alpha_{g}^{\pi^{\prime}}: \mathcal{D}_{g^{-1}}^{\prime} \rightarrow \mathcal{D}_{g}^{\prime} \mid g \in G\right\}$, de $G$ na álgebra $\mathcal{A}^{\prime} \subseteq \mathcal{B}^{\prime}$ gerada pelos elementos $\varepsilon_{g}^{\prime}$, com $g \in G$, em que cada $\mathcal{D}_{g}^{\prime}=\varepsilon_{g}^{\prime} \mathcal{A}^{\prime}$. Uma vez que $\phi$ é um morfismo de representações, $\phi: \mathcal{B} \rightarrow \mathcal{B}^{\prime}$ é um homomorfismo de álgebras e $\phi \circ \pi=\pi^{\prime}$. Logo, os geradores de $\mathcal{A}$ são levados nos geradores de $\mathcal{A}^{\prime}$, pois para cada $g \in G$ tem-se

$$
\phi\left(\varepsilon_{g}\right)=\phi\left(\pi(g) \pi\left(g^{-1}\right)\right)=\phi(\pi(g)) \phi\left(\pi\left(g^{-1}\right)\right)=\pi^{\prime}(g) \pi^{\prime}\left(g^{-1}\right)=\varepsilon_{g}^{\prime} .
$$

Consequentemente, $\phi(\mathcal{A})=\mathcal{A}^{\prime}$ e também há uma correspondência entre os ideais dessas álgebras:

$$
\phi\left(\mathcal{D}_{g}\right)=\phi\left(\varepsilon_{g} \mathcal{A}\right)=\varepsilon_{g}^{\prime} \phi(\mathcal{A})=\varepsilon_{g}^{\prime} \mathcal{A}^{\prime}=\mathcal{D}_{g}^{\prime} .
$$

Além disso, considerando-se que para quaisquer $a \in \mathcal{D}_{g^{-1}}$ e $g \in G$ vale

$$
\phi\left(\alpha_{g}^{\pi}(a)\right)=\phi\left(\pi(g) a \pi\left(g^{-1}\right)\right)=\pi^{\prime}(g) \phi(a) \pi^{\prime}\left(g^{-1}\right)=\alpha_{g}^{\pi^{\prime}}(\phi(a)),
$$

obtém-se o morfismo $\alpha^{\diamond} \phi$ por meio da restrição $\phi: \mathcal{A} \rightarrow \mathcal{A}^{\prime}$.

(ii) Dada uma ação parcial $\alpha=\left\{\alpha_{g}: \mathcal{D}_{g^{-1}} \rightarrow \mathcal{D}_{g} \mid g \in G\right\}$ de $G$ em uma álgebra $\mathcal{A}$, tal que cada $\mathcal{D}_{g}$ é uma álgebra com unidade $1_{g}$, o Lema 2.3.2 garante que a aplicação $\pi_{\alpha}: g \mapsto 1_{g} \delta_{g}$ é uma representação parcial de $G$ em $\mathcal{A} \rtimes_{\alpha} G=\oplus_{g \in G} \mathcal{D}_{g} \delta_{g}$. Além disso, para cada $g \in G$ tem-se

$$
\mathcal{D}_{g} \delta_{g}=\left(\mathcal{D}_{g} 1_{g}\right) \delta_{g}=\left(\mathcal{D}_{g} \delta_{1}\right)\left(1_{g} \delta_{g}\right)=\left(\mathcal{D}_{g} \delta_{1}\right) \pi_{\alpha}(g),
$$

donde

$$
\mathcal{A} \rtimes_{\alpha} G=\oplus_{g \in G}\left(\mathcal{D}_{g} \delta_{1}\right) \pi_{\alpha}(g) .
$$


Por outro lado, para que a representação $\pi_{\alpha}$ seja separadora, é preciso que $\sum_{g \in G} \mathcal{D}_{g}^{\pi_{\alpha}} \pi_{\alpha}(g)$ seja uma soma direta, mas levando em conta que, para cada $g \in G$,

$$
\varepsilon_{g}^{\pi_{\alpha}}=\pi_{\alpha}(g) \pi_{\alpha}\left(g^{-1}\right)=1_{g} \delta_{g} \cdot 1_{g^{-1}} \delta_{g^{-1}}=\alpha_{g}\left(\alpha_{g^{-1}}\left(1_{g}\right) 1_{g^{-1}}\right) \delta_{1}=1_{g} \delta_{1},
$$

segue-se que

$$
\begin{aligned}
\mathcal{D}_{g}^{\pi_{\alpha}} & =\left(\mathcal{A} \rtimes_{\alpha} G\right)^{\pi_{\alpha}} \varepsilon_{g}^{\pi_{\alpha}} \\
& =\left\{\sum k_{i} \varepsilon_{g_{i_{1}}}^{\pi_{\alpha}} \ldots \cdot \varepsilon_{g_{i_{t}}}^{\pi_{\alpha}}\right\} \cdot \varepsilon_{g}^{\pi_{\alpha}} \\
& \stackrel{(2.10)}{=}\left\{\sum k_{i}\left(1_{g_{i_{1}}} \delta_{1}\right) \cdot \ldots \cdot\left(1_{g_{i_{t}}} \delta_{1}\right)\right\} \cdot 1_{g} \delta_{1} \\
& =\left\{\sum k_{i}\left(1_{g_{i_{1}}} \ldots 1_{g_{i_{t}}}\right) 1_{g}\right\} \delta_{1} \\
& \subseteq \mathcal{D}_{g} \delta_{1} .
\end{aligned}
$$

Assim, $\mathcal{D}_{g}^{\pi_{\alpha}} \pi_{\alpha}(g) \subseteq\left(\mathcal{D}_{g} \delta_{1}\right) \pi_{\alpha}(g)$ para qualquer $g \in G$ e, pela igualdade (2.9), conclui-se que $\pi_{\alpha}$ é separadora.

Se $\alpha^{\prime}$ também for um objeto de ApGl $G$ e $\varphi: \alpha \rightarrow \alpha^{\prime}$ for um morfismo de ações parciais, então $\varphi\left(\mathcal{D}_{g}\right) \subseteq \mathcal{D}_{g}^{\prime}$ e pode-se considerar a aplicação $\phi: \mathcal{A} \rtimes_{\alpha} G \rightarrow \mathcal{A}^{\prime} \rtimes_{\alpha^{\prime}} G$ dada por $\phi\left(a \delta_{g}\right)=\varphi(a) \delta_{g}^{\prime}$.

Observa-se que

$$
\phi\left(a \delta_{g} \cdot b \delta_{h}\right)=\phi\left(\alpha_{g}\left(\alpha_{g^{-1}}(a) b\right) \delta_{g h}\right)=\varphi\left(\alpha_{g}\left(\alpha_{g^{-1}}(a) b\right)\right) \delta_{g h}^{\prime}
$$

Por outro lado,

$$
\begin{aligned}
\phi\left(a \delta_{g}\right) \cdot \phi\left(b \delta_{h}\right) & =\varphi(a) \delta_{g}^{\prime} \cdot \varphi(b) \delta_{h}^{\prime} \\
& =\alpha_{g}^{\prime}\left(\alpha_{g^{-1}}^{\prime}(\varphi(a)) \varphi(b)\right) \delta_{g h}^{\prime} \\
& =\alpha_{g}^{\prime}\left(\varphi\left(\alpha_{g^{-1}}(a)\right) \varphi(b)\right) \delta_{g h}^{\prime} \\
& =\alpha_{g}^{\prime}\left(\varphi\left(\alpha_{g^{-1}}(a) b\right)\right) \delta_{g h}^{\prime} \\
& =\varphi\left(\alpha_{g}\left(\alpha_{g^{-1}}(a) b\right)\right) \delta_{g h}^{\prime}
\end{aligned}
$$

Assim, $\phi$ preserva a multiplicação. Além disso, $\phi\left(1_{\mathcal{A}} \delta_{1}\right)=\varphi\left(1_{\mathcal{A}}\right) \delta_{g}^{\prime}=1_{\mathcal{A}}^{\prime} \delta_{g}^{\prime}$, segue-se que $\phi$ é um morfismo na categoria de álgebras unitais. Além disso,

$$
\phi\left(\pi_{\alpha}(g)\right)=\phi\left(1_{g} \delta_{g}\right)=\varphi\left(1_{g}\right) \delta_{g}^{\prime}=1_{g}^{\prime} \delta_{g}^{\prime}=\pi_{\alpha^{\prime}}(g),
$$

portanto $\phi \in \operatorname{Hom}_{\mathbf{R p S e} G}\left(\pi_{\alpha}, \pi_{\alpha^{\prime}}\right)$.

Se $\alpha$ for ajustada, então as unidades $1_{g}$ geram $\mathcal{A}$ e dado qualquer $a \delta_{g} \in \mathcal{A} \rtimes_{\alpha} G, a \in \mathcal{D}_{g}$, $g \in G$, tem-se $a=\beta 1_{g}$, em que $\beta$ é um polinômio nas variáveis $1_{h}(h \in G, h \neq g)$, e

$$
a \delta_{g}=\left(\beta 1_{g}\right) \delta_{g}=\left(\beta \delta_{1}\right)\left(1_{g} \delta_{g}\right) .
$$

Além disso, tem-se $\left(1_{g_{1}} \ldots \cdot 1_{g_{k}}\right) \delta_{1}=1_{g_{1}} \delta_{1} \cdot \ldots \cdot 1_{g_{k}} \delta_{1}$ para quaisquer desses monômios, e como $1_{h} \delta_{1}=1_{h} \delta_{h} \cdot 1_{h^{-1}} \delta_{h^{-1}}=\pi_{\alpha}(h) \pi_{\alpha}\left(h^{-1}\right)$ para todo $h \in G$, tem-se que $a \delta_{g}$ é um polinômio nas variáveis $\pi_{\alpha}(h)$, com $h \in G$. Portanto, $\pi_{\alpha}$ é ajustada.

(iii) Seja $\pi: G \rightarrow \mathcal{B}$ um objeto de $\mathbf{R} \mathbf{p} G$. Conforme [15, Proposition 6.8], existe um homomorfismo de $K$-álgebras $\psi: \mathcal{A}^{\pi} \rtimes_{\alpha^{\pi}} G \rightarrow \mathcal{B}$ dado por $\psi\left(\sum_{g \in G} a_{g} \delta_{g}\right)=\sum_{g \in G} a_{g} \pi(g)$, tal que $\psi \circ \pi_{\alpha^{\pi}}=\pi$. Logo $\psi$ define um morfismo $\pi_{\alpha^{\pi}} \rightarrow \pi$.

Se $\pi$ é ajustada, a álgebra $\mathcal{B}$ é gerada pelos elementos $\pi(g)=\varepsilon_{g} \pi(g)=\psi\left(\varepsilon_{g} \delta_{g}\right)$, em que $g \in G$. Neste caso, $\psi$ é sobrejetora. 
Se $\psi\left(\sum_{g \in G} a_{g} \delta_{g}\right)=\psi\left(\sum_{g \in G} b_{g} \delta_{g}\right)$, então $\sum_{g \in G} a_{g} \pi(g)=\sum_{g \in G} b_{g} \pi(g)$. Assim, se $\pi$ é separadora, a soma dos $\mathcal{D}_{g}^{\pi} \pi(g)(g \in G)$ é direta e consequentemente $a_{g} \pi(g)=b_{g} \pi(g)$, para todo $g \in G$. Neste caso, $a_{g}=a_{g} \varepsilon_{g}=a_{g} \pi(g) \pi\left(g^{-1}\right)=b_{g} \pi(g) \pi\left(g^{-1}\right)=b_{g} \varepsilon_{g}=b_{g}$. Portanto, $\psi$ é injetora.

(iv) Seja $\alpha$ uma ação parcial globalizável de $G$ em $\mathcal{A}$ dada por $\left\{\alpha_{g}: \mathcal{D}_{g^{-1}} \rightarrow \mathcal{D}_{g} \mid g \in G\right\}$, em que $\mathcal{D}_{g}=\mathcal{A} 1_{g}, \forall g \in G$ e denote por $\tilde{\alpha}$ o ajustamento de $\alpha$. Conforme a fórmula (2.10), para cada $g \in G$ tem-se $\varepsilon_{g}^{\pi_{\alpha}}=1_{g} \delta_{1}$ e pelo Lema 2.3.1, $\alpha^{\pi_{\alpha}}$ é uma ação parcial de $G$ na álgebra $\tilde{\mathcal{A}} \delta_{1} \subseteq \mathcal{A} \rtimes_{\alpha} G$ gerada pelos $\varepsilon_{g}^{\pi_{\alpha}}=1_{g} \delta_{1}$.

A aplicação $\varphi: \tilde{\mathcal{A}} \delta_{1} \rightarrow \tilde{\mathcal{A}}$ dada por $\varphi\left(a \delta_{1}\right)=a$ é um isomorfismo de álgebras, e suas restrições a $\left(\tilde{\mathcal{A}} \delta_{1}\right) \varepsilon_{g}^{\pi_{\alpha}}=\left(\tilde{\mathcal{A}} \delta_{1}\right) 1_{g} \delta_{1}=\left(\tilde{\mathcal{A}} 1_{g}\right) \delta_{1}=\tilde{\mathcal{D}}_{g} \delta_{1}$ são isomorfismos $\tilde{\mathcal{D}}_{g} \delta_{1} \rightarrow \tilde{\mathcal{D}}_{g}$, para cada $g \in G$.

Além disso, para cada $a \in \tilde{\mathcal{D}}_{g}^{-1}$, vale

$$
\begin{aligned}
\alpha_{g}^{\pi_{\alpha}}\left(a \delta_{1}\right) & =\pi_{\alpha}(g) a \delta_{1} \pi_{\alpha}\left(g^{-1}\right)=1_{g} \delta_{g} \cdot a \delta_{1} \cdot 1_{g^{-1}} \delta_{g^{-1}} \\
& =1_{g} \delta_{g} \cdot a 1_{g^{-1}} \delta_{g^{-1}}=\alpha_{g}(a) \delta_{1} .
\end{aligned}
$$

Então $\varphi\left(\alpha_{g}^{\pi_{\alpha}}\left(a \delta_{1}\right)\right)=\varphi\left(\alpha_{g}(a) \delta_{1}\right)=\alpha_{g}(a)=\alpha_{g}\left(\varphi\left(a \delta_{1}\right)\right)$ e $\varphi$ determina um isomorfismo entre as ações parciais $\alpha^{\pi_{\alpha}}$ e $\tilde{\alpha}$. Consequentemente, a composição $\tilde{\mathcal{A}} \delta_{1} \stackrel{\varphi}{\rightarrow} \tilde{\mathcal{A}} \stackrel{i}{\rightarrow} \mathcal{A}$, em que $i$ denota a inclusão, determina um monomorfismo de $\alpha^{\pi_{\alpha}} \rightarrow \alpha$. Em particular, se $\alpha$ for ajustada tem-se um isomorfismo $\alpha^{\pi_{\alpha}} \rightarrow \tilde{\alpha}=\alpha$.

(v) Primeiramente, observe que o conjunto de morfismos entre dois objetos de ApAj $G$ possui no máximo um elemento, pois qualquer que seja $\varphi \in \operatorname{Hom}_{\mathbf{A p A j} G}\left(\alpha, \alpha^{\prime}\right)$, deve ocorrer $\varphi\left(1_{g}\right)=1_{g}^{\prime}$ para todo $g \in G$, e como as álgebras nas quais $\alpha$ e $\alpha^{\prime}$ agem são geradas pelas unidades $1_{g} \mathrm{e}$ $1_{g}^{\prime}$, respectivamente, o valor de $\varphi$ fica completamente determinado em todos os elementos da álgebra. Analogamente, cada $\operatorname{Hom}_{\mathbf{R p A j} G}\left(\pi, \pi^{\prime}\right)$ é vazio ou consiste de um único elemento, e consequentemente o mesmo vale para cada $\operatorname{Hom}_{\mathbf{R p A j S e} G}\left(\pi, \pi^{\prime}\right)$. Deste modo, a restrição do funtor $\alpha^{\diamond}$ obtido no item i (e do funtor $\pi_{\diamond}$ do item ii) é um funtor pleno e fiel. De fato, a restrição é fiel pois não há flechas paralelas $\pi \rightrightarrows \pi^{\prime}$ entre objetos arbitrários de $\mathbf{R p A j S e ~} G$. Por outro lado, para que a restrição seja um funtor pleno, todo elemento de $\operatorname{Hom}_{\mathbf{A p A j} G}\left(\alpha^{\pi}, \alpha^{\pi^{\prime}}\right)$ deve ser a imagem por $\alpha^{\diamond}$ de algum morfismo $\pi \rightarrow \pi^{\prime}$ de $\operatorname{RpAjSe} G$. Mas se $\alpha^{\pi} \rightarrow \alpha^{\pi^{\prime}}$ é o único morfismo entre esses objetos, pode-se aplicar o funtor $\pi_{\diamond}$ do item ii para obter um morfismo $\pi_{\alpha^{\pi}} \rightarrow \pi_{\alpha^{\pi^{\prime}}}$, e usar o item iii para construir um morfismo $\pi \rightarrow \pi_{\alpha^{\pi}} \rightarrow \pi_{\alpha^{\pi^{\prime}}} \rightarrow \pi^{\prime}$ cuja imagem por $\alpha^{\diamond}$ será $\alpha^{\pi} \rightarrow \alpha^{\pi^{\prime}}$.

Levando em conta o isomorfismo $\pi_{\alpha^{\pi}} \rightarrow \pi$ (ou $\alpha^{\pi_{\alpha}} \rightarrow \alpha$ ) obtido no item iii (item iv, respectivamente), segue que as categorias $\operatorname{RpAjSe} G$ e $\operatorname{ApAj} G$ são equivalentes.

(vi) Para cada $\pi \in O b \mathbf{R} \mathbf{p A j} G$ e cada $\alpha \in O b \mathbf{A p A j} G$ é preciso associar uma bijeção

$$
\eta_{\alpha, \pi}: \operatorname{Hom}_{\mathbf{R p A} \mathbf{j} G}\left(\pi_{\alpha}, \pi\right) \rightarrow \operatorname{Hom}_{\mathbf{A p A j} G}\left(\alpha, \alpha^{\pi}\right)
$$

que seja natural tanto em $\alpha$ quanto em $\pi$. Fixada $\alpha \in O b \mathbf{A p A j} G$, a naturalidade de $\eta_{\alpha, \pi}$ em $\pi$ é obtida mostrando-se que para quaisquer $\pi_{1}, \pi_{2} \in O b \mathbf{R p A j} G$ e todo morfismo $\phi: \pi_{1} \rightarrow \pi_{2}$ tem-se um diagrama comutativo

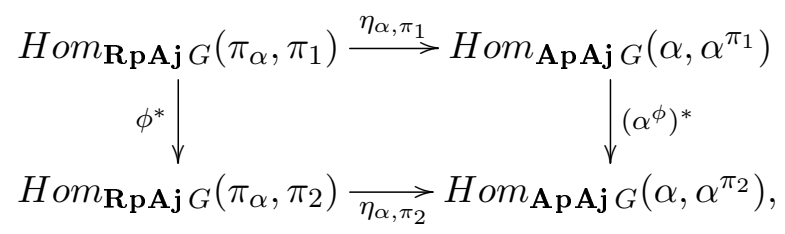

em que o morfismo $\alpha^{\phi}$ é a imagem de $\phi$ pelo funtor $\alpha^{\diamond}$, e as aplicações $\phi^{*}$ e $\left(\alpha^{\phi}\right)^{*}$ são induzidas pela composição com $\phi$ e $\alpha^{\phi}$, respectivamente. Por sua vez, a naturalidade em $\alpha$ corresponde 
à comutatividade de um diagrama análogo:

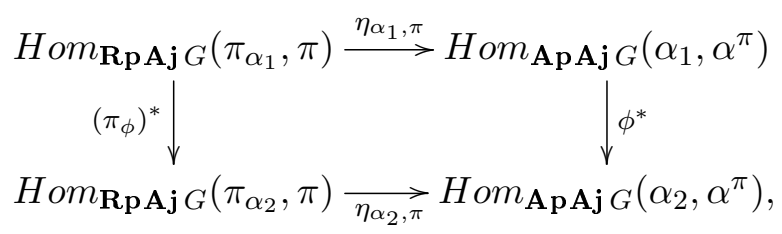

em que

- O morfismo $\phi: \alpha_{2} \rightarrow \alpha_{1}$ induz $\phi^{*}$ que leva $\psi \mapsto \psi \circ \phi$, para cada $\psi: \alpha_{1} \rightarrow \alpha^{\pi}$.

- O morfismo $\pi_{\phi}: \pi_{\alpha_{2}} \rightarrow \pi_{\alpha_{1}}$ induz $\left(\pi_{\phi}\right)^{*}$ que leva $\varphi \mapsto \varphi \circ \pi_{\phi}$, para cada $\varphi: \pi_{\alpha_{1}} \rightarrow \pi$.

Conforme a demonstração do item v, cada um dos conjuntos de morfismos do primeiro diagrama possui no máximo um único elemento. Então basta mostrar que $\operatorname{Hom}_{\mathbf{R p A j} G}\left(\pi_{\alpha}, \pi\right)=\emptyset$ exatamente quando $\operatorname{Hom}_{\mathbf{A p A} G G}\left(\alpha, \alpha^{\pi}\right)=\emptyset$, pois neste caso $\eta_{\alpha, \pi}$ ficará unicamente determinada e sua naturalidade em $\alpha$ e $\pi$ será imediata.

Suponha-se que $\operatorname{Hom}_{\mathbf{R p A j} G}\left(\pi_{\alpha}, \pi\right) \neq \emptyset$. Pelo item i o morfismo $\pi_{\alpha} \rightarrow \pi$ dá origem a um morfismo $\alpha^{\pi_{\alpha}} \rightarrow \alpha^{\pi}$. Por outro lado, o item iv garante a existência de um isomorfismo $\alpha^{\pi_{\alpha}} \rightarrow \alpha$, cujo inverso pode ser composto com $\alpha^{\pi_{\alpha}} \rightarrow \alpha^{\pi}$, resultando em um morfismo $\alpha \rightarrow \alpha^{\pi}$. Disso resulta que $\operatorname{Hom}_{\mathbf{A p A j} G}\left(\alpha, \alpha^{\pi}\right)$ também não é vazio.

Reciprocamente, se $\operatorname{Hom}_{\mathbf{A p A j} G}\left(\alpha, \alpha^{\pi}\right)$ não é vazio, o seu único elemento dá origem a um morfismo $\pi_{\alpha} \rightarrow \pi_{\alpha^{\pi}}$ (conforme o item ii). Além disso, o item iii garante a existência de um morfismo $\pi_{\alpha^{\pi}} \rightarrow \pi$, e a composição desses morfismos fornece um morfismo $\pi_{\alpha} \rightarrow \pi$. Disso resulta que $\operatorname{Hom}_{\mathbf{R p A j} G}\left(\pi_{\alpha}, \pi\right)$ também não é vazio.

Portanto, $\operatorname{Hom}_{\mathbf{R p A} \mathbf{j} G}\left(\pi_{\alpha}, \pi\right)=\emptyset \Leftrightarrow \operatorname{Hom}_{\mathbf{A p A j} G}\left(\alpha, \alpha^{\pi}\right)=\emptyset$ e a demonstração está completa. 


\section{Capítulo 3}

\section{Domínios de Representações Parciais Projetivas}

Neste capítulo demonstram-se alguns resultados sobre os domínios elementares, e é dada uma descrição dos domínios minimais e indecomponíveis. Também é mostrado que todo domínio pode ser decomposto de forma única como uma união de certos domínios indecomponíveis. Determina-se ainda a estrutura dos domínios elementares e obtêm-se alguns invariantes numéricos do conjunto parcialmente ordenado dos domínios elementares. Tais resultados permitem que seja obtida uma caracterização dos grupos para os quais todos os domínios elementares são indecomponíveis.

\subsection{Domínios Elementares}

Nesta seção introdutória, $G$ denotará um grupo finito. Seja $\operatorname{Pr}$ a projeção de $\oplus M_{m}(K H)$ sobre uma das álgebras de matrizes $M_{m}(K H)$, e considere a aplicação [ ]: $G \ni g \mapsto[g] \in K_{\mathrm{par}}(G)$.

Definição 3.1.1. Chama-se de representação parcial elementar de $G$ a uma função da forma

$$
\varphi=\operatorname{Pr} \circ \psi \circ[]: G \rightarrow M_{l}(K H),
$$

em que $\psi$ é o isomorfismo (2.1), que descreve a álgebra parcial de um grupo finito como uma soma de álgebras matriciais. ${ }^{1} \mathrm{O}$ conjunto

$$
D=D_{P r}=\{(x, y) \in G \times G \mid \varphi(x) \varphi(y) \neq 0\}
$$

é chamado de domínio elementar.

O algoritmo a seguir determina as representações parciais elementares de um grupo (finito) $G$. Para maiores detalhes, ver [16, seção 3] e [37, seção 2].

Dado um grupo $H$, a notação $e_{i, j}(h)$ será utilizada para indicar a matriz em $M_{m}(K H)$ cuja entrada na posição $(i, j)$ é $h$ e cujas demais entradas são 0. Além disso, também será utilizado $e_{i, j}=e_{i, j}\left(1_{H}\right)$.

Seja $A$ um vértice em $\Gamma(G)$, considere $H=\operatorname{St} A$ e escreva $A=\cup_{i=1}^{m} H g_{i}$ como uma união disjunta, em que $g_{1}=1$. Denote $\tau=\left\{g_{1}, \ldots, g_{m}\right\}$. O conjunto $\tau$ será chamado de transversal à direita de $H \mathrm{em} A$. A representação parcial elementar $\varphi_{A, \tau}: G \rightarrow M_{m}(K H)$ pode ser obtida da seguinte forma. Para qualquer $x \in G$ defina:

$$
\mathcal{I}_{x}^{A, \tau}=\left\{i \in\{1, \ldots, m\} \mid g_{i} x \in A\right\}
$$

Se $\mathcal{I}_{x}^{A, \tau}=\emptyset$ defina:

$$
\varphi_{A, \tau}(x)=0 .
$$

\footnotetext{
${ }^{1}$ Em [24] as representações parciais elementares foram definidas para grupos arbitrários.
} 
Se $\mathcal{I}_{x}^{A, \tau} \neq \emptyset$, então para cada $i \in \mathcal{I}_{x}^{A, \tau}$ existe um único elemento $j=j_{i, x}$ em $\{1, \ldots, m\}$ e $h=h_{i, x} \in$ $H$ tal que $g_{i} x=h g_{j}$. Neste caso temos

$$
\varphi_{A, \tau}(x)=\sum_{i \in \mathcal{I}_{x}^{A, \tau}} e_{i, j}(h)
$$

Denote por $D_{A, \tau}$ o domínio elementar que corresponde a $\varphi_{A, \tau}$.

Lema 3.1.2. Sejam $A$ e $A^{\prime}$ dois vértices da mesma componente conexa de $\Gamma(G)$. Considere também um transversal à direita $\tau$ de $\operatorname{St} A$ em $A$ e um transversal à direita $\tau^{\prime}$ de St $A^{\prime}$ em $A^{\prime}$. Então $D_{A, \tau}=D_{A^{\prime}, \tau^{\prime}}$.

Demonstração. Como $A^{\prime} \in \mathcal{V}_{A}$, existe uma aresta $\left(A^{\prime}, g\right) \in \Gamma(G)$ que vai de $\left(A^{\prime}, 1\right)$ para $(A, 1)=$ $\left(g A^{\prime}, 1\right)$, isto é, $A^{\prime}=g^{-1} A$. Uma vez que $g \in A=\cup_{i=1}^{m}$ St $A g_{i}$, existem $g_{k} \in \tau=\left\{g_{1}=1, g_{2}, \ldots, g_{m}\right\}$ e $h \in H=$ St $A$ tais que $g=h g_{k}$. Assim, tem-se $A^{\prime}=g_{k}^{-1} h^{-1} A=g_{k}^{-1} A$. Então $H^{\prime}=\operatorname{St} A^{\prime}=$ $g_{k}^{-1}(\mathrm{St} A) g_{k}$, e $\tau^{\prime \prime}=g_{k}^{-1} \tau$ é um transversal à direita de St $A^{\prime}$ em $A^{\prime}$. Assim, é fácil ver que a representação elementar $\varphi_{A^{\prime}, \tau^{\prime \prime}}: G \rightarrow M_{m}\left(K H^{\prime}\right)$ está relacionada a $\varphi_{A, \tau}: G \rightarrow M_{m}(K H)$ pela fórmula

$$
\varphi_{A^{\prime}, \tau^{\prime \prime}}(x)=\operatorname{diag}\left(g_{k}^{-1}, g_{k}^{-1}, \ldots g_{k}^{-1}\right) \varphi_{A, \tau}(x) \operatorname{diag}\left(g_{k}, g_{k}, \ldots g_{k}\right),
$$

para todo $x \in G$. Consequentemente, $D_{A, \tau}=D_{A^{\prime}, \tau^{\prime \prime}}$. Por [24, Lemma 3.1], as representações

$$
\varphi_{A^{\prime}, \tau^{\prime \prime}}, \varphi_{A^{\prime}, \tau^{\prime}}: G \rightarrow M_{m}\left(K H^{\prime}\right)
$$

são equivalentes, e disso decorre que $D_{A^{\prime}, \tau^{\prime \prime}}=D_{A^{\prime}, \tau^{\prime}}$. Conclui-se então que $D_{A, \tau}=D_{A^{\prime}, \tau^{\prime}}$.

Tendo em vista o Lema 3.1.2, $D_{A, \tau}$ e $\mathcal{I}^{A, \tau}$ serão denotados por $D_{A}$ e $\mathcal{I}^{A}$ respectivamente.

No próximo exemplo serão consideradas as duas possibilidades extremas para $A$.

Exemplo 3.1.3. Os casos em que $A=\{1\}$ ou $A=G$.

- Se $A=\{1\}$, então $H=\operatorname{St} A=\{1\}$ e tem-se a representação elementar $\varphi_{1}: G \rightarrow K$ dada por $\varphi_{1}(1)=1_{K}$ e $\varphi_{1}(x)=0$ para $x \neq 1$, e o domínio (elementar) correspondente é $\{(1,1)\}$.

- Se $A=G$, então $H=\operatorname{St} A=G$ e a representação elementar associada é $\varphi_{G}: G \ni x \rightarrow x \in K G$ com domínio (elementar) $G \times G$.

A lista a seguir mostra os domínios elementares dos grupos de ordem $\leq 4$, que serão utilizados posteriormente (veja também o Apêndice A). Denote por $C_{n}=\left\langle a \mid a^{n}=1\right\rangle$, o grupo cíclico de ordem $n \in \mathbb{N}$. Tem-se o seguinte:

Exemplo 3.1.4. Os domínios elementares dos grupos de ordem menor ou igual a 4 são os seguintes:

(i) Para $C_{1}$ o único domínio elementar é $\{(1,1)\}$.

(ii) Para $C_{2}$ os domínios elementares são:

- $C_{2} \times C_{2}$,

- $\{(1,1)\}$.

(iii) Para $C_{3}$ os domínios elementares são:

- $C_{3} \times C_{3}$,

- $D_{\{1, a\}}=\left\{(1,1),(1, a),\left(1, a^{-1}\right),(a, 1),\left(a^{-1}, 1\right),\left(a, a^{-1}\right),\left(a^{-1}, a\right)\right\}$,

- $\{(1,1)\}$.

(iv) Para $C_{4}$ os domínios elementares são:

- $C_{4} \times C_{4}$, 
- $D_{\{1, a\}}=\left\{(1,1),(1, a),\left(1, a^{-1}\right),(a, 1),\left(a^{-1}, 1\right),\left(a, a^{-1}\right),\left(a^{-1}, a\right)\right\}$,

- $D_{\left\{1, a^{2}\right\}}=\left\{(1,1),\left(1, a^{2}\right),\left(a^{2}, 1\right),\left(a^{2}, a^{2}\right)\right\}$,

- $\{(1,1)\}$.

(v) Para $C_{2} \times C_{2}=\left\langle a, b \mid a^{2}=b^{2}=(a b)^{2}=1\right\rangle$ os domínios elementares são:

- $\left(C_{2} \times C_{2}\right) \times\left(C_{2} \times C_{2}\right)$,

- $D_{\{1, a\}}=\{(1,1),(1, a),(a, 1),(a, a)\}$,

- $D_{\{1, b\}}=\{(1,1),(1, b),(b, 1),(b, b)\}$,

- $D_{\{1, a b\}}=\{(1,1),(1, a b),(a b, 1),(a b, a b)\}$,

- $\{(1,1)\}$.

De fato, os domínios elementares para os itens de (1) a (4) foram obtidos em [37, seção 3], enquanto que os domínios elementares de $C_{2} \times C_{2}$ podem ser obtidos facilmente por meio de (3.2) e (3.3).

Para encerrar esta seção, será estabelecido o seguinte:

Lema 3.1.5. Seja $H$ um subgrupo de $G$. Então todo domínio elementar de $H$ também é um domínio elementar de $G$.

Demonstração. Seja $D$ um domínio elementar de $H$ e $\varphi_{A, \tau}^{\prime}: H \rightarrow M_{l}\left(K H^{\prime}\right)$ uma representação parcial elementar de $H$ em que $H^{\prime}=\operatorname{St} A \subseteq H$ para algum vértice $A$ de $\Gamma(H), \tau$ é um transversal à direita de $H^{\prime}$ em $A$ e $D=\left\{(x, y) \in H \times H \mid \varphi_{A, \tau}^{\prime}(x) \varphi_{A, \tau}^{\prime}(y) \neq 0\right\}$. Então $A$ também é um vértice de $\Gamma(G)$ e definindo $\varphi: G \rightarrow M_{l}\left(K H^{\prime}\right)$ por

$$
\varphi(x)= \begin{cases}\varphi_{A, \tau}^{\prime}(x) & \text { se } x \in H \\ 0 & \text { se } x \notin H,\end{cases}
$$

é imediato que $D=\{(x, y) \in G \times G \mid \varphi(x) \varphi(y) \neq 0\}$ e conclui-se que $\varphi$ é a representação elementar $\varphi_{A, \tau} \operatorname{de} G$.

\subsection{Minimalidade e Indecomponibilidade}

Note que os subconjuntos $\mathcal{T}$-invariantes de $G \times G$ formam um reticulado completo $(C(G), \cap, \cup)$. Além disso, resulta do Exemplo 3.1.3 que $\{(1,1)\}$ e $G \times G$ são o zero e o neutro de $C(G)$, respectivamente. Os domínios $\{(1,1)\}$ e $G \times G$ serão chamados de domínios triviais de $G$. Observe também que todo domínio elementar de $G$ é um subconjunto $\mathcal{T}$-invariante de $G \times G$.

Para um grupo finito $G$, o Lema 3.1.2 implica que toda componente conexa de $\Gamma(G)$ corresponde a um domínio elementar, que pode ser obtido de um vértice arbitrário da componente. Será demonstrado que o domínio elementar associado ao vértice $A=\{1, a, b\}$ é a $\mathcal{T}$-órbita $\mathcal{T}\left(a^{-1}, b\right)$. Antes, no entanto, são necessários alguns resultados preliminares. Como no capítulo anterior, $\mathcal{V}_{A}$ denotará o conjunto dos vértices de $\Gamma(G)$ na componente que contém $A$.

Lema 3.2.1. Seja $G$ um grupo finito e $A$ um vértice de $\Gamma(G)$. Então $(x, y) \in D_{A}$ se, e somente se, existe $A^{\prime} \in \mathcal{V}_{A}$ tal que $\left\{x^{-1}, y\right\} \subseteq A^{\prime}$.

Demonstração. Seja $x, y \in G$. Por definição, $(x, y) \in D_{A}$ é equivalente a $\varphi_{A, \tau}(x) \varphi_{A, \tau}(y) \neq 0$, em que $\tau$ é um transversal à direita de St $A$ em $A$. Além disso, a igualdade $g_{i} x=h g_{j}$ vale se, e somente se, $g_{j} x^{-1}=h^{-1} g_{i}$, e consequentemente, utilizando (3.3), obtém-se

$$
\begin{aligned}
(x, y) \in D_{A} & \Leftrightarrow \exists j \in \mathcal{I}_{x^{-1}}^{A} \cap \mathcal{I}_{y}^{A} \\
& \Leftrightarrow g_{j} x^{-1} \in A \text { e } g_{j} y \in A \\
& \Leftrightarrow\left\{x^{-1}, y\right\} \subseteq g_{j}^{-1} A .
\end{aligned}
$$


O próximo resultado é uma consequência direta da definição de $\mathcal{T}$.

Lema 3.2.2. Se $G$ é um grupo arbitrário e $x \in G$, então

$$
\begin{aligned}
\mathcal{T}(x, 1) & =\left\{(1,1),(x, 1),\left(1, x^{-1}\right),\left(x^{-1}, x\right),\left(x^{-1}, 1\right),(1, x),\left(x, x^{-1}\right)\right\} \\
& =S_{3}(1,1) \cup S_{3}(x, 1) \cup S_{3}\left(x^{-1}, 1\right),
\end{aligned}
$$

$e$

$$
\mathcal{T}(x, 1)=\mathcal{T}\left(x^{-1}, 1\right)=\mathcal{T}\left(1, x^{-1}\right)=\mathcal{T}(1, x)=\mathcal{T}\left(x^{-1}, x\right)=\mathcal{T}\left(x, x^{-1}\right) .
$$

Agora será calculado o domínio elementar associado ao vértice $\{1, a, b\}$.

Proposição 3.2.3. Se $G$ é um grupo e $a, b \in G$ então:

$$
\mathcal{T}\left(b^{-1}, a\right)=\mathcal{T}(1, a) \cup \mathcal{T}(1, b) \cup \mathcal{T}\left(1, b^{-1} a\right) \cup S_{3}\left(b^{-1}, a\right) .
$$

Além disso, se $G$ é finito, então $D_{\{1, a, b\}}=\mathcal{T}\left(b^{-1}, a\right)$.

Demonstração. A igualdade (3.4) resulta do Lema 3.2.2 por meio de uma inspeção dos elementos de $\mathcal{T}\left(b^{-1}, a\right)$, com a ajuda da decomposição de $\mathcal{T}$ apresentada em (1.4) e da descrição das $S_{3}$-órbitas dada em (1.6). Por outro lado, observe que se $A=\{1, a, b\}$ então

$$
\mathcal{V}_{A}=\left\{A,\left\{1, a^{-1}, a^{-1} b\right\},\left\{1, b^{-1}, b^{-1} a\right\}\right\},
$$

havendo repetições se, e somente se, $A \cong C_{3}$ ou $|A| \leq 2$. Além disso, se $G$ é finito, a Proposição 3.2 .1 garante que um par $(u, v)$ pertence a $D_{\{1, a, b\}}$ se, e somente se,

$$
\left\{u^{-1}, v\right\} \subseteq\{1, a, b\} \text { ou }\left\{u^{-1}, v\right\} \subseteq\left\{1, a^{-1}, a^{-1} b\right\} \text { ou }\left\{u^{-1}, v\right\} \subseteq\left\{1, b^{-1}, b^{-1} a\right\} .
$$

Analisando caso a caso, obtém-se

$$
\begin{aligned}
D_{\{1, a, b\}}= & \left\{(1,1),(1, a),(1, b),\left(a^{-1}, 1\right),\left(a^{-1}, a\right),\left(a^{-1}, b\right),\left(b^{-1}, 1\right),\left(b^{-1}, a\right),\left(b^{-1}, b\right)\right\} \\
& \cup\left\{\left(1, a^{-1}\right),\left(1, a^{-1} b\right),(a, 1),\left(a, a^{-1}\right),\left(a, a^{-1} b\right),\left(b^{-1} a, 1\right),\left(b^{-1} a, a^{-1}\right),\left(b^{-1} a, a^{-1} b\right)\right\} \\
& \cup\left\{\left(1, b^{-1}\right),\left(1, b^{-1} a\right),(b, 1),\left(b, b^{-1}\right),\left(b, b^{-1} a\right),\left(a^{-1} b, 1\right),\left(a^{-1} b, b^{-1}\right),\left(a^{-1} b, b^{-1} a\right)\right\},
\end{aligned}
$$

e pelo Lema 3.2.2 e por (1.6), $D_{\{1, a, b\}}=\mathcal{T}(1, a) \cup \mathcal{T}(1, b) \cup \mathcal{T}\left(1, b^{-1} a\right) \cup S_{3}\left(b^{-1}, a\right)$.

Uma consequência imediata dessa proposição é o seguinte:

Corolário 3.2.4. As $\mathcal{T}$-órbitas de qualquer grupo finito $G$ são domínios elementares.

Definição 3.2.5. Se $G$ é um grupo arbitrário, um domínio $D \in C(G)$ será chamado decomponivel se existirem domínios $\left\{D_{i}\right\}_{i \in I}$, em que $D \neq D_{i} \neq D_{k} \neq D$ para $i \neq k$, tais que $D=\cup_{i \in I} D_{i}$. Caso contrário, $D$ será dito indecomponivel (ou $\cup$-irredutivel).

Observação 3.2.6. Em uma decomposição $D=\cup_{i \in I} D_{i}$ poderiam existir índices $j, k \in I$ tais que $D_{j} \subseteq D_{k}$. Neste caso, o domínio $D_{j}$ seria supérfluo. Assim, serão consideradas apenas decomposições minimais, no sentido de que $D_{j} \not \subseteq D_{k}$, para $j \neq k$.

Exemplo 3.2.7. Existe um domínio elementar decomponível para $C_{5}$. De fato foi calculado em [37, seção 3] que

$$
\begin{aligned}
& D_{4}=\left\{\left(1, C_{5}\right),\left(C_{5}, 1\right),(a, a),\left(a, a^{4}\right),\left(a^{4}, a\right),\left(a^{3}, a^{2}\right),\left(a^{2}, a^{3}\right),\left(a, a^{3}\right)\left(a^{3}, a\right),\right. \\
&\left.\left(a^{2}, a^{4}\right),\left(a^{4}, a^{2}\right),\left(a^{4}, a^{4}\right)\right\}=\mathcal{T}\left(a, a^{3}\right) \mathrm{e} \\
& D_{5}=\left\{\left(1, C_{5}\right),\left(C_{5}, 1\right),\left(a, a^{4}\right),\left(a^{4}, a\right),\left(a^{3}, a^{2}\right),\left(a^{2}, a^{3}\right),\left(a^{2}, a^{2}\right),\left(a, a^{2}\right),\left(a^{2}, a\right),\right. \\
&\left.\left(a^{3}, a^{4}\right),\left(a^{4}, a^{3}\right),\left(a^{3}, a^{3}\right)\right\}=\mathcal{T}\left(a^{2}, a^{2}\right)
\end{aligned}
$$

são domínios elementares, em que $\left(C_{5}, 1\right)$ e $\left(1, C_{5}\right)$ são a união dos pares $(x, 1)$ e $(1, x)$, para todo $x \in C_{5}$, respectivamente. Além disso, $C_{5} \times C_{5}=D_{4} \cup D_{5}$ e o domínio $C_{5} \times C_{5}$ é decomponível. 
Definição 3.2.8. Um domínio $D \neq\{(1,1)\}$ será dito minimal se, para todo domínio $D^{\prime}$, uma inclusão do tipo $D \supseteq D^{\prime} \supsetneq\{(1,1)\}$ implicar que $D=D^{\prime}$. Observe que os domínios minimais são precisamente os átomos do reticulado $C(G)$.

Note que na definição acima, se $G$ é finito pode-se assumir que $D^{\prime}$ é elementar, uma vez que [20, Theorem 4] garante que para grupos finitos todo domínio é a união de domínios elementares. Obviamente qualquer domínio minimal é indecomponível. Além disso, obtém-se o seguinte a partir do Lema 3.1.5:

Corolário 3.2.9. Se existe um dominio elementar decomponivel para um subgrupo $H$ de um grupo finito $G$, então também há um dominio elementar decomponivel para $G$.

Proposição 3.2.10. Se a e b são elementos quaisquer de $G$, então $\mathcal{T}(a, b)$ é um domínio indecomponivel (que é elementar se $G$ for finito), e todo domínio indecomponivel é uma $\mathcal{T}$-órbita.

Demonstração. Suponha que existam domínios (elementares se $G$ for finito) $D_{1}, \ldots, D_{k}$ tais que $\mathcal{T}(a, b)=\cup_{i=1}^{k} D_{i}$. Como $(a, b) \in \mathcal{T}(a, b)$, existe algum $j \in\{1, \ldots, k\}$ tal que $(a, b) \in D_{j}$. Mas $D_{j}$ também é invariante sob a ação de $\mathcal{T}$, então $\mathcal{T}(a, b) \subseteq D_{j} \subseteq \mathcal{T}(a, b)$, ou seja, $\mathcal{T}(a, b)$ coincide com o domínio elementar $D_{j}$. Portanto, $\mathcal{T}(a, b)$ é indecomponível.

Seja $G$ um grupo arbitrário. Então todo elemento $X \in C(G)$ é um subconjunto $\mathcal{T}$-invariante de $G \times G$, e é possível escrever $X=\cup_{(x, y) \in X} \mathcal{T}(x, y)$. Como todo $\mathcal{T}(x, y)$ contém $(1,1)$, é imediato que todo domínio minimal é uma $\mathcal{T}$-órbita. Mais especificamente, tem-se:

Proposição 3.2.11. Um dominio $D$ é minimal se, e somente se, $D=\mathcal{T}(x, 1)$, para algum $x \in$ $G \backslash\{1\}$.

Demonstração. $(\Leftarrow)$ Seja $x \in G \backslash\{1\}$ e suponha que $\{(1,1)\} \neq D^{\prime} \subseteq \mathcal{T}(x, 1)$, para algum domínio $D^{\prime}$. Então existe um par $(a, b) \in G \times G \operatorname{com}(1,1) \neq(a, b) \in D^{\prime} \subseteq \mathcal{T}(x, 1)$. Em particular, $(a, b) \in \mathcal{T}(x, 1)$ e utilizando o Lema 3.2.2, obtém-se $\mathcal{T}(x, 1) \subseteq D^{\prime} \subseteq \mathcal{T}(x, 1)$. Assim, $D^{\prime}=\mathcal{T}(x, 1) \mathrm{e}$ $\mathcal{T}(x, 1)$ é minimal.

$(\Rightarrow)$ Assuma que $D \neq \mathcal{T}(x, 1)$, para todo $x \in G \backslash\{1\}$. Se $D=\mathcal{T}(1,1)$, então obviamente $D$ não é minimal. Caso contrário, existe $(a, b) \in D$ tal que $a \neq 1$ ou $b \neq 1$. Além do mais,

- Se $a \neq 1$ então $\mathcal{T}(a, 1)=\mathcal{T} t(a, b) \subseteq D$;

- Se $b \neq 1$ então $\mathcal{T}\left(b^{-1}, 1\right)=\mathcal{T} \operatorname{th}(a, b) \subseteq D$.

Em ambos os casos, $D$ contém um domínio da forma $\mathcal{T}(x, 1) \neq\{(1,1)\}$, que por hipótese é diferente de $D$, contradizendo a minimalidade de $D$.

A combinação da Proposição 3.2.3 e da Proposição 3.2.11 resulta no seguinte:

Corolário 3.2.12. Seja $D$ um domínio para um grupo finito $G$. Então $D$ é minimal se, e somente se, existe $1 \neq x \in G$ tal que $D=D_{\{1, x\}}$.

Sabe-se que os domínios dos conjuntos fatores parciais são uniões de $\mathcal{T}$-órbitas. Uma $\mathcal{T}$-órbita não minimal será chamada de bloco. Consequentemente, para qualquer domínio $D$, existem blocos $\left\{B_{i} \mid i \in \mathcal{I}\right\}$ e átomos $\left\{M_{j} \mid j \in \mathcal{J}\right\}$ em $C(G)$ tais que

$$
D=\bigcup_{i \in \mathcal{I}} B_{i} \cup \bigcup_{j \in \mathcal{J}} M_{j}
$$

Também é possível remover de tal decomposição todo átomo $M_{j}$ que seja um subconjunto de algum $B_{i}$. Em outras palavras, pode-se assumir que neste tipo de decomposição de um domínio $D$ não existem $i, j$ de modo que $B_{i} \supseteq M_{j}$.

Proposição 3.2.13. Seja $G$ um grupo arbitrário. Suponha que os dominios $D_{1}, D_{2} \in C(G)$ estejam decompostos como

$$
D_{1}=\bigcup_{i \in \mathcal{I}} B_{i} \cup \bigcup_{j \in \mathcal{J}} M_{j}
$$


$e$

$$
D_{2}=\bigcup_{k \in \mathcal{K}} C_{k} \cup \bigcup_{l \in \mathcal{L}} N_{l}
$$

com $M_{j} \nsubseteq B_{i}$ e $N_{l} \nsubseteq C_{k}$, em que os $B_{i}^{\prime}$ s e os $C_{k}^{\prime}$ s são blocos e os $M_{j}^{\prime}$ s e os $N_{k}^{\prime}$ s são átomos. Se $D_{1} \subseteq D_{2}$, então:

- $\left\{B_{i} \mid i \in \mathcal{I}\right\} \subseteq\left\{C_{k} \mid k \in \mathcal{K}\right\}$;

- Para qualquer $j \in \mathcal{J}$ tem-se $M_{j} \subseteq C_{k}$, para algum $k \in \mathcal{K}$ ou $M_{j}=N_{l}$ para algum $l \in \mathcal{L}$.

Demonstração. Observe que a hipótese de que $M_{j} \nsubseteq B_{i}$ para cada $i$ implica $M_{j} \nsubseteq \bigcup_{i \in \mathcal{I}} B_{i}$, analogamente $N_{l} \nsubseteq \bigcup_{k \in \mathcal{K}} C_{k}$. Sabe-se pelo Lema 3.2.2 e pela Proposição 3.2.3 que, para qualquer $i$, $B_{i}=\mathcal{T}\left(x_{i}, y_{i}\right)$, para certos $x_{i}, y_{i} \in G \backslash\{1\}$ satisfazendo $1 \neq x_{i} y_{i}$. Por outro lado, $\left(x_{i}, y_{i}\right)$ é um elemento de

$$
\bigcup_{k \in \mathcal{K}} C_{k} \cup \bigcup_{l \in \mathcal{L}} N_{l}
$$

Além disso, $N_{j}=\mathcal{T}\left(1, a_{j}\right)$, para algum $a_{j}$ e cada elemento $(u, v)$ destas $\mathcal{T}$-órbitas satisfaz $1 \in$ $\{u, v, u v\}$. Consequentemente, $\left(x_{i}, y_{i}\right) \in C_{k}$, para algum $k$, e isso implica que $C_{k}=\mathcal{T}\left(x_{i}, y_{i}\right)=B_{i}$, e portanto $\left\{B_{i} \mid i \in \mathcal{I}\right\} \subseteq\left\{C_{k} \mid k \in \mathcal{K}\right\}$.

Em seguida, $M_{j}=\mathcal{T}\left(1, z_{j}\right)$, para algum $z_{j} \neq 1$, então se $M_{j}$ não está contido em nenhum dos blocos que aparecem na decomposição de $D_{2}$, nota-se que $M_{j} \subseteq \bigcup_{l \in \mathcal{L}} N_{l}$, para todo $j$. Como para cada $l$ tem-se $N_{l}=\mathcal{T}\left(1, z_{l}\right)$ para algum $z_{l} \neq 1$, resulta que $\left(1, z_{j}\right) \in \mathcal{T}\left(1, z_{l_{0}}\right)$ e $\mathcal{T}\left(1, z_{j}\right) \subseteq$ $\mathcal{T}\left(1, z_{l_{0}}\right)$, para algum $l_{0}$. Finalmente, pela Proposição 3.2.11, estas $\mathcal{T}$-órbitas são minimais, então $M_{j}=N_{l_{0}}$.

Corolário 3.2.14. Seja $G$ um grupo arbitrário. Se houver duas decomposições para um dominio $D \in C(G)$, como

$$
\bigcup_{i \in \mathcal{I}} B_{i} \cup \bigcup_{j \in \mathcal{J}} M_{j}
$$

$e$

$$
\bigcup_{k \in \mathcal{K}} C_{k} \cup \bigcup_{l \in \mathcal{L}} N_{l}
$$

com $M_{j} \nsubseteq B_{i}$ e $N_{l} \nsubseteq C_{k}$, em que os $B_{i}$ 's e os $C_{k}$ 's são blocos e os $M_{j}$ 's e os $N_{k}$ 's são átomos, então $\left\{B_{i} \mid i \in \mathcal{I}\right\}=\left\{C_{k} \mid k \in \mathcal{K}\right\}$ e $\left\{M_{j} \mid j \in \mathcal{J}\right\}=\left\{N_{l} \mid l \in \mathcal{L}\right\}$.

Demonstração. Pela proposição anterior, é claro que $\left\{B_{i} \mid i \in \mathcal{I}\right\}=\left\{C_{k} \mid k \in \mathcal{K}\right\}$. Disto, da hipótese de que $M_{j} \nsubseteq B_{i}$, e também da proposição anterior, decorre que $\left\{M_{j} \mid j \in \mathcal{J}\right\}=\left\{N_{l} \mid l \in \mathcal{L}\right\}$.

O próximo lema caracteriza os blocos contendo um átomo fixado em $C(G)$.

Lema 3.2.15. Seja $G$ um grupo arbitrário e a $\in G \backslash\{1\}$. Então $\mathcal{T}(1, a) \subseteq \mathcal{T}(x, y)$, com $1 \notin$ $\{x, y, x y\}$ se, e somente se, existe $z \in G$ tal que $\mathcal{T}(x, y)=\mathcal{T}(z, a)$.

Demonstração. Por (1.5) percebe-se que $(1, a) \in \mathcal{T}(z, a)$, para qualquer $z \in G$. Por outro lado, pela Proposição 3.2.3 tem-se $\mathcal{T}(x, y)=\mathcal{T}(1, y) \cup \mathcal{T}(1, x) \cup \mathcal{T}(1, x y) \cup S_{3}(x, y)$, e resulta de (1.6) que todo elemento $(a, b)$ de $S_{3}(x, y)$ é tal que $1 \notin\{a, b, a b\}$. Assim, se $\mathcal{T}(1, a) \subseteq \mathcal{T}(x, y)$, então $(1, a)$ pertence a $\mathcal{T}(1, x) \cup \mathcal{T}(1, y) \cup \mathcal{T}(1, x y)$, e segue do Lema 3.2 .2 que

$$
a \in\left\{x, x^{-1}, y, y^{-1}, x y, y^{-1} x^{-1}\right\} .
$$


Em outras palavras,

$$
\mathcal{T}(x, y)=\left\{\begin{array}{l}
\mathcal{T}(a, y)=\mathcal{T}\left(y^{-1} a^{-1}, a\right), \text { ou } \\
\mathcal{T}\left(a^{-1}, y\right)=\mathcal{T}\left(y^{-1}, a\right), \text { ou } \\
\mathcal{T}(x, a), \text { ou } \\
\mathcal{T}\left(x, a^{-1}\right)=\mathcal{T}\left(x a^{-1}, a\right), \text { ou } \\
\mathcal{T}\left(x, x^{-1} a\right)=\mathcal{T}\left(x^{-1}, a\right), \text { ou } \\
\mathcal{T}\left(x, x^{-1} a^{-1}\right)=\mathcal{T}\left(x^{-1} a^{-1}, a\right)
\end{array}\right.
$$

Em todo caso, tem-se $(1, a) \in \mathcal{T}(x, y)=\mathcal{T}(z, a)$, para algum $z \in G$.

Resulta da demonstração do Lema 3.2.15 o seguinte:

Corolário 3.2.16. Sejam $x, y \in G$. Para quaisquer $u, v \in G$ tem-se $\mathcal{T}(x, y) \cap \mathcal{T}(u, v)=\{(1,1)\}$ exatamente quando

$$
\{u, v, u v\} \cap\left\{x, x^{-1}, y, y^{-1}, x y, y^{-1} x^{-1}\right\}=\emptyset .
$$

\subsection{Decomposição de Domínios Elementares}

Nesta seção $G$ denotará um grupo finito. Pela Proposição 3.2.10 sabe-se que toda $\mathcal{T}$-órbita é um domínio elementar indecomponível, e também é conhecido que a união de $\mathcal{T}$-órbitas é um elemento de $C(G)$, apesar de não ser necessariamente um domínio elementar. Por exemplo, resulta do Exemplo 3.1.4 que para $C_{4}=\left\{1, a, a^{2}, a^{3}\right\}$ o domínio $X=\mathcal{T}(1, a) \cup \mathcal{T}\left(1, a^{2}\right)$ não é elementar. Como todo domínio elementar $D$ é uma união de $\mathcal{T}$-órbitas, $D=\cup_{(x, y) \in D} \mathcal{T}(x, y)$, é natural considerar o seguinte problema:

Dado um dominio elementar $D$, encontrar um conjunto $\mathcal{I}$ com uma quantidade minimal de elementos tal que $D=\bigcup_{i \in \mathcal{I}} \mathcal{T}\left(x_{i}, y_{i}\right)$.

Para resolver este problema será utilizado o seguinte:

Lema 3.3.1. Se $A$ e $B$ são vértices de $\Gamma(G)$ tais que $A \subseteq B$, então $D_{A} \subseteq D_{B}$.

Demonstração. Sejam $a, b \in G$. Pelo Lema 3.2.1 sabe-se que $(a, b) \in D_{A}$ é equivalente à existência de um elemento $g_{j} \in A \operatorname{com}\left\{a^{-1}, b\right\} \subseteq g_{j}^{-1} A$. Então existe algum $g_{j} \in B$ satisfazendo $\left\{a^{-1}, b\right\} \subseteq$ $g_{j}^{-1} B \in \mathcal{V}_{B}$. Novamente pelo Lema 3.2.1, conclui-se que $(a, b) \in D_{B}$.

Observação 3.3.2. A recíproca do Lema 3.3.1 não é verdadeira. Por exemplo, conforme [37, Corollary 6.2], se $|G|>3$ e $A$ é um vértice em $\Gamma(G)$, tal que $|A|+1=|G|$, então $D_{G}=D_{A}$.

Considere $G_{n}=\{A \subseteq G|1 \in A| A \mid,=n\}$ e defina uma relação de equivalência em $G_{n}$, por:

$$
A \sim B \Longleftrightarrow \mathcal{V}_{A}=\mathcal{V}_{B}
$$

A classe de equivalência de um vértice $A$ é exatamente $\mathcal{V}_{A}$.

Seja $\mathcal{C}_{n}$ um conjunto completo de representantes das classes de equivalência de $\sim$. O resultado a seguir fornece uma decomposição do domínio elementar induzido por um vértice $B \in \Gamma(G)$, em que $B$ tem 3 ou mais elementos. Pelos resultados já obtidos, para todo vértice $B$ satisfazendo $|B| \leq 3$, o domínio $D_{B}$ é uma $\mathcal{T}$-órbita.

Teorema 3.3.3. Seja $B$ um vértice em $\Gamma(G)$ tal que $|B| \geq 3$ e considere um transversal $\tau=\left\{b_{1}=\right.$ $\left.1, b_{2}, \ldots, b_{m}\right\} \subseteq B$ tal que $B=\bigcup_{i=1}^{m} \operatorname{St~} B b_{i}$. Então

$$
D_{B}=\bigcup_{i=1}^{m} \bigcup_{\substack{A \in \mathcal{C}_{3} \\ A \subseteq b_{i}^{-1} B}} D_{A} .
$$


Além disso, a decomposição acima é minimal, isto é, nenhum dos blocos que nela aparecem pode ser omitido, e a decomposição é única exceto por uma possivel permutação dos blocos.

Demonstração. Resulta do Corolário 3.3.1 e do Lema 3.1.2 que, para quaisquer $b \in B$ e $A \subseteq b^{-1} B$, tem-se $D_{A} \subseteq D_{b^{-1} B}=D_{B}$. Consequentemente, $D_{B} \supseteq \bigcup_{i=1}^{m} \bigcup_{\substack{A \in \mathcal{C}_{3} \\ A \subseteq b_{i}^{-1} B}} D_{A}$.

Para demonstrar a inclusão contrária, assuma que $(u, v) \in D_{B}$. Pelo Lema 3.2.1, há algum vértice $B^{\prime} \in \mathcal{V}_{B}$ que contém o conjunto $\left\{u^{-1}, v\right\}$. Como $\left|B^{\prime}\right|=|B| \geq 3$, existe um subconjunto $E$ de $B^{\prime}$ tal que $\left\{1, u^{-1}, v\right\} \subseteq E$ e $|E|=3$. Resulta novamente do Lema 3.2.1 que $(u, v) \in D_{E}$, e obtém-se (3.5).

A minimalidade da decomposição (3.5) será uma consequência das duas afirmações a seguir.

Afirmação 3.3.4. Se $A$ e $B_{1}$ são vértices em $\Gamma(G)$ tais que $|A|=\left|B_{1}\right|=3$, então

$$
\mathcal{V}_{A}=\mathcal{V}_{B_{1}} \Longleftrightarrow D_{A}=D_{B_{1}}
$$

De fato, já é conhecido do Lema 3.1.2 que todo vértice de uma determinada componente de $\Gamma(G)$ induz o mesmo domínio elementar. Para demonstrar a implicação contrária, sejam $A=\left\{1, a_{1}, a_{2}\right\}$ e $B_{1}=\left\{1, b_{1}, b_{2}\right\}$. Tem-se então:

$$
\begin{aligned}
\mathcal{V}_{A}=\mathcal{V}_{B_{1}} & \Longleftrightarrow B_{1}=A \text { ou } B_{1}=a_{1}^{-1} A \text { ou } B_{1}=a_{2}^{-1} A \\
& \Longleftrightarrow\left\{b_{1}, b_{2}\right\} \in\left\{\left\{a_{1}, a_{2}\right\},\left\{a_{1}^{-1}, a_{1}^{-1} a_{2}\right\},\left\{a_{2}^{-1}, a_{2}^{-1} a_{1}\right\}\right\} .
\end{aligned}
$$

Portanto, pela Proposição 3.2.3, é suficiente mostrar que $\mathcal{T}\left(a_{1}^{-1}, a_{2}\right)=\mathcal{T}\left(b_{1}^{-1}, b_{2}\right)$ implica a terceira condição em (3.6).

Assumindo $\mathcal{T}\left(a_{1}^{-1}, a_{2}\right)=\mathcal{T}\left(b_{1}^{-1}, b_{2}\right)$, tem-se $\left(b_{1}^{-1}, b_{2}\right) \in \mathcal{T}\left(a_{1}^{-1}, a_{2}\right)$, de modo que existe $\gamma \in \mathcal{T}=$ $S_{3} \cup t S_{3} \cup v t S_{3} \cup u v t S_{3} \cup 0$ tal que $\left(b_{1}^{-1}, b_{2}\right)=\gamma\left(a_{1}^{-1}, a_{2}\right)$. Além disso, por (1.5) e (1.6), resulta que

$$
t S_{3}(x, y)=\left\{(x, 1),(x y, 1),\left(y^{-1}, 1\right),\left(y^{-1} x^{-1}, 1\right),(y, 1),\left(x^{-1}, 1\right)\right\},
$$

para todo $(x, y) \in G \times G$. Assim, os pares $(a, b)$ de $\left(t S_{3} \cup v t S_{3} \cup 0\right)(x, y)$ possuem 1 em alguma de suas coordenadas. Como $1 \notin\left\{b_{1}, b_{2}\right\}$, resulta que $\gamma \notin t S_{3} \cup v t S_{3} \cup 0$. De modo similar, $\gamma \notin u v t S_{3}$, pois os pares em $u v t S_{3}\left(a_{1}^{-1}, a_{2}\right)$ são da forma $\left(x^{-1}, x\right)$, mas por hipótese $b_{1} \neq b_{2}$. Logo, a única possibilidade é que $\gamma \in S_{3}$. Neste caso, segue que $\left(b_{1}^{-1}, b_{2}\right)$ pertence ao conjunto

$$
\left\{\left(a_{1}^{-1}, a_{2}\right),\left(a_{1}^{-1} a_{2}, a_{2}^{-1}\right),\left(a_{2}^{-1}, a_{1}\right),\left(a_{2}^{-1} a_{1}, a_{1}^{-1}\right),\left(a_{2}, a_{2}^{-1} a_{1}\right),\left(a_{1}, a_{1}^{-1} a_{2}\right)\right\},
$$

o que é apenas uma forma alternativa de expressar a terceira condição de (3.6). Isso completa a demonstração desta afirmação.

Afirmação 3.3.5. Se $A, B_{1}, \ldots B_{m}$ são vértices pertencentes a componentes conexas distintas de $\Gamma(G), e|A|=\left|B_{j}\right|=3, j=1, \ldots, m$, então $D_{A} \nsubseteq \bigcup_{j=1}^{m} D_{B_{j}}$.

Fixe arbitrariamente um $j \in\{1, \ldots, m\}$ e escreva $A=\left\{1, a_{1}, a_{2}\right\}, B_{j}=\left\{1, b_{1}, b_{2}\right\}$. Então pela Afirmação 3.3.4 e pela Proposição 3.2.3 tem-se que $D_{A}=\mathcal{T}\left(a_{1}^{-1}, a_{2}\right) \neq \mathcal{T}\left(b_{1}^{-1}, b_{2}\right)=D_{B_{j}}$. Para $(x, y) \in \mathcal{T}\left(a_{1}^{-1}, a_{2}\right) \cap \mathcal{T}\left(b_{1}^{-1}, b_{2}\right)$ tem-se $\mathcal{T}(x, y) \subseteq \mathcal{T}\left(a_{1}^{-1}, a_{2}\right) \cap \mathcal{T}\left(b_{1}^{-1}, b_{2}\right)$. Por outro lado, pela Proposição 3.2.3 vê-se que os domínios não triviais contidos propriamente em uma $\mathcal{T}$-órbita são minimais. Como consequência, $1 \in\{x, y, x y\}$, e portanto, para todo $(u, v) \in D_{A}$ tal que $1 \notin$ $\{u, v, u v\}$ tem-se $(u, v) \notin D_{B_{j}}$. Em particular, $\left(a_{1}^{-1}, a_{2}\right) \notin D_{B_{j}}$. Como $j$ é arbitrário, resulta que $\left(a_{1}^{-1}, a_{2}\right) \notin \bigcup_{j=1}^{m} D_{B_{j}}$. Assim, $D_{A}=\mathcal{T}\left(a_{1}^{-1}, a_{2}\right) \nsubseteq \bigcup_{j=1}^{m} D_{B_{j}}$.

Finalmente, segue do Corolário 3.2.14 que a decomposição (3.5) é única.

Observação 3.3.6. Tem-se o seguinte: 
- O teorema anterior fornece um critério para decidir se um elemento $D \in C(G)$ é ou não um domínio elementar. De fato, seja $D=\bigcup_{i \in \mathcal{I}} \mathcal{T}\left(x_{i}, y_{i}\right)$ com $|\mathcal{I}| \geq 2$ e $B$ como no Teorema 3.3.3. Se existir $i \in \mathcal{I}$ tal que todo vértice em $\mathcal{V}_{\left\{1, y_{i}, x_{i}^{-1}\right\}}$ não esteja contido em $b_{j}^{-1} B$ para todo $1 \leq j \leq m$, tem-se $D \neq D_{B}$.

- Usando o Lema 3.2.2, pode-se reescrever a decomposição dada pela Proposição 3.2.3 da seguinte forma:

$$
D_{\{1, a, b\}}=S_{3}\left(b^{-1}, a\right) \cup \underset{x \in\left\{1, a, a^{-1}, b, b^{-1}, b^{-1} a, a^{-1} b\right\}}{ } S_{3}(x, 1),
$$

para quaisquer $a, b \in G$. Pelo Teorema 3.3.3, vê-se que todo domínio elementar é uma união de $S_{3}$-órbitas de $G \times G$, e consequentemente, todos os elementos de $C(G)$ são uniões de $S_{3}$-órbitas.

Definição 3.3.7. Diz-se que um vértice $A$ é total se $D_{A}=G \times G$.

A seguir serão apresentados alguns resultados com os quais é possível decidir quando o domínio induzido por um vértice de $\Gamma(G)$ não é total. Isto ajudará a caracterizar os grupos finitos para os quais todos os domínios elementares são indecomponíveis. Primeiro, observe a seguinte consequência direta do Teorema 3.3.3:

Corolário 3.3.8. Se $G$ é um grupo tal que $|G| \geq 3$ então

$$
D_{G}=G \times G=\bigcup_{A \in \mathcal{C}_{3}} D_{A} .
$$

Além disso, para todo vértice $B$ de $\Gamma(G)$, o dominio $D_{B}$ não é total, se, e somente se, existe $A \in \mathcal{C}_{3}$ tal que $D_{A}$ não está contido em $D_{B}$.

Proposição 3.3.9. Se $G$ é um grupo finito e $A$ é um vértice de $\Gamma(G)$ tal que $|\operatorname{St} A|\left(|G|^{2}-1\right)>$ $|A|\left(|A|^{2}-1\right)$, então $D_{A}$ não é total.

Demonstração. Pelo Lema 3.2.1, $(x, y) \in D_{A}$ exatamente quando existe algum vértice $A^{\prime} \in \mathcal{V}_{A}$ contendo $x^{-1}$ e $y$. Isso corresponde ao seguinte par de arestas no grupoide:

$$
y^{-1} A^{\prime} \stackrel{y}{\longrightarrow} A^{\prime} \stackrel{x}{\longrightarrow} x A^{\prime} .
$$

Para cada vértice $A^{\prime}$, existem exatamente $|A|^{2}$ pares distintos de tais arestas. Como um destes pares é sempre $(1,1)$, e $\left|\mathcal{V}_{A}\right|=|A| / \mid$ St $A \mid$, conclui-se que $D_{A}$ tem no máximo $\frac{|A|}{|\operatorname{St} A|}\left(|A|^{2}-1\right)+1$ elementos. Em particular, para qualquer $A$ que é total, tem-se

$$
|G|^{2}=\left|D_{A}\right| \leq \frac{|A|}{|\operatorname{St} A|}\left(|A|^{2}-1\right)+1
$$

Observação 3.3.10. As seguintes afirmações são verdadeiras para todo grupo finito $G$ :

- Para todo subgrupo $H \subsetneq G$, o domínio $D_{H}$ não é total.

- Se $A$ é um vértice de $\Gamma(G)$, em que $|G| \geq 6$ e $|A| \leq 3$, então $D_{A}$ não é total (basta usar a Proposição 3.3.9).

Proposição 3.3.11. Seja G um grupo finito. Então as seguintes condições são equivalentes,

(i) $1 \leq|G| \leq 4$,

(ii) todo domínio elementar de $G$ é indecomponivel, 
(iii) todos os dominios elementares não triviais de $G$ são minimais.

Demonstração. (i) $\Rightarrow$ (ii),(iii) Se $1 \leq|G| \leq 4$, resulta do Exemplo 3.1.4 que todo domínio elementar não trivial de $G$ induzido por $A \in \mathcal{V}_{A}$ é minimal e $D_{G}$ é indecomponível.

(ii) $\Rightarrow$ (i) Suponha que $|G|>4$. Se $|G| \geq 6$ então o segundo item da Observação 3.3 .10 e a Proposição 3.2.3 implicam que $D_{G}=G \times G$ não é uma $\mathcal{T}$-órbita, e, consequentemente, é decomponível. Finalmente, se $|G|=5$, o Exemplo 3.2.7 implica que neste caso existem domínios elementares decomponíveis para $G$.

(iii) $\Rightarrow$ (ii) Isso é evidente se $D_{G}$ é indecomponível. Por outro lado, se $D_{G}$ é decomponível, então pelo Exemplo 3.1.4, $|G| \geq 5$ e $G$ contém elementos $a \neq 1, b \neq 1$ com $a b \neq 1$. Neste caso o domínio $\mathcal{T}(a, b) \supsetneq \mathcal{T}(a, 1)$ não é minimal.

\subsection{Alguns Invariantes de $C(G)$}

Escreva $\mathcal{T}(G)=\{\mathcal{T}(x, y) \mid(x, y) \in G \times G\}$. Então $(\mathcal{T}(G), \subseteq)$ é um conjunto parcialmente ordenado cujos elementos maximais são os blocos, e pelo Corolário 3.2 .14 cada elemento de $C(G)$ pode ser escrito de forma única como uma união de elementos de $\mathcal{T}(G)$.

Proposição 3.4.1. Se $G$ e $H$ são grupos então $\mathcal{T}(G) \simeq \mathcal{T}(H)$ como conjuntos parcialmente ordenados se, e somente se, $C(G) \simeq C(H)$ como reticulados.

Demonstração. Seja $\phi: \mathcal{T}(G) \rightarrow \mathcal{T}(H)$ um isomorfismo de conjuntos parcialmente ordenados. Pelo Corolário 3.2.14 qualquer $D \in C(G)$ possui uma única decomposição $D=\cup_{i \in I} \mathcal{T}\left(a_{i}, b_{i}\right)$. Pode-se estender $\phi$ para $C(G)$ definindo

$$
\phi(D)=\cup_{i \in I} \phi\left(\mathcal{T}\left(a_{i}, b_{i}\right)\right) \in C(H) .
$$

Claramente, a decomposição de $\phi(D)$ acima é única. Assim, obtém-se uma correspondência um a um entre $C(G)$ e $C(H)$ que preserva inclusões, o que implica que tal correspondência é na verdade um isomorfismo de reticulados.

Reciprocamente, um isomorfismo de reticulados $\phi: C(G) \rightarrow C(H)$ leva domínios indecomponíveis de $G$ em domínios indecomponíveis de $H$, e como os domínios indecomponíveis são exatamente as $\mathcal{T}$-órbitas, ao restringir $\phi$ obtém-se um isomorfismo $\phi: \mathcal{T}(G) \rightarrow \mathcal{T}(H)$.

A seguir são calculados alguns invariantes relacionados aos domínios elementares minimais e aos blocos. O subconjunto dos elementos de $G$ cuja ordem é $n$ será denotado por $o_{n}(G)$, e lembre-se que um bloco é uma $\mathcal{T}$-órbita que não é minimal nem trivial. Note que a Proposição 3.2.3 implica que todo bloco contém um, dois ou três domínios elementares minimais. Será calculada a quantidade de blocos em cada um destes casos, bem como o número de domínios minimais.

Teorema 3.4.2. Seja G um grupo finito.

(i) O número $\min (G)$ de domínios elementares minimais de $G$ é dado por:

$$
\min (G)=\frac{|G|+\left|o_{2}(G)\right|-1}{2} .
$$

(ii) Denote por $\mathrm{Bl}_{1}(G)$ o conjunto de blocos que contêm exatamente um domínio elementar minimal. Então

$$
\left|\mathrm{Bl}_{1}(G)\right|=\mid\left\{H \text { subgrupo de } G \mid H \simeq C_{3}\right\}|=| o_{3}(G) \mid / 2 \text {. }
$$

(iii) Seja $\mathrm{Bl}_{2}(G)$ o conjunto dos blocos que contêm exatamente dois dominios elementares minimais. Então

$$
\left|\mathrm{Bl}_{2}(G)\right|=|\{a \in G \mid o(a)>3\}| / 2 .
$$


(iv) Escreva $\mathrm{Bl}(G)$ para indicar o conjunto de todos os blocos. Então

$$
|\mathrm{Bl}(G)|=\frac{\left(\begin{array}{c}
|G|-1 \\
2
\end{array}\right)+\left|o_{3}(G)\right|}{3} .
$$

(v) Seja $\mathrm{Bl}_{3}(G)$ o número de blocos que contêm exatamente três domínios elementares minimais. Então

$$
\left|\mathrm{Bl}_{3}(G)\right|=\frac{2\left(\begin{array}{c}
|G|-1 \\
2
\end{array}\right)-\left|o_{3}(G)\right|-3|\{a \in G \mid o(a)>3\}|}{6} .
$$

Demonstração. (i) Qualquer $x \in G \backslash\{1\}$, induz um domínio elementar $\mathcal{T}(x, 1)=\mathcal{T}\left(x^{-1}, 1\right)$, e a Proposição 3.2.11 garante que estes domínios são os únicos que são minimais. Por outro lado, pelo Lema 3.2.2 tem-se $\mathcal{T}(y, 1)=\mathcal{T}(x, 1)$ se, e somente se, $x=y$ ou $x=y^{-1}$. Consequentemente, o número de domínios elementares minimais $\mathcal{T}(x, 1)$ para os quais $x \in o_{2}(G)$ é exatamente $\left|o_{2}(G)\right|$. Por outro lado, cada subconjunto $\left\{y, y^{-1}\right\}$ de $G \backslash\left(o_{2}(G) \cup\{1\}\right)$ induz o domínio elementar minimal $\mathcal{T}(y, 1)$, e a quantidade de tais subconjuntos é $\frac{|G|-\left(\left|o_{2}(G)\right|+1\right)}{2}$. Portanto:

$$
\min (G)=\left|o_{2}(G)\right|+\frac{|G|-\left(\left|o_{2}(G)\right|+1\right)}{2}=\frac{|G|+\left|o_{2}(G)\right|-1}{2} .
$$

(ii) Para todo $a \in G$ tal que $o(a)=3$, obtém-se do Lema 3.2.2 e da Proposição 3.2.3 que $\mathcal{T}(a, a)=D_{\left\{1, a, a^{2}\right\}}=\mathcal{T}(1, a) \cup \mathcal{T}\left(1, a^{2}\right) \cup \mathcal{T}\left(1, a^{-2} a\right) \cup S_{3}\left(a^{-2}, a\right)=\mathcal{T}(1, a) \cup S_{3}(a, a)$. Assim, para cada $H=\left\langle a \mid a^{3}=1\right\rangle \subseteq G$ tem-se um bloco $\mathcal{T}(a, a)$ que contém exatamente um domínio elementar minimal. Além disso, do fato de que $a \neq b \neq a^{-1}$ implica $\mathcal{T}(1, a) \neq \mathcal{T}(1, b), a, b \in G$, segue que $\mathcal{T}(a, a) \neq \mathcal{T}(b, b)$ e essa correspondência é injetiva. Conclui-se então que $\left|\mathrm{Bl}_{1}(G)\right| \geq$ $\left|\left\{H<G \mid H \simeq C_{3}\right\}\right|$.

Por outro lado, se $D_{\{1, a, b\}}=\mathcal{T}(1, a) \cup \mathcal{T}(1, b) \cup \mathcal{T}\left(1, b^{-1} a\right) \cup S_{3}\left(b^{-1}, a\right)$ contém apenas um domínio elementar minimal, tem-se que $\mathcal{T}(1, a)=\mathcal{T}(1, b)=\mathcal{T}\left(1, b^{-1} a\right)$. Isso implica que $b=a^{-1}=b^{-1} a$, e disso conclui-se que $b=a^{2}$ e $a^{3}=1$. Portanto, $\{1, a, b\}=\left\{1, a, a^{2}\right\} \simeq C_{3}$ e conclui-se (ii).

(iii) Seja $\{1, a, b\}$ um conjunto de três elementos tais que

$$
D_{\{1, a, b\}}=\mathcal{T}(1, a) \cup \mathcal{T}(1, b) \cup \mathcal{T}\left(1, b^{-1} a\right) \cup S_{3}\left(b^{-1}, a\right)
$$

é um bloco contendo exatamente dois domínios elementares minimais. A componente de $\Gamma(G)$ gerada pelo vértice $\{1, a, b\}$ tem três vértices ou apenas um vértice. No último caso, pela prova do item (ii), $\{1, a, b\}$ é um grupo de ordem 3 cujo bloco associado está em $\mathrm{Bl}_{1}(G)$. Consequentemente, este é novamente o primeiro caso e $D_{\{1, a, b\}}=D_{\left\{1, a^{-1}, a^{-1} b\right\}}=D_{\left\{1, b^{-1}, b^{-1} a\right\}}$. Sem perda de generalidade, pode-se assumir que $\mathcal{T}(1, a)=\mathcal{T}(1, b) \neq \mathcal{T}\left(1, b^{-1} a\right)$. Isso implica que $b=a^{-1}$ e $b \neq b^{-1} a$. Então $a^{-1}=b \neq a^{2}$ e conclui-se que $o(a) \notin\{1,2,3\}$. Reciprocamente, é claro que todo bloco da forma $D_{\left\{1, a, a^{-1}\right\}}$, em que $a \in G$ é tal que $o(a)>3$ contém exatamente dois domínios minimais. Conclui-se que $\left|\mathrm{Bl}_{2}(G)\right|$ é igual à quantidade de subconjuntos $\left\{a, a^{-1}\right\}$ de $G$ para os quais $o(a)>3$. Esta última é $|\{a \in G \mid o(a)>3\}| / 2$, provando então (iii).

(iv) Como pelo Lema 3.1.2 vértices pertencentes a uma mesma componente conexa do grupoide $\Gamma(G)$ dão origem ao mesmo domínio elementar, só é preciso contar o número de componentes que são definidas por vértices $A$ de três elementos. Se um de tais vértices $A$ não é um grupo, então St $A=\{1\}$ e sua componente em $\Gamma(G)$ possui exatamente 3 vértices. Caso contrário, $A=$ St $A$ é um dos $\left|o_{3}(G)\right| / 2$ subgrupos de $G$ isomorfos a $C_{3}$. Assim, o número de blocos é

$$
\frac{\left|o_{3}(G)\right|}{2}+\frac{\left(\begin{array}{c}
|G|-1 \\
2
\end{array}\right)-\left|o_{3}(G)\right| / 2}{3}
$$

e resulta (iv).

(v) Pela Proposição 3.2.3, $|\mathrm{Bl}(G)|=\left|\mathrm{Bl}_{1}(G)\right|+\left|\mathrm{Bl}_{2}(G)\right|+\left|\mathrm{Bl}_{3}(G)\right|$, e assim (v) resulta de (ii), (iii) e (iv). 
Observação 3.4.3. Se $G$ é um grupo finito então, lembrando que as órbitas com 2 ou 6 elementos são chamadas de efetivas:

- Segue da prova do item (ii) do Teorema 3.4.2 que os blocos de $\operatorname{Bl}_{1}(G)$ correspondem àquelas componentes conexas de $\Gamma(G)$ que possuem exatamente 1 vértice $A$ tal que $|\operatorname{St}(A)|=3$, e também estão em correspondência biunívoca com as $S_{3}$-órbitas que contêm exatamente 2 elementos.

- Segue da Proposição 3.2.11 que as $S_{3}$-órbitas contendo exatamente 3 elementos correspondem aos domínios minimais de $G$, e os blocos correspondem às órbitas efetivas.

Exemplo 3.4.4. Seja $C_{n}=\left\langle a \mid a^{n}=1\right\rangle$ o grupo cíclico de ordem $n$. Se $3 \mid n$ então $a^{n / 3}$ e $a^{2 n / 3}$ são os únicos elementos de $C_{n}$ de ordem 3 , caso contrário $C_{n}$ não possui elementos de ordem 3 . Portanto, pelo item (iv) do Teorema 3.4.2,

$$
\left|\operatorname{Bl}\left(C_{n}\right)\right|=\frac{\left(\begin{array}{c}
n-1 \\
2
\end{array}\right)+\left|o_{3}(G)\right|}{3}= \begin{cases}\frac{(n-1)(n-2)}{6}, & \text { se } 3 \nmid n, \\
\frac{(n-1)(n-2)+4}{6}, & \text { se } 3 \mid n .\end{cases}
$$

Se $n \geq 4$ não é um múltiplo de 3 , então $\left|\operatorname{Bl}\left(C_{n}\right)\right|$ é igual ao número

$$
P_{n}=\left\lfloor\frac{n-1}{3}\right\rfloor\left(n-\frac{3}{2}\left\lfloor\frac{n-1}{3}\right\rfloor-\frac{3}{2}\right)
$$

obtido depois da prova de [21, Proposition 6.1]. De fato, é fácil verificar que $\left|\operatorname{Bl}\left(C_{n}\right)\right|=P_{n}$ se $n$ é da forma $3 k+1$ ou $3 k+2$, com $k \in \mathbb{N}$. No entanto, para $n=3 k$ o número de $S_{3}$-órbitas efetivas, que é o mesmo que o número de blocos, é na verdade $\left|\operatorname{Bl}\left(C_{n}\right)\right|=P_{n}+1$. Assim, [21, Proposition 6.1] deve ser reescrita da seguinte forma (que também leva em conta o caso $\left|\operatorname{Bl}\left(C_{3}\right)\right|=1$ ):

Proposição 3.4.5. Seja $n \geq 3$ e $\sigma \in$ pm $_{C_{n} \times C_{n}}^{\prime}\left(C_{n}\right)$. Então $\sigma$ é determinado unicamente por seus valores $\sigma(i, j)$, em que $1 \leq i \leq\left\lfloor\frac{n-1}{3}\right\rfloor, i \leq j \leq n-2 i-1$, e $\sigma\left(\frac{n}{3}, \frac{n}{3}\right)$ se $3 \mid n$.

Em vista do Exemplo 3.4.4, deve-se substituir $P_{n}$ por $\left|\operatorname{Bl}\left(C_{n}\right)\right|$ em [21, Corollary 6.2, Corollary 6.4]. Consequentemente, continua em aberto o problema de saber se para algum grupo $G$ existe alguma componente não total $p M_{D}(G)$ isomorfa à componente total $p M_{G \times G}(G)$.

Agora será considerada a questão de determinar o número de blocos $D_{\{1, x, y\}}$ diferentes que contém um certo domínio elementar minimal $\mathcal{T}(1, a)$. Um primeiro passo nesta direção foi mostrar no Lema 3.2.15 que os blocos do tipo $D_{\{1, a, z\}}$ esgotam a lista de blocos que contém a $\mathcal{T}$-órbita $\mathcal{T}(1, a)$. Ainda é preciso determinar se dois conjuntos distintos, por exemplo $\{1, a, y\}$ e $\{1, a, z\}$, podem dar origem ao mesmo bloco. O próximo resultado responde a este questionamento.

Lema 3.4.6. Se $|\{1, a, y, z\}|=4$ então $D_{\{1, a, y\}}=D_{\{1, a, z\}}$ se, e somente se, verifica-se uma das seguintes condiçôes:

- $a^{2}=1$ e $z=a y$; ou

- $\{y, z\}=\left\{a^{-1}, a^{2}\right\}$.

Demonstração. Conforme a Afirmação 3.3 .4 tem-se a igualdade $D_{\{1, a, y\}}=D_{\{1, a, z\}}$ se, e somente se, $\{1, a, z\}=\left\{1, a^{-1}, a^{-1} y\right\}$ ou $\{1, a, z\}=\left\{1, y^{-1}, y^{-1} a\right\}$. Isso ocorre exatamente quando $\{a, z\}=$ $\left\{a^{-1}, a^{-1} y\right\}$ ou $\{a, z\}=\left\{y^{-1}, y^{-1} a\right\}$, o que implica que $a^{2}=1$ e $z=$ ay ou $\{y, z\}=\left\{a^{-1}, a^{2}\right\}$.

Agora já é possível calcular o número de blocos nos quais está contido um certo domínio elementar minimal.

Proposição 3.4.7. Seja $a \in G \backslash\{1\}$ e denote por $\mathrm{Bl}(1, a)$ o conjunto dos blocos que contêm o dominio elementar minimal $\mathcal{T}(1, a)$. Então

$$
|\operatorname{Bl}(1, a)|= \begin{cases}(|G|-2) / 2, & \text { se } a^{2}=1, \\ |G|-2, & \text { se } a^{3}=1, \\ |G|-3, & \text { se } a^{2} \neq 1 \neq a^{3} .\end{cases}
$$


Demonstração. Será utilizado o Lema 3.4.6 levando em conta três casos.

- Se $a^{2}=1$, então para cada $y \in G \backslash\{1, a\}$, tem-se dois blocos idênticos, $D_{\{1, a, y\}}=D_{\{1, a, a y\}}$. Consequentemente, $|\mathrm{Bl}(1, a)|=(|G|-2) / 2$.

- Se $a^{3}=1$, a condição $\{y, z\}=\left\{a^{2}, a^{-1}\right\}$ só ocorre para $y=z$. Então $|\operatorname{Bl}(1, a)|=|G|-2$, neste caso.

- Se $a^{2} \neq 1 \neq a^{3}$, então $D_{\{1, a, y\}}=D_{\{1, a, z\}}$ para $y, z \in G \backslash\{1, a\}$ apenas se $\{y, z\}=\left\{a^{-1}, a^{2}\right\}$. Assim, $|\mathrm{Bl}(1, a)|=|G|-3$.

Exemplo 3.4.8. Considerando $G=C_{6}=\langle a\rangle$, e observando o diagrama dos domínios elementares correspondente na Figura A.6 do Apêndice A, note que:

- $o\left(a^{3}\right)=2$ e $\mathcal{T}\left(1, a^{3}\right)$ está contido em 2 blocos.

- $o\left(a^{2}\right)=3$ e $\mathcal{T}\left(1, a^{-2}\right)=\mathcal{T}\left(1, a^{2}\right)$ está contido em 4 blocos.

- $o(a)=6$ e $\mathcal{T}\left(1, a^{-1}\right)=\mathcal{T}(1, a)$ está contido em 3 blocos.

Tem-se o seguinte resultado:

Proposição 3.4.9. Se $\mathcal{T}(G) \simeq \mathcal{T}\left(G^{\prime}\right)$ (como conjuntos parcialmente ordenados) então $|G|=\left|G^{\prime}\right|$, $\left|o_{2}(G)\right|=\left|o_{2}\left(G^{\prime}\right)\right|$ e $\left|o_{3}(G)\right|=\left|o_{3}\left(G^{\prime}\right)\right|$.

Demonstração. Para todo grupo $G$ tem-se

$$
G=\{1\} \cup o_{2}(G) \cup o_{3}(G) \cup\{a \in G \mid o(a)>3\},
$$

e, pelo Teorema 3.4 .2 obtém-se

$$
|G|=1+\left|o_{2}(G)\right|+2\left|\mathrm{Bl}_{1}(G)\right|+2\left|\mathrm{Bl}_{2}(G)\right|,
$$

e as igualdades $\left|\mathrm{Bl}_{1}(G)\right|=\left|\mathrm{Bl}_{1}\left(G^{\prime}\right)\right|$ e $\left|\mathrm{Bl}_{2}(G)\right|=\left|\mathrm{Bl}_{2}\left(G^{\prime}\right)\right|$ implicam

$$
|G|-\left|o_{2}(G)\right|=\left|G^{\prime}\right|-\left|o_{2}\left(G^{\prime}\right)\right| \text {. }
$$

Além disso, o isomorfismo $\mathcal{T}(G) \simeq \mathcal{T}\left(G^{\prime}\right)$ também tem como consequência que $\min (G)=\min \left(G^{\prime}\right)$, e a proposição resulta do Teorema 3.4 .2

É natural considerar a seguinte questão: existem grupos não isomorfos $G$ e $H$ tais que $C(G) \simeq$ $C(H)$ ? Foram verificados alguns grupos de ordem pequena e até agora não foram encontrados grupos não isomorfos $G$ e $H \operatorname{com} C(G) \simeq C(H)$. Mas fica evidente pela Proposição 3.4 .9 que $C\left(C_{n}\right) \simeq C\left(C_{m}\right)$ implica $n=m$. 


\section{Capítulo 4}

\section{Multiplicador de Schur Parcial}

Em [21, Corollary 6.4] foi determinada a estrutura da componente total $p M_{C_{m} \times C_{m}}\left(C_{m}\right)$ do multiplicador de Schur parcial $p M\left(C_{m}\right)$ sobre um corpo algebricamente fechado $K$, em que $C_{m}$ é o grupo cíclico de ordem $m$. Neste capítulo serão apresentadas descrições análogas para algumas outras famílias de grupos $G$, como os grupos diedrais $D_{2 m}$ e os grupos dicíclicos $\mathrm{Dic}_{m}$. O mesmo problema também será considerado para os produtos diretos de grupos cíclicos finitos $C_{m} \times C_{n}$ e para alguns grupos infinitos (o cíclico infinito $\mathbb{Z}$ e o diedral infinito $D_{\infty}$ ).

De agora em diante será assumido que $K$ é algebricamente fechado. Em particular, tem-se um isomorfismo de grupos

$$
\frac{K^{*}}{\{1,-1\}} \simeq K^{*}
$$

Conforme foi lembrado na Observação 1.5.10, um conjunto fator parcial $\sigma \in \operatorname{pm}_{G \times G}^{\prime}(G)$ fica completamente determinado pelos seus valores em um conjunto completo de representantes das $S_{3}$-órbitas efetivas de $G$ (dadas pelas transformações (1.5)). Além disso, o número $s=s(G, G \times G)$ de $S_{3}$-órbitas efetivas de $G$ é igual ao número de $\mathcal{T}$-órbitas da forma $\mathcal{T}(a, b)$, em que $1 \notin\{a, b, a b\}$. De acordo com o Teorema 3.4.2,

$$
s(G, G \times G)=\frac{\left(\begin{array}{c}
|G|-1 \\
2
\end{array}\right)+\left|o_{3}(G)\right|}{3} .
$$

Em particular, se $G=D_{2 m}=C_{m} \rtimes C_{2}=\left\langle a, b \mid a^{m}=b^{2}=(a b)^{2}=1\right\rangle$ ou $G=C_{m} \times C_{2}=\langle a, b|$ $\left.a^{m}=b^{2}=[a, b]=1\right\rangle$, então $\left|o_{3}(G)\right|=2$, se 3||$G \mid$, e $\left|o_{3}(G)\right|=0$ caso contrário. Consequentemente,

$$
s(G, G \times G)= \begin{cases}\frac{(|G|-1)(|G|-2)+4}{6}, & \text { se } 3|| G \mid, \\ \frac{(|G|-1)(|G|-2)}{6}, & \text { se } 3 \nmid|G| .\end{cases}
$$

O Lema 4.0.11 fornecerá um conjunto completo de representantes das órbitas efetivas para esses grupos. Antes de prosseguir, observe que se $\sigma \in p m_{G \times G}^{\prime}(G)$, a igualdade (1.9) implica

$$
\sigma\left(a^{i} b, a^{j}\right)=\sigma\left(a^{k} b, a^{i} b\right)=\sigma\left(a^{j}, a^{k} b\right),
$$

em que o expoente $k=i-j$ se $G=D_{2 m}$ e $k=-i-j$ se $G=C_{m} \times C_{2}$.

Uma consequência imediata de (4.4) é que todo conjunto fator parcial $\sigma \in p m_{G \times G}^{\prime}(G)$ fica completamente determinado por seus valores naqueles pares cuja primeira coordenada é um elemento do subgrupo cíclico $C_{m}=\langle a\rangle$ de $G$. Assim, será conveniente adotar uma notação mais curta para esses valores, a saber

$$
\sigma_{i j}=\sigma\left(a^{i}, a^{j}\right) \text { e } \tau_{i j}=\sigma\left(a^{i}, a^{j} b\right) .
$$

Lema 4.0.10. Se $\sigma \in \mathrm{pm}_{C_{m} \times C_{m}}^{\prime}\left(C_{m}\right)$ e $m \geq 3$ então $\sigma$ fica completamente determinada por seus 
valores nos elementos de

$$
S_{C_{m}}=\left\{\left(a^{i}, a^{j}\right) \mid 1 \leq i \leq\lfloor(m-1) / 3\rfloor e i \leq j \leq m-2 i-1\right\} \cup Z_{a, m},
$$

em que $Z_{a, m}=\left\{\left(a^{\frac{m}{3}}, a^{\frac{m}{3}}\right)\right\}$ se $3 \mid m$ e $Z_{a, m}=\emptyset$ caso contrário. Além disso, esses valores podem ser escolhidos arbitrariamente em $K^{*}$.

Demonstração. Resulta da demonstração de [21, Proposition 6.1] que, para todo $m \geq 3$, o conjunto

$$
\left\{S_{3}\left(a^{i}, a^{j}\right) \mid 1 \leq i \leq\lfloor m / 3\rfloor, i \leq j \leq m-2 i\right\}
$$

contém todas as $S_{3}$-órbitas efetivas de $C_{m}$. Além disso, segue de $S_{3}\left(a^{i}, a^{i}\right)=S_{3}\left(a^{i}, a^{m-2 i}\right)$ que para cada inteiro $i$ satisfazendo $i<m-2 i$, existem dois representantes para a mesma $S_{3}$-órbita no conjunto acima. Nestes casos, $i<\frac{m}{3}$ e é suficiente considerar aquelas $S_{3}\left(a^{i}, a^{j}\right)$ tais que $j \leq m-2 i-1$ para obter um conjunto completo de representantes das $S_{3}$-órbitas efetivas.

A Figura 4.1 ilustra essa escolha de representantes para as órbitas efetivas no caso de grupos cíclicos.

Figura 4.1: Representantes das órbitas efetivas de $S_{3}$ em $C_{9} \times C_{9}$, em que $C_{9}=\left\langle a \mid a^{9}=1\right\rangle$.

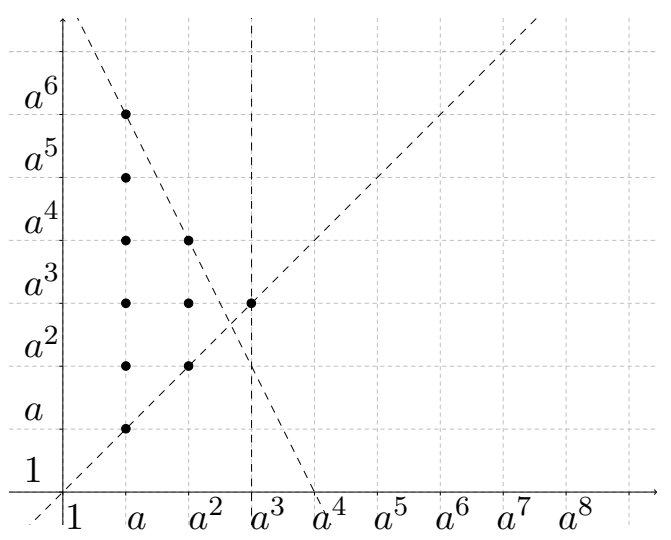

Lema 4.0.11. Seja $G=C_{m} \times C_{2}$ ou $G=D_{2 m}$, com $m \geq 3$, e $S_{C_{m}}$ como no Lema 4.0.10. Então qualquer $\sigma \in \mathrm{pm}_{G \times G}^{\prime}(G)$ fica completamente determinada por seus valores nos elementos de

$$
S_{G}=S_{C_{m}} \cup\left\{\left(a^{k}, a^{l} b\right) \mid 1 \leq k \leq\lfloor(m-1) / 2\rfloor e 0 \leq l \leq m-1\right\} \cup Z_{a, b, m},
$$

em que $Z_{a, b, m}=\left\{\left(a^{m / 2}, a^{l} b\right) \mid 0 \leq l \leq(m / 2)-1\right\}$ se $2 \mid m$ e $Z_{a, b, m}=\emptyset$ caso contrário. Além disso, esses valores podem ser escolhidos arbitrariamente em $K^{*}$.

Demonstração. Já é conhecido pelo Lema 4.0 .10 que a restrição de $\sigma$ a $C_{m} \times C_{m}$ fica completamente determinada por seus valores $\sigma_{i j}$, em que $\left(a^{i}, a^{j}\right) \in S_{C_{m}}$ e $S_{C_{m}}$ é dado por (4.6). Consequentemente, resta mostrar que cada um dos seus valores $\tau_{k l}$ é determinado por aqueles $\tau_{k l}$ cujos índices satisfazem as desigualdades em (4.7). Como $\sigma\left(1, a^{j} b\right)=1$, para todo $j$, é suficiente considerar $1 \leq k \leq m-1$ e $0 \leq l \leq m-1$.

Resulta de (1.6) (por inspeção) que $\left(a^{k}, a^{l} b\right)$ e $\left(a^{m-k}, a^{k+l} b\right)$ são os únicos possíveis pares da órbita $S_{3}\left(a^{k}, a^{l} b\right)$ cuja primeira coordenada é uma potência de $a$. Então, pode-se assumir sem perda de generalidade que o representante $\left(a^{k}, a^{l} b\right)$ foi escolhido de tal modo que $k$ é o menor expoente possível, em outras palavras, que $k \leq m-k$. Consequentemente, é suficiente considerar $1 \leq k \leq m / 2$.

Se existem $\left(a^{k}, a^{l} b\right) \neq\left(a^{k^{\prime}}, a^{l^{\prime}} b\right)$ na mesma $S_{3}$-órbita, com $1 \leq k \leq k^{\prime} \leq m / 2$ e $0 \leq l, l^{\prime} \leq m-1$ então, como foi visto acima, $\left(a^{k^{\prime}}, a^{l^{\prime}} b\right)=\left(a^{m-k}, a^{k+l} b\right)$. Portanto $a^{k^{\prime}}=a^{m-k}$ implica $k^{\prime}=k=m / 2$ (em particular, isso só ocorre se $m$ for par) e $a^{l^{\prime}} b=a^{m / 2+l} b$ implica $l^{\prime} \equiv m / 2+l(\bmod m)$. Neste caso, se $l \geq m / 2$ então $l^{\prime}=l-m / 2 \leq m / 2-1$. Portanto, quando $k=m / 2$ é inteiro é sempre possível escolher $0 \leq l \leq m / 2-1$. 
Nos próximos resultados, dado $\sigma \in p m_{G \times G}^{\prime}(G)$, será utilizada a seguinte notação.

- Fixado um elemento $x \in G$ defina

$$
\pi_{j}=\pi_{j}(x)=\sigma(x, x) \sigma\left(x, x^{2}\right) \ldots \sigma\left(x, x^{j-1}\right),
$$

para cada inteiro $j \geq 2$.

- De forma mais geral, dados elementos arbitrários $x, y \in G$ e um inteiro $j \geq 1$, seja

$$
\sigma_{j}(x, y)=\sigma(x, y) \sigma(x, x y) \sigma\left(x, x^{2} y\right) \ldots \sigma\left(x, x^{j-1} y\right) .
$$

Convencione também que $\sigma_{0}(x, y)=1$ e note que $\sigma_{j-1}(x, x)=\pi_{j}(x)$.

Lema 4.0.12. Seja $C_{m}=\left\langle a \mid a^{m}=1\right\rangle$. Se $\sigma \in p_{C_{m} \times C_{m}}^{\prime}\left(C_{m}\right)$ e $\sigma \sim 1$, isto é, se existe $\rho: G \rightarrow K^{*}$ tal que $\sigma(x, y)=\rho(x) \rho(y) / \rho(x y)$, para quaisquer $x, y \in G$, então $\pi_{j}(a)=\rho^{j}(a) / \rho\left(a^{j}\right) e$

$$
\sigma\left(a^{i}, a^{j}\right)=\frac{\pi_{i+j}}{\pi_{i} \pi_{j}}=\frac{\sigma\left(a, a^{i}\right) \ldots \sigma\left(a, a^{i+j-1}\right)}{\sigma(a, a) \ldots \sigma\left(a, a^{j-1}\right)}
$$

para todo $\left(a^{i}, a^{j}\right) \in S_{C_{m}}$ tal que $i, j \geq 2$. Além disso,

$$
\sigma\left(a, a^{j}\right)=\sigma\left(a, a^{m-j-1}\right),
$$

para qualquer $\left(a, a^{j}\right) \in S_{C_{m}}$ que satisfaça $\lfloor(m+1) / 2\rfloor \leq j \leq m-3$.

Demonstração. A justificativa é bastante simples e aparece na prova de [21, Proposition 6.3].

A igualdade (4.13) do lema a seguir é uma versão um pouco mais geral de (4.10).

Lema 4.0.13. Dado $\sigma \in p m_{G \times G}^{\prime}(G)$, se existe $\rho: G \rightarrow K^{*}$ tal que $\sigma(x, y)=\rho(x) \rho(y) / \rho(x y)$, para quaisquer $x, y \in G$, então

$$
\begin{gathered}
\sigma_{j}(x, y)=\frac{\rho^{j}(x) \rho(y)}{\rho\left(x^{j} y\right)}, \\
\sigma\left(x^{i}, x^{k} y\right)=\frac{\sigma_{i+k}(x, y) \sigma\left(x^{i}, y\right)}{\sigma_{i}(x, y) \sigma_{k}(x, y)},
\end{gathered}
$$

para quaisquer $i, j, k \geq 1$ e $x, y \in G$. Além disso, para quaisquer $x, y \in G$ e $i, k \in \mathbb{Z}$,

$$
\sigma\left(x^{i}, x^{k}\right)=\frac{\sigma\left(x^{i}, y\right) \sigma\left(x^{k}, x^{i} y\right)}{\sigma\left(x^{i+k}, y\right)} .
$$

Demonstração. As igualdades anteriores seguem diretamente das definições (como na prova de [21, Proposition 6.3]). Dados $i, j, k, x$ e $y$ como acima,

$$
\sigma_{j}(x, y)=\frac{\rho(x) \rho(y)}{\rho(x y)} \frac{\rho(x) \rho(x y)}{\rho\left(x^{2} y\right)} \frac{\rho(x) \rho\left(x^{2} y\right)}{\rho\left(x^{3} y\right)} \ldots \frac{\rho(x) \rho\left(x^{j-1} y\right)}{\rho\left(x^{j} y\right)}=\frac{\rho^{j}(x) \rho(y)}{\rho\left(x^{j} y\right)} .
$$

Consequentemente,

$$
\sigma\left(x^{i}, x^{k} y\right)=\frac{\rho\left(x^{i}\right) \rho\left(x^{k} y\right)}{\rho\left(x^{i+k} y\right)}=\frac{\rho^{i+k}(x) \rho(y)}{\rho\left(x^{i+k} y\right)} \frac{\rho\left(x^{i}\right) \rho(y)}{\rho\left(x^{i} y\right)} \frac{\rho\left(x^{i} y\right)}{\rho^{i}(x) \rho(y)} \frac{\rho\left(x^{k} y\right)}{\rho^{k}(x) \rho(y)}=\frac{\sigma_{i+k}(x, y) \sigma\left(x^{i}, y\right)}{\sigma_{i}(x, y) \sigma_{k}(x, y)} .
$$

Além disso,

$$
\sigma\left(x^{i}, x^{k}\right)=\frac{\rho\left(x^{i}\right) \rho\left(x^{k}\right)}{\rho\left(x^{i+k}\right)}=\frac{\rho\left(x^{i}\right) \rho(y)}{\rho\left(x^{i} y\right)} \frac{\rho\left(x^{k}\right) \rho\left(x^{i} y\right)}{\rho\left(x^{i+k} y\right)} \frac{\rho\left(x^{i+k} y\right)}{\rho\left(x^{i+k}\right) \rho(y)}=\frac{\sigma\left(x^{i}, y\right) \sigma\left(x^{k}, x^{i} y\right)}{\sigma\left(x^{i+k}, y\right)} .
$$




\subsection{Grupos Diedrais}

Nesta seção será determinada a componente total $p M_{G \times G}(G)$ do multiplicador de Schur parcial $p M(G)$ sobre um corpo algebricamente fechado $K$, para qualquer grupo diedral finito $G=D_{2 m}=$ $\left\langle a, b \mid a^{m}=b^{2}=(a b)^{2}=1\right\rangle$. Em particular, o Teorema 4.1.1 generalizará [38, Lemma 3.10], em que foi considerado o caso $m=3$.

Por simplicidade, seja $d_{m}=s\left(D_{2 m}, D_{2 m} \times D_{2 m}\right)$ o número de órbitas efetivas do grupo diedral $D_{2 m}$, que é dado pela fórmula (4.3).

Teorema 4.1.1. $p M_{D_{2 m} \times D_{2 m}}\left(D_{2 m}\right) \simeq\left(K^{*}\right)^{d_{m}-\left\lfloor\frac{m-1}{2}\right\rfloor}$.

Demonstração. Sejam $\psi:\left(K^{*}\right)^{d_{m}} \rightarrow p M_{D_{2 m} \times D_{2 m}}\left(D_{2 m}\right)$ o epimorfismo de grupos dado por (1.16) e $x \in \operatorname{ker}(\psi)=L_{D_{2 m} \times D_{2 m}}$. Então $\sigma=\sigma_{x} \in N_{D_{2 m} \times D_{2 m}}$. Isso significa que existe uma aplicação $\rho: D_{2 m} \rightarrow K^{*}$ tal que

$$
\sigma\left(a^{i}, a^{j}\right)=\frac{\rho\left(a^{i}\right) \rho\left(a^{j}\right)}{\rho\left(a^{i+j}\right)} \text { e } \sigma\left(a^{k}, a^{l} b\right)=\frac{\rho\left(a^{k}\right) \rho\left(a^{l} b\right)}{\rho\left(a^{k+l} b\right)},
$$

para quaisquer $i, j, k, l$, e $\rho$ também satisfaz

$$
\rho(1)=\rho\left(a^{i}\right) \rho\left(a^{-i}\right)=\rho\left(a^{i} b\right)^{2}=1,
$$

para todo $i$.

É fácil verificar que $\sigma^{2}\left(a^{k}, a^{l} b\right)=\rho^{2}\left(a^{k}\right)$ para quaisquer $k$ e $l$. Em particular, se $m$ é par então $\sigma^{2}\left(a^{\frac{m}{2}}, a^{l} b\right)=1$, para todo $l$. Resulta de (4.14) que, para qualquer $j=1, \ldots,\lfloor(m-1) / 2\rfloor$,

$$
\sigma\left(a, a^{j}\right)=\frac{\sigma(a, b) \sigma\left(a^{j}, a b\right)}{\sigma\left(a^{j+1}, b\right)}
$$

Portanto, usando os lemas 4.0.11 e 4.0.12, e indexando as coordenadas dos elementos de $\left(K^{*}\right)^{d_{m}}$ pelos representantes das órbitas efetivas correpondentes aos valores $\sigma_{i j}$ e $\tau_{k l}$ (definidos em (4.5)), fica claro que

$$
\begin{gathered}
\operatorname{ker}(\psi) \subseteq R=\left\{\left(\sigma_{i j}, \tau_{k l}\right) \in\left(K^{*}\right)^{d_{m}} \mid \sigma_{i j}=\frac{\sigma_{1 i} \ldots \sigma_{1, i+j-1}}{\sigma_{11} \ldots \sigma_{1, j-1}}, \text { para }(i, j) \in J_{1} ;\right. \\
\sigma_{1 j}=\sigma_{1, m-j-1}, \text { para } j \in J_{2} ; \sigma_{1 j}=\frac{\tau_{10} \tau_{j 1}}{\tau_{1+j, 0}}, \text { para } j \in J_{3} ; \\
\left.\tau_{k l}^{2}=\tau_{k 0}^{2}, \text { para }(k, l) \in J_{4} ; \tau_{\frac{m}{2}, l}^{2}=1, \text { para } l \in J_{5}\right\}
\end{gathered}
$$

onde

$$
\begin{aligned}
& J_{1}=\left\{(i, j) \in \mathbb{N} \times \mathbb{N} \mid i, j \geq 2 \text { e }\left(a^{i}, a^{j}\right) \in S_{C_{m}}\right\}, \\
& J_{2}=\{j \in \mathbb{N} \mid\lfloor(m+1) / 2\rfloor \leq j \leq m-3\}, \\
& J_{3}=\{j \in \mathbb{N} \mid 1 \leq j \leq\lfloor(m-1) / 2\rfloor\}, \\
& J_{4}=\{(i, j) \in \mathbb{N} \times \mathbb{N} \mid 1 \leq i \leq\lfloor(m-1) / 2\rfloor ; 0 \leq j \leq m-1\}, \\
& J_{5}= \begin{cases}\{l \in \mathbb{N} \mid 0 \leq l \leq(m / 2)-1\}, & \text { se } 2 \mid m, \\
\emptyset, & \text { se } 2 \nmid m .\end{cases}
\end{aligned}
$$


Note que $\sum_{i=1}^{5}\left|J_{i}\right|=d_{m}$. Agora, considere o grupo

$$
\begin{aligned}
W=\left\{\left(\sigma_{i j}, \tau_{k l}\right) \in R \mid \tau_{k l}\right. & =\mu_{k}, \text { para }(k, l) \in J_{4}, \\
& \tau_{m / 2, l}=\mu_{m / 2}, \text { para } l \in J_{5}, \\
& \text { e algum } \mu_{k} \in K^{*}, \\
& \text { em que } \left.\mu_{\frac{m}{2}}=1 \text { se } 2 \mid m\right\},
\end{aligned}
$$

que é obtido a partir de $R$ removendo os quadrados nas relações que correspondem a $J_{4}$ e $J_{5}$.

Será verificado que $W \leq \operatorname{ker}(\psi)$. De fato, dado $\left(\sigma_{i j}, \tau_{k l}\right) \in W$, seja $\lambda: D_{2 m} \rightarrow K^{*}$ a aplicação definida por

$$
\begin{gathered}
\lambda\left(a^{i} b\right)=\lambda(1)=1, \text { para } 0 \leq i \leq m-1, \\
\lambda\left(a^{i}\right)=\frac{1}{\lambda\left(a^{m-i}\right)}=\mu_{i}, \text { para } 1 \leq i \leq\lfloor(m-1) / 2\rfloor
\end{gathered}
$$

e $\lambda\left(a^{\frac{m}{2}}\right)=1$ se $2 \mid m$. Então, resulta das definições de $R$ e $W$ que

$$
\begin{gathered}
\sigma_{1 j}=\frac{\tau_{10} \tau_{j 1}}{\tau_{1+j, 0}}=\frac{\mu_{1} \mu_{j}}{\mu_{1+j}}=\lambda(a) \lambda\left(a^{j}\right) / \lambda\left(a^{1+j}\right), \text { para } j \in J_{3}, \\
\sigma_{1 j}=\sigma_{1, m-j-1}=\lambda(a) \lambda\left(a^{m-j-1}\right) / \lambda\left(a^{m-j}\right)=\lambda(a) \lambda\left(a^{j}\right) / \lambda\left(a^{j+1}\right), \text { para } j \in J_{2}, \\
\sigma_{i j}=\frac{\sigma_{1 i} \ldots \sigma_{1, i+j-1}}{\sigma_{11} \ldots \sigma_{1, j-1}} \\
=\left(\frac{\lambda(a) \lambda\left(a^{i}\right)}{\lambda\left(a^{i+1}\right)} \frac{\lambda(a) \lambda\left(a^{i+1}\right)}{\lambda\left(a^{i+2}\right)} \ldots \frac{\lambda(a) \lambda\left(a^{i+j-1}\right)}{\lambda\left(a^{i+j}\right)}\right)\left(\frac{\lambda\left(a^{2}\right)}{\lambda(a) \lambda(a)} \frac{\lambda\left(a^{3}\right)}{\lambda(a) \lambda\left(a^{2}\right)} \cdots \frac{\lambda\left(a^{j}\right)}{\lambda(a) \lambda\left(a^{j-1}\right)}\right) \\
=\frac{\lambda^{j}(a) \lambda\left(a^{i}\right)}{\lambda\left(a^{i+j}\right)} \frac{\lambda\left(a^{j}\right)}{\lambda^{j}(a)} \\
=\frac{\lambda\left(a^{i}\right) \lambda\left(a^{j}\right)}{\lambda\left(a^{i+j}\right)}, \operatorname{para}(i, j) \in J_{1},
\end{gathered}
$$

e

$$
\tau_{k l}=\mu_{k}=\lambda\left(a^{k}\right) \lambda\left(a^{l} b\right) / \lambda\left(a^{k+l} b\right), \text { para }(k, l) \in J_{4} \cup\left(\{m / 2\} \times J_{5}\right) .
$$

Consequentemente, $W \triangleleft \operatorname{ker}(\psi)$.

Será construído um epimorfismo cujo núcleo é $W$. Como $\sum_{i=1}^{5}\left|J_{i}\right|=d_{m}$, e $\left|(k, l) \in J_{4}, l \neq 0\right|=$ $\left|J_{4}\right|-\lfloor(m-1) / 2\rfloor$, pode-se definir um homomorfismo $\Lambda:\left(K^{*}\right)^{d_{m}} \rightarrow\left(K^{*}\right)^{d_{m}-\left\lfloor\frac{m-1}{2}\right\rfloor}$ levando $\left(\sigma_{i j}, \tau_{k l}\right)$ em

$$
\left(\left(\frac{\sigma_{1 j} \tau_{j+1,0}}{\tau_{10} \tau_{j 1}}\right)_{j \in J_{3}},\left(\frac{\sigma_{1 j}}{\sigma_{1, m-j-1}}\right)_{j \in J_{2}},\left(\frac{\sigma_{i j} \sigma_{11} \ldots \sigma_{1, j-1}}{\sigma_{1 i} \ldots \sigma_{1, i+j-1}}\right)_{(i, j) \in J_{1}},\left(\frac{\tau_{k l}}{\tau_{k 0}}\right)_{(k, l) \in J_{4}, l \neq 0},\left(\tau_{\frac{m}{2}, l}\right)_{l \in J_{5}}\right) .
$$

Então por construção $W=\operatorname{ker}(\Lambda)$, e $\Lambda$ é um epimorfismo, pois para todo

$$
z=\left(\left(u_{i}\right)_{i \in J_{3}},\left(v_{i}\right)_{i \in J_{2}},\left(w_{i, j}\right)_{(i, j) \in J_{1}},\left(x_{i, j}\right)_{(i, j) \in J_{4}, j \neq 0},\left(y_{i}\right)_{i \in J_{5}}\right) \in\left(K^{*}\right)^{d_{m}-\left\lfloor\frac{m-1}{2}\right\rfloor},
$$

tem-se $\Lambda\left(\sigma_{i j}, \tau_{k l}\right)=z$, em que

$$
\begin{gathered}
\tau_{k 0}=1 \text { para }(k, 0) \in J_{4}, \\
\tau_{k l}=x_{k l} \text { para }(k, l) \in J_{4}, l \neq 0, \\
\tau_{\frac{m}{2} l}=y_{l}, \quad \text { para } l \in J_{5}, \\
\sigma_{1 j}=\frac{u_{j} \tau_{10} \tau_{j 1}}{\tau_{j+1,0}}, \quad \text { para } j \in J_{3},
\end{gathered}
$$




$$
\sigma_{1 j}=v_{j} \sigma_{1, m-j-1}, \quad \text { para } j \in J_{2}
$$

e

$$
\sigma_{i j}=\frac{w_{i j} \sigma_{1 i} \ldots \sigma_{1, i+j-1}}{\sigma_{11} \ldots \sigma_{1, j-1}}, \quad \text { para }(i, j) \in J_{1}
$$

Dado $x \in \operatorname{ker}(\psi) \subseteq R$, tem-se $\Lambda(x)=\left(1, \ldots, 1, \varepsilon_{1}, \ldots, \varepsilon_{t}\right)$, em que a quantidade de elementos 1 é dada por $\left|J_{3}\right|+\left|J_{2}\right|+\left|J_{1}\right|$ e $\varepsilon_{i}^{2}=1$, para $1 \leq i \leq t=\left|J_{4}\right|-\left\lfloor\frac{m-1}{2}\right\rfloor+\left|J_{5}\right|$. Em outras palavras, $\Lambda(\operatorname{ker}(\psi)) \triangleleft C_{2}^{d_{m}-\left\lfloor\frac{m-1}{2}\right\rfloor}$, em que $C_{2}=\{1,-1\}$.

Portanto, pelo Teorema 1.5.12,

$$
p M_{D_{2 m} \times D_{2 m}}\left(D_{2 m}\right) \simeq \frac{\left(K^{*}\right)^{d_{m}}}{\operatorname{ker}(\psi)} \simeq \frac{\frac{\left(K^{*}\right)^{d_{m}}}{\operatorname{ker}(\Lambda)}}{\frac{\operatorname{ker}(\psi)}{\operatorname{ker}(\Lambda)}} \simeq \frac{\left(K^{*}\right)^{d_{m}-\left\lfloor\frac{m-1}{2}\right\rfloor}}{\Lambda(\operatorname{ker}(\psi))} \simeq\left(K^{*}\right)^{d_{m}-\left\lfloor\frac{m-1}{2}\right\rfloor}
$$

em que o último isomorfismo resulta de (4.1).

\subsection{Produto Direto de Grupos Cíclicos}

Recorde-se que, dado um grupo $G$, foi definida uma ação do semigrupo $\mathcal{T}$ em $G \times G$ por meio das transformações indicadas em (1.5) e que $S_{3} \simeq\langle u, v\rangle$.

Denote por $G H$ o produto direto dos grupos $G$ e $H$, e por $\left(g_{1} h_{1}, g_{2} h_{2}\right)$ um elemento arbitrário do produto cartesiano $G H \times G H$, em que $g_{1}, g_{2} \in G$ e $h_{1}, h_{2} \in H$.

Lema 4.2.1. Para quaisquer grupos $G$ e $H$, qualquer $x \in S_{3}$, e elementos arbitrários $g_{i}, g_{i}^{\prime} \in$ $G, h_{i}, h_{i}^{\prime} \in H$,

$$
\left(g_{1}^{\prime} h_{1}^{\prime}, g_{2}^{\prime} h_{2}^{\prime}\right)=x\left(g_{1} h_{1}, g_{2} h_{2}\right) \Leftrightarrow\left(g_{1}^{\prime}, g_{2}^{\prime}\right)=x\left(g_{1}, g_{2}\right) e\left(h_{1}^{\prime}, h_{2}^{\prime}\right)=x\left(h_{1}, h_{2}\right) .
$$

Demonstração. É suficiente mostrar (4.17) para os geradores $u$ e $v$ de $S_{3}$. Como no produto direto $G H$ os elementos de $G$ comutam com os de $H$, resulta que

$$
u\left(g_{1} h_{1}, g_{2} h_{2}\right)=\left(\left(g_{1} h_{1}\right)\left(g_{2} h_{2}\right),\left(g_{2} h_{2}\right)^{-1}\right)=\left(\left(g_{1} g_{2}\right)\left(h_{1} h_{2}\right), g_{2}^{-1} h_{2}^{-1}\right)
$$

e consequentemente

$$
\left(g_{1}^{\prime} h_{1}^{\prime}, g_{2}^{\prime} h_{2}^{\prime}\right)=u\left(g_{1} h_{1}, g_{2} h_{2}\right) \Leftrightarrow g_{1}^{\prime}=g_{1} g_{2} \text { e } g_{2}^{\prime}=g_{2}^{-1} \text { e } h_{1}^{\prime}=h_{1} h_{2} \text { e } h_{2}^{\prime}=h_{2}^{-1} .
$$

Analogamente, como

$$
v\left(g_{1} h_{1}, g_{2} h_{2}\right)=\left(\left(g_{2} h_{2}\right)^{-1},\left(g_{1} h_{1}\right)^{-1}\right)=\left(g_{2}^{-1} h_{2}^{-1}, g_{1}^{-1} h_{1}^{-1}\right),
$$

resulta que

$$
\left(g_{1}^{\prime} h_{1}^{\prime}, g_{2}^{\prime} h_{2}^{\prime}\right)=v\left(g_{1} h_{1}, g_{2} h_{2}\right) \Leftrightarrow g_{1}^{\prime}=g_{2}^{-1} \text { e } g_{2}^{\prime}=h_{2}^{-1} \text { e } h_{1}^{\prime}=g_{1}^{-1} \text { e } h_{2}^{\prime}=h_{1}^{-1} .
$$

Agora o resultado é imediato.

Dado um grupo $G$, seja $T_{G}$ um conjunto contendo exatamente um representante de cada órbita da ação de $S_{3}$ em $G \times G$, e $S_{G}$ o subconjunto de $T_{G}$ formado pelos elementos que correspondem às órbitas efetivas. Nesse texto, $T_{G}$ sempre será escolhido de modo que $T_{G} \backslash S_{G}=\{1\} \times G$. Isso é possível porque por (1.6) as órbitas não efetivas são da forma $\left\{(1, x),\left(x, x^{-1}\right),\left(x^{-1}, 1\right)\right\}$, com $x \in G$. No caso particular em que $G=C_{m}$, será conveniente aplicar o Lema 4.0.10 e escolher

$$
\begin{aligned}
S_{C_{m}} & =\left\{\left(a^{i}, a^{j}\right) \mid 1 \leq i<m / 3 \text { e } i \leq j \leq m-2 i-1\right\} \\
& \cup\left\{\left(a^{i}, a^{j}\right) \mid i=j=m / 3 \in \mathbb{Z}\right\},
\end{aligned}
$$


se $m \geq 3$, e $S_{C_{m}}=\emptyset$, se $m \in\{1,2\}$.

Denote $o_{k}(G)=\{g \in G \mid \operatorname{ord}(g)=k\}$ e $G^{*}=G \backslash\{1\}$. Se $G$ tem elementos de ordem maior do que 2, escolha $X(G) \subseteq G$ tal que $G \backslash o_{1}(G) \cup o_{2}(G)=X(G) \cup X(G)^{-1}$ e $X(G) \cap X(G)^{-1}=\emptyset$. Caso contrário, defina $X(G)=\emptyset$.

Se $|G|$ é par, considere para cada $e \in o_{2}(G)$ um conjunto $Y(e) \subsetneq G$ tal que $G=Y(e) \cup e Y(e)$ e $Y(e) \cap e Y(e)=\emptyset$.

Exemplo 4.2.2. Se $G=C_{m}=\left\langle a \mid a^{m}=1\right\rangle$ e $m \geq 3$, escolha

$$
X\left(C_{m}\right)=\left\{a, a^{2}, \ldots, a^{\left\lfloor\frac{m-1}{2}\right\rfloor}\right\} .
$$

Agora, se $m$ é par, o único elemento de ordem 2 é $e=a^{\frac{m}{2}}$, e uma escolha natural é

$$
Y\left(a^{\frac{m}{2}}\right)=\left\{1, a, \ldots, a^{\frac{m}{2}-1}\right\} .
$$

Para $G=\mathbb{Z}=\langle a\rangle$, escolha $X(\mathbb{Z})=\mathbb{N}=\left\{a^{i} \mid i>0\right\}$.

O próximo lema fornecerá uma lista de representantes das órbitas efetivas de um produto direto de grupos. O caso particular do produto de grupos cíclicos finitos é especificado no Exemplo 4.2.4, que na verdade foi o que serviu de inspiração para a versão mais geral desse resultado.

Lema 4.2.3. Dados grupos $G$ e $H$ arbitrários, fixe $S_{G}, S_{H}, T_{G}, X(G)$ e $Y\left(\right.$ e) (se e $\in G_{(2)}$ ) como acima. Então o conjunto

$$
\begin{aligned}
S_{G} & \cup\left(X(G) \times G H^{*}\right) \\
& \cup \bigcup_{e \in o_{2}(G)}\{e\} \times Y(e) H^{*} \\
& \cup\left\{\left(g_{1} h_{1}, g_{2} h_{2}\right) \mid g_{1}, g_{2} \in G \text { e }\left(h_{1}, h_{2}\right) \in S_{H}, \text { em que }\left(h_{1}, h_{2}\right) \notin H_{(3)} \times H_{(3)} \text { ou } h_{1} \neq h_{2}\right\} \\
& \cup\left\{\left(g_{1} h, g_{2} h\right) \mid\left(g_{1}, g_{2}\right) \in T_{G} \cup v\left(S_{G}\right),(h, h) \in S_{H} \cap\left(H_{(3)} \times H_{(3)}\right)\right\}
\end{aligned}
$$

contém exatamente um representante de cada órbita efetiva de $S_{3}$ em $G H \times G H$.

Demonstração. Considere a seguinte decomposição:

$$
G H \times G H=(G \times G) \cup\left(G H^{*} \times G\right) \cup\left(G \times G H^{*}\right) \cup\left(G H^{*} \times G H^{*}\right) .
$$

Por (1.6), a órbita de qualquer $\left(g_{1} h_{1}, g_{2} h_{2}\right) \in G H \times G H$ é da forma

$$
\begin{aligned}
S_{3}\left(g_{1} h_{1}, g_{2} h_{2}\right)=\{ & \left(g_{1} h_{1}, g_{2} h_{2}\right),\left(g_{2}^{-1} g_{1}^{-1} h_{2}^{-1} h_{1}^{-1}, g_{1} h_{1}\right),\left(g_{2} h_{2}, g_{2}^{-1} g_{1}^{-1} h_{2}^{-1} h_{1}^{-1}\right), \\
& \left.\left(g_{2}^{-1} h_{2}^{-1}, g_{1}^{-1} h_{1}^{-1}\right),\left(g_{1}^{-1} h_{1}^{-1}, g_{1} g_{2} h_{1} h_{2}\right),\left(g_{1} g_{2} h_{1} h_{2}, g_{2}^{-1} h_{2}^{-1}\right)\right\} .
\end{aligned}
$$

Em particular, se $h_{1}=h_{2}=1$ então $S_{3}\left(g_{1}, g_{2}\right) \subseteq G \times G$. Consequentemente $S_{G}$ contém exatamente um representante de cada uma dessas órbitas efetivas da ação de $S_{3}$ em $G H \times G H$. Agora, considere uma das $S_{3}$-órbitas restantes, isto é, a órbita de um par de $(G H \times G H) \backslash(G \times G)$.

- Se a órbita contém algum par $\left(g_{1} h_{1}, g_{2}\right) \in G H^{*} \times G$, ela também tem um representante $\left(g_{2}^{-1}, g_{1}^{-1} h_{1}^{-1}\right) \in G \times G H^{*}$. Portanto, pode-se escolher pares de $G \times G H^{*}$ em vez de pares de $G H^{*} \times G$.

- Se a órbita é efetiva e contém um representante $\left(g_{1}, g_{2} h_{2}\right) \in G \times G H^{*}$, então $\left(g_{1}^{-1}, g_{1} g_{2} h_{2}\right)$ também está em $S_{3}\left(g_{1}, g_{2} h_{2}\right)$. Se ord $\left(g_{1}\right) \neq 1,2$, então $g_{1} \neq g_{1}^{-1}$ e é suficiente escolher um destes pares distintos. Seja $\left(g_{1}, g_{2} h_{2}\right)$ aquele tal que $g_{1} \in X$. Por outro lado, se $\operatorname{ord}\left(g_{1}\right)=$ 2 , então $g_{1}^{-1}=g_{1} \neq 1$ e $\left(g_{1}, g_{1} g_{2} h_{2}\right) \neq\left(g_{1}, g_{2} h_{2}\right)$. Neste caso, para evitar que haja dois representantes para a mesma $S_{3}$-órbita, suponha que $g_{2}$ pertence a $Y\left(g_{1}\right)$.

- Finalmente, suponha que a órbita é efetiva e contém algum $\left(g_{1} h_{1}, g_{2} h_{2}\right) \in G H^{*} \times G H^{*}$. Se ela intersecta $G \times G H^{*}$, escolha um representante como no caso anterior. Do contrário, $h_{1} h_{2} \neq 1$ 
e $S_{3}\left(h_{1}, h_{2}\right)$ é uma órbita efetiva (veja $\left.(1.7)\right)$. Consequentemente, $\left(h_{1}, h_{2}\right)=x\left(h_{1}^{\prime}, h_{2}^{\prime}\right)$ para algum $x \in S_{3}$ e algum $\left(h_{1}^{\prime}, h_{2}^{\prime}\right) \in S_{H}$. Pelo Lema 4.2 .1 , se $\left(g_{1}^{\prime}, g_{2}^{\prime}\right)=x^{-1}\left(g_{1}, g_{2}\right)$, então $\left(g_{1} h_{1}, g_{2} h_{2}\right)=x\left(g_{1}^{\prime} h_{1}^{\prime}, g_{2}^{\prime} h_{2}^{\prime}\right)$. Portanto, é suficiente considerar representantes no conjunto

$$
(G \times G) S_{H}=\left\{\left(g_{1} h_{1}, g_{2} h_{2}\right) \mid\left(g_{1}, g_{2}\right) \in G \times G,\left(h_{1}, h_{2}\right) \in S_{H}\right\} .
$$

Observe que, pelo Lema 4.2 .1 , dois pares $\left(g_{1}^{\prime} h_{1}^{\prime}, g_{2}^{\prime} h_{2}^{\prime}\right)$ e $\left(g_{1}^{\prime \prime} h_{1}^{\prime \prime}, g_{2}^{\prime \prime} h_{2}^{\prime \prime}\right)$ deste conjunto estão na mesma órbita se, e somente se, existe $y \in S_{3}$ tal que $\left(g_{1}^{\prime}, g_{2}^{\prime}\right)=y\left(g_{1}^{\prime \prime}, g_{2}^{\prime \prime}\right)$ e $\left(h_{1}^{\prime}, h_{2}^{\prime}\right)=y\left(h_{1}^{\prime \prime}, h_{2}^{\prime \prime}\right)$. Nesse caso, como $S_{H}$ contém apenas um par de cada órbita efetiva, resulta que $\left(h_{1}^{\prime}, h_{2}^{\prime}\right)=$ $\left(h_{1}^{\prime \prime}, h_{2}^{\prime \prime}\right)$. Uma inspeção dos elementos da órbita efetiva $S_{3}\left(h_{1}^{\prime \prime}, h_{2}^{\prime \prime}\right)$, dados por (1.6), deixa claro que

$$
\left(h_{1}^{\prime \prime}, h_{2}^{\prime \prime}\right)=y\left(h_{1}^{\prime \prime}, h_{2}^{\prime \prime}\right) \Leftrightarrow y \in\left\{1, u v,(u v)^{2}\right\}, h_{1}^{\prime \prime}=h_{2}^{\prime \prime} \mathrm{e} \operatorname{ord}\left(h_{1}^{\prime \prime}\right)=3 .
$$

Suponha que $(h, h) \in S_{H} \cap\left(H_{(3)} \times H_{(3)}\right)$.

Se $S_{3}\left(g_{1}, g_{2}\right)$ é efetiva então $\left(g_{1}, g_{2}\right)=z\left(g_{1}^{\prime}, g_{2}^{\prime}\right)$, para algum $\left(g_{1}^{\prime}, g_{2}^{\prime}\right) \in S_{G}$ e algum $z \in S_{3}$. Se $z \in\left\{1, u v,(u v)^{2}\right\}$ então, por $(4.21),(h, h)=z(h, h)$ e consequentemente $\left(g_{1} h, g_{2} h\right)=$ $z\left(g_{1}^{\prime} h, g_{2}^{\prime} h\right)$ pelo Lema 4.2.1. Caso contrário, $z=v w$, com $w \in\left\{1, u v,(u v)^{2}\right\}$ e

$$
\left(g_{1}, g_{2}\right)=v w\left(g_{1}^{\prime}, g_{2}^{\prime}\right)=w^{-1} v\left(g_{1}^{\prime}, g_{2}^{\prime}\right)=w^{-1}\left(g_{2}^{-1}, g_{1}^{-1}\right)
$$

Por (4.21) e pelo Lema 4.2.1, resulta que $\left(g_{1} h, g_{2} h\right)=w^{-1}\left(g_{2}^{-1} h, g_{1}^{-1} h\right)$, em que $\left(g_{2}^{-1}, g_{1}^{-1}\right)=$ $v\left(g_{1}^{\prime}, g_{2}^{\prime}\right) \in v\left(S_{G}\right)$. Assim, para qualquer órbita $S_{3}\left(g_{1} h, g_{2} h\right)$, tal que $\left(g_{1}, g_{2}\right)$ é efetiva e $(h, h) \in$ $S_{H} \cap\left(H_{(3)} \times H_{(3)}\right)$, pode ser assumido que $\left(g_{1}, g_{2}\right) \in S_{G} \cup v\left(S_{G}\right)$.

Por outro lado, se $S_{3}\left(g_{1}, g_{2}\right)$ não é efetiva então $\left(g_{1}, g_{2}\right)=z\left(g_{1}^{\prime}, g_{2}^{\prime}\right)$, para algum $\left(g_{1}^{\prime}, g_{2}^{\prime}\right) \in$ $T_{G} \backslash S_{G}$ e algum $z \in S_{3}$. Considerando que $v\left(S_{3}\left(g_{1}, g_{2}\right)\right)=S_{3}\left(g_{1}, g_{2}\right)$, é possível escolher $z \in\left\{1, u v,(u v)^{2}\right\}$ e obter $\left(g_{1} h, g_{2} h\right)=z\left(g_{1}^{\prime} h, g_{2}^{\prime} h\right)$ por $(4.21)$, para qualquer $(h, h) \in S_{H} \cap$ $\left(H_{(3)} \times H_{(3)}\right)$.

Como consequência do lema anterior, pode-se obter uma lista de representantes para as órbitas efetivas dos produtos de grupos cíclicos finitos. O Apêndice B mostra como são as órbitas efetivas em dois exemplos concretos, e pode ser consultado para auxiliar na visualização do resultado.

Exemplo 4.2.4. Seja $G=C_{m} \times C_{n}=\left\langle a, b \mid a^{m}=b^{n}=a^{-1} b^{-1} a b=1\right\rangle$, para alguns inteiros $m$ e $n$. Utilizando as notações estabelecidas em (4.18), (4.19) e (4.20), defina

$$
\begin{aligned}
S_{C_{m} \times C_{n}} & =S_{C_{m}} \\
& \cup X\left(C_{m}\right) \times C_{m} C_{n}^{*} \\
& \cup\left\{a^{m / 2}\right\} \times Y\left(C_{m}\right) C_{n}^{*} \\
& \cup\left(C_{m} \times C_{m}\right)\left(S_{C_{n}} \backslash\left\{\left(b^{\frac{n}{3}}, b^{\frac{n}{3}}\right)\right\}\right) \\
& \cup\left(\left(\{1\} \times C_{m}\right) \cup S_{C_{m}} \cup v\left(S_{C_{m}}\right)\right)\left\{\left(b^{\frac{n}{3}}, b^{\frac{n}{3}}\right)\right\} .
\end{aligned}
$$

em que $Y\left(C_{m}\right)=Y\left(a^{\frac{m}{2}}\right)$ se $2 \mid m, Y\left(C_{m}\right)=\emptyset$ se $2 \nmid m$ e $\left\{\left(b^{\frac{n}{3}}, b^{\frac{n}{3}}\right)\right\}=\emptyset$ se $3 \nmid n$.

Nessas circunstâncias, $S_{C_{m} \times C_{n}}$ contém precisamente um representante de cada $S_{3}$-órbita efetiva. Em particular, qualquer $\sigma \in \mathrm{pm}_{G \times G}^{\prime}(G)$ fica completamente determinado por seus valores nos elementos de $S_{C_{m} \times C_{n}}$, e estes valores podem ser escolhidos arbitrariamente em $K^{*}$.

Proposição 4.2.5. Seja $G=C_{m} \times C_{n}$. Se $\sigma \in \operatorname{pm}_{G \times G}^{\prime}(G)$ e $\sigma \sim 1$ então $\sigma$ é unicamente deter- 
minado por seus valores nos pares

$$
\begin{aligned}
\left(a, a^{k} b^{l}\right), \text { em que } 0 & \leq k \leq m-1,1 \leq l \leq\lfloor(n-1) / 2\rfloor \\
\left(a, a^{k} b^{n / 2}\right), \text { em que } 0 & \leq k \leq\lfloor(m-1) / 2\rfloor \text { (somente se } n \text { é par }), \\
\left(a^{i}, b\right), \text { em que } 2 & \leq i \leq\lfloor m / 2\rfloor, \\
\left(b, b^{l}\right), \text { em que } 1 & \leq l \leq\lfloor(n-1) / 2\rfloor \text { (somente se } n \geq 3),
\end{aligned}
$$

e esses valores podem ser escolhidos arbitrariamente em $K^{*}$.

Demonstração. Assuma que $G=C_{m} \times C_{n}$ é gerado por $a$ e $b$ como no Exemplo 4.2.4. Como $\sigma \sim 1$, existe uma aplicação $\rho: G \rightarrow K^{*}$ satisfazendo (1.11) e (1.12) tal que $\sigma(x, y)=\rho(x) \rho(y) / \rho(x y)$, para quaisquer $x, y \in G$. Observe que, por (4.14) e pelo Exemplo 4.2.4, o valor de $\sigma$ em qualquer $\left(a^{i}, a^{k}\right) \in S_{C_{m}}$ é determinado por seus valores em alguns elementos de

$$
\begin{aligned}
B_{1}= & \left(X\left(C_{m}\right) \times C_{m} C_{n}^{*}\right) \cup\left(\left\{a^{m / 2}\right\} \times Y\left(C_{m}\right) C_{n}^{*}\right) \\
= & \left\{\left(a^{i}, a^{k} b^{l}\right) \mid 1 \leq i \leq\lfloor(m-1) / 2\rfloor, 0 \leq k \leq m-1 \text { e } 1 \leq l \leq n-1\right\} \\
& \cup\left\{\left(a^{m / 2}, a^{k} b^{l}\right) \mid 0 \leq k \leq m / 2-1 \text { e } 1 \leq l \leq n-1\right\} .
\end{aligned}
$$

Por (4.13), se $i, k \geq 1$ então

$$
\sigma\left(a^{i}, a^{k} b^{l}\right)=\frac{\sigma_{i+k}\left(a, b^{l}\right) \sigma\left(a^{i}, b^{l}\right)}{\sigma_{i}\left(a, b^{l}\right) \sigma_{k}\left(a, b^{l}\right)}=\frac{\sigma\left(a, a^{i} b^{l}\right) \ldots \sigma\left(a, a^{i+k-1} b^{l}\right) \sigma\left(a^{i}, b^{l}\right)}{\sigma\left(a, b^{l}\right) \ldots \sigma\left(a, a^{k-1} b^{l}\right)} .
$$

Assim, para $i \geq 2$, o valor de $\sigma\left(a^{i}, a^{k} b^{l}\right)$ é determinado pelos valores $\sigma\left(a, a^{k^{\prime}} b^{l^{\prime}}\right)$ e $\sigma\left(a^{i^{\prime}}, b^{l^{\prime}}\right)$ em que $1 \leq k^{\prime} \leq m-1,1 \leq l^{\prime} \leq n-1$ e $1 \leq i^{\prime} \leq\lfloor m / 2\rfloor$.

Agora serão considerados os valores $\sigma\left(a, a^{k} b^{l}\right)$ e $\sigma\left(a^{i}, b^{l}\right)$. No primeiro caso,

$$
\sigma\left(a^{i}, a^{k} b^{l}\right)=\frac{\rho\left(a^{i}\right) \rho\left(a^{k} b^{l}\right)}{\rho\left(a^{i+k} b^{l}\right)}=\frac{\rho\left(a^{i}\right) \rho\left(a^{m-i-k} b^{n-l}\right)}{\rho\left(a^{m-k} b^{n-l}\right)}=\sigma\left(a^{i}, a^{m-i-k} b^{n-l}\right),
$$

então, em particular, para $i=1$ tem-se $\sigma\left(a, a^{k} b^{l}\right)=\sigma\left(a, a^{m-1-k} b^{n-l}\right)$. Assumindo que $l \leq n-l$, tem-se $1 \leq l \leq n / 2$. Se, além disso, $n$ é par e $l=n / 2$ então $\sigma\left(a, a^{k} b^{n / 2}\right)=\sigma\left(a, a^{m-1-k} b^{n / 2}\right)$, e pode-se assumir que $k \leq m-1-k$, isto é, $k \leq\lfloor(m-1) / 2\rfloor$.

Com relação aos valores $\sigma\left(a^{i}, b^{l}\right)$, tem-se

$$
\frac{\sigma\left(a^{i}, b^{l}\right)}{\sigma_{i}\left(a, b^{l}\right)}=\frac{\rho\left(a^{i}\right) \rho\left(b^{l}\right)}{\rho\left(a^{i} b^{l}\right)} \frac{\rho\left(a^{i} b^{l}\right)}{\rho^{i}(a) \rho\left(b^{l}\right)}=\frac{\rho\left(a^{i}\right)}{\rho^{i}(a)},
$$

para todo $l$ e $i \geq 1$, resulta que se $i, l \geq 2$ então

$$
\sigma\left(a^{i}, b^{l}\right)=\sigma\left(a^{i}, b\right) \frac{\sigma_{i}\left(a, b^{l}\right)}{\sigma_{i}(a, b)} .
$$

Consequentemente, os valores de $\sigma$ nos elementos de $B_{1}$ são determinados por

$$
\begin{gathered}
\sigma\left(a, a^{k} b\right), \sigma\left(a, a^{k} b^{2}\right), \ldots, \sigma\left(a, a^{k} b^{\lfloor(n-1) / 2\rfloor}\right), \text { em que } 0 \leq k \leq m-1, \\
\sigma\left(a^{i}, b\right), \text { em que } 2 \leq i \leq\lfloor m / 2\rfloor,
\end{gathered}
$$

e (se $n$ é par)

$$
\sigma\left(a, a^{k} b^{n / 2}\right), \text { em que } 0 \leq k \leq\lfloor(m-1) / 2\rfloor .
$$

Aplicando-se o Lema 4.0.10 a $C_{n}=\langle b\rangle$, resulta que o conjunto

$$
B_{2}=\left(C_{m} \times C_{m}\right)\left(S_{C_{n}} \backslash\left\{\left(b^{\frac{n}{3}}, b^{\frac{n}{3}}\right)\right\}\right) \cup\left(\left(\{1\} \times C_{m}\right) \cup S_{C_{m}} \cup v\left(S_{C_{m}}\right)\right)\left\{\left(b^{\frac{n}{3}}, b^{\frac{n}{3}}\right)\right\}
$$


do Exemplo 4.2.4 não é vazio se $n \geq 3$, em que $\left\{\left(b^{\frac{n}{3}}, b^{\frac{n}{3}}\right)\right\}=\emptyset$ se $3 \nmid n$. Além disso, para $i, j, k, l \in \mathbb{Z}$,

$$
\begin{aligned}
\sigma\left(a^{i} b^{j}, a^{k} b^{l}\right) & =\frac{\rho\left(a^{i} b^{j}\right) \rho\left(a^{k} b^{l}\right)}{\rho\left(a^{i+k} b^{j+l}\right)} \\
& =\frac{\rho\left(a^{i}\right) \rho\left(b^{j}\right) \rho\left(a^{k} b^{l}\right)}{\sigma\left(a^{i}, b^{j}\right) \rho\left(a^{i+k} b^{j+l}\right)} \\
& =\frac{\sigma\left(a^{i}, a^{k} b^{l}\right) \rho\left(a^{i+k} b^{l}\right) \rho\left(b^{j}\right)}{\sigma\left(a^{i}, b^{j}\right) \rho\left(a^{i+k} b^{j+l}\right)} \\
& =\frac{\sigma\left(a^{i}, a^{k} b^{l}\right) \rho\left(a^{i+k}\right) \rho\left(b^{l}\right) \rho\left(b^{j}\right)}{\sigma\left(a^{i}, b^{j}\right) \sigma\left(a^{i+k}, b^{l}\right) \rho\left(a^{i+k} b^{j+l}\right)} \\
& =\sigma\left(b^{j}, b^{l}\right) \frac{\sigma\left(a^{i}, a^{k} b^{l}\right) \sigma\left(a^{i+k}, b^{j+l}\right)}{\sigma\left(a^{i}, b^{j}\right) \sigma\left(a^{i+k}, b^{l}\right)} .
\end{aligned}
$$

Portanto, os valores do conjunto fator parcial $\sigma$ em qualquer $\left(a^{i} b^{j}, a^{k} b^{l}\right) \in B_{2}$ é determinado por aqueles que já foram especificados em (4.29), (4.30) e (4.31), juntamente com seus valores $\sigma\left(b^{j}, b^{l}\right)$ em que $\left(b^{j}, b^{l}\right) \in S_{C_{n}}$. Aplicando o Lema 4.0.12 ao grupo $C_{n}$, conclui-se que esses últimos são determinados por

$$
\sigma\left(b, b^{l}\right), \text { em que } 1 \leq l \leq\lfloor(n-1) / 2\rfloor,
$$

por meio de

$$
\sigma\left(b^{j}, b^{l}\right)=\frac{\pi_{j+l}}{\pi_{j} \pi_{l}}=\frac{\sigma_{j+l-1}(b, b)}{\sigma_{j-1}(b, b) \sigma_{l-1}(b, b)},
$$

em que $\pi_{j}=\pi_{j}(b)$ é dado por (4.8), e

$$
\sigma\left(b, b^{l}\right)=\sigma\left(b, b^{n-l-1}\right) .
$$

Reciprocamente, denote por $I$ a lista de pares indicados em (4.22)-(4.25) e fixe uma família $(\nu(x, y))_{(x, y) \in I}$ de elementos de $K^{*}$. No que vem a seguir, a intenção é mostrar que esses valores determinam um conjunto fator parcial $\sigma \sim 1$. Pelo Corolário 1.5.11 (ii), se for construída uma aplicação $\rho: G \rightarrow K^{*}$ que satisfaça (1.11) e (1.12) tal que

$$
\sigma(x, y)=\frac{\rho(x) \rho(y)}{\rho(x y)}, \text { para qualquer }(x, y) \in I,
$$

então a igualdade (4.34) definirá um conjunto fator parcial $\sigma \in p m_{G \times G}^{\prime}(G)$ tal que $\sigma \sim 1$.

Seja $\nu_{0}(x, y)=1$ e $\nu_{j}(x, y)=\nu(x, y) \nu(x, x y) \ldots \nu\left(x, x^{j-1} y\right)$, em que

- $(x, y)=\left(a, b^{l}\right), 1 \leq j \leq m$ e $1 \leq l \leq\lfloor(n-1) / 2\rfloor$; ou

- $(x, y)=\left(a, b^{n / 2}\right), 1 \leq j \leq\lfloor(m+1) / 2\rfloor$ (somente se $n$ é par); ou

- $(x, y)=(b, b)$ e $1 \leq j \leq\lfloor(m+1) / 2\rfloor$ (somente se $n \geq 3)$.

A intenção é definir $\rho(b), \ldots, \rho\left(b^{\left.\frac{n+1}{2}\right\rfloor}\right)$ de tal modo que $\rho$ satisfaça (1.12) e

$$
\nu_{j}(b, b)=\frac{\rho^{j+1}(b)}{\rho\left(b^{j+1}\right)}
$$

para $j=1, \ldots,\lfloor(n-1) / 2\rfloor$ (isso já é verdadeiro para $j=0$ ), e os valores de $\rho$ nas demais potências de $b$ (se $n \geq 3$ ) serão definidos por meio de (1.12). Como $n=\left\lfloor\frac{n}{2}\right\rfloor+\left\lfloor\frac{n+1}{2}\right\rfloor$, isso só é possível se

$\rho^{n}(b)=\rho^{\left\lfloor\frac{n}{2}\right\rfloor}(b) \rho^{\left\lfloor\frac{n+1}{2}\right\rfloor}(b)=\left(\nu_{\left\lfloor\frac{n}{2}\right\rfloor-1}(b, b) \rho\left(b^{\left\lfloor\frac{n}{2}\right\rfloor}\right)\right)\left(\nu_{\left\lfloor\frac{n-1}{2}\right\rfloor}(b, b) \rho\left(b^{\left\lfloor\frac{n+1}{2}\right\rfloor}\right)\right)=\nu_{\left\lfloor\frac{n}{2}\right\rfloor-1}(b, b) \nu_{\left\lfloor\frac{n-1}{2}\right\rfloor}(b, b)$.

Então defina $\rho(b)=1 / \rho\left(b^{n-1}\right)=\omega_{2}$, em que $\omega_{2}^{n}=\nu_{\left\lfloor\frac{n}{2}\right\rfloor-1}(b, b) \nu_{\left\lfloor\frac{n-1}{2}\right\rfloor}(b, b)$ e se $2 \leq j \leq\left\lfloor\frac{n}{2}\right\rfloor$, seja $\rho\left(b^{j}\right)=1 / \rho\left(b^{n-j}\right)=\omega_{2}^{j} / \nu_{j-1}(b, b)$. Nesse caso, a mesma fórmula também é válida para $j=1 \mathrm{e}$ 
para $j=\left\lfloor\frac{n+1}{2}\right\rfloor$ :

$$
\rho\left(b^{\left\lfloor\frac{n+1}{2}\right\rfloor}\right)=\frac{1}{\rho\left(b^{\left\lfloor\frac{n}{2}\right\rfloor}\right)}=\frac{\nu_{\left\lfloor\frac{n}{2}\right\rfloor-1}(b, b)}{\omega_{2}^{\left\lfloor\frac{n}{2}\right\rfloor}}=\frac{\omega_{2}^{\left\lfloor\frac{n+1}{2}\right\rfloor}}{\nu_{\left\lfloor\frac{n-1}{2}\right\rfloor}(b, b)} .
$$

Também será preciso que $\nu_{j}\left(a, b^{l}\right)=\rho^{j}(a) \rho\left(b^{l}\right) / \rho\left(a^{j} b^{l}\right)$ nos seguintes casos:

- Se $1 \leq j \leq m$ e $1 \leq l \leq\lfloor(n-1) / 2\rfloor($ se $n \geq 3)$; e

- Se $1 \leq j \leq\lfloor(m+1) / 2\rfloor$ e $l=n / 2$.

Portanto, defina $\rho(a)=1 / \rho\left(a^{m-1}\right)=\rho(1)=1$ e defina

$$
\rho\left(a^{j} b^{l}\right)=\frac{1}{\rho\left(a^{m-j} b^{n-l}\right)}=\frac{\rho^{j}(a) \rho\left(b^{l}\right)}{\nu_{j}\left(a, b^{l}\right)}=\frac{\omega_{2}^{l}}{\nu_{j}\left(a, b^{l}\right) \nu_{l-1}(b, b)}
$$

nestes casos. Finalmente, para obter $\nu\left(a^{i}, b\right)=\rho\left(a^{i}\right) \rho(b) / \rho\left(a^{i} b\right)$ quando $1 \leq i \leq\lfloor m / 2\rfloor$, defina

$$
\rho\left(a^{i}\right)=\frac{1}{\rho\left(a^{m-i}\right)}=\frac{\nu\left(a^{i}, b\right) \rho\left(a^{i} b\right)}{\rho(b)} \stackrel{(4.36)}{=} \frac{\nu\left(a^{i}, b\right) \omega_{2}}{\omega_{2} \nu_{i}(a, b) \nu_{0}(b, b)}=\frac{\nu\left(a^{i}, b\right)}{\nu_{i}(a, b)} .
$$

A aplicação $\rho$ assim definida satisfaz as seguintes propriedades:

- Se $1 \leq k \leq m-1$ e $1 \leq l \leq\lfloor(n-1) / 2\rfloor$, ou se $n$ é par, $l=n / 2$ e $1 \leq k \leq\lfloor(m-1) / 2\rfloor$ então

$$
\frac{\rho(a) \rho\left(a^{k} b^{l}\right)}{\rho\left(a^{k+1} b^{l}\right)}=\frac{\omega_{2}^{l}}{\nu_{k}\left(a, b^{l}\right) \nu_{l-1}(b, b)} \frac{\nu_{k+1}\left(a, b^{l}\right) \nu_{l-1}(b, b)}{\omega_{2}^{l}}=\nu\left(a, a^{k} b^{l}\right)
$$

- Se $1 \leq i \leq\lfloor m / 2\rfloor$

$$
\frac{\rho\left(a^{i}\right) \rho(b)}{\rho\left(a^{i} b\right)}=\frac{\nu\left(a^{i}, b\right)}{\nu_{i}(a, b)} \omega_{2} \frac{\nu_{i}(a, b) \nu_{0}(b, b)}{\omega_{2}}=\nu\left(a^{i}, b\right) .
$$

- Se $n \geq 3$ e $1 \leq l \leq\lfloor(n-1) / 2\rfloor$ então

$$
\frac{\rho(b) \rho\left(b^{l}\right)}{\rho\left(b^{l+1}\right)}=\omega_{2} \frac{\omega_{2}^{l}}{\nu_{l-1}(b, b)} \frac{\nu_{l}(b, b)}{\omega_{2}^{l+1}}=\nu\left(b, b^{l}\right) .
$$

Seja $c_{m, n}=\left|S_{C_{m} \times C_{n}}\right|$ o número de $S_{3}$-órbitas efetivas do grupo $C_{m} \times C_{n}$. Note que pelo Exemplo 4.2.4, $c_{m, n}=\left|S_{C_{m}}\right|+\left|B_{1}\right|+\left|B_{2}\right|$ em que

$$
\begin{aligned}
& \left|B_{1}\right|= \begin{cases}\lfloor(m-1) / 2\rfloor m(n-1)+m(n-1) / 2, & \text { se } 2 \mid m, \\
\lfloor(m-1) / 2\rfloor m(n-1), & \text { se } 2 \nmid m,\end{cases} \\
& \left|B_{2}\right|= \begin{cases}m^{2}\left(\left|S_{C_{n}}\right|-1\right)+m+2\left|S_{C_{m}}\right|, & \text { se } n \geq 3 \text { e } 3 \mid n, \\
m^{2}\left|S_{C_{n}}\right|, & \text { se } n \geq 3 \text { e } 3 \nmid n, \\
0, & \text { se } n=2,\end{cases}
\end{aligned}
$$

e por $(3.7)$, tem-se

$$
\left|S_{C_{n}}\right|=\left|\operatorname{Bl}\left(C_{n}\right)\right|= \begin{cases}\frac{(n-1)(n-2)}{6}, & \text { se } 3 \nmid n, \\ \frac{(n-1)(n-2)+4}{6}, & \text { se } 3 \mid n .\end{cases}
$$

Corolário 4.2.6. Se $G=C_{m} \times C_{n}$, então $p M_{G \times G}(G) \simeq\left(K^{*}\right)^{c_{m, n}-\left|Q_{1}\right|-\left|Q_{2}\right|}$. 
Demonstração. Considerando $S_{C_{m} \times C_{n}}$ como no Exemplo 4.2.4, escreva

$$
\left(K^{*}\right)^{c_{m, n}}=\left\{\{x(u, v)\}_{(u, v) \in S_{C_{m} \times C_{n}}}\right\} .
$$

Pela Proposição 4.2.5, o subgrupo $L=L_{G \times G}=\left\{x \in\left(K^{*}\right)^{c_{m, n}} \mid \sigma_{x} \sim 1\right\}$ de $\left(K^{*}\right)^{c_{m, n}}$ é dado por

$$
\begin{aligned}
L=\{ & x \in\left(K^{*}\right)^{c_{m, n}} \mid x\left(a^{i}, a^{j}\right)=\frac{x\left(a, a^{i}\right) \ldots x\left(a, a^{i+j-1}\right)}{x(a, a) \ldots x\left(a, a^{j-1}\right)}, \text { para }\left(a^{i}, a^{j}\right) \in J_{1} ; \\
& x\left(a, a^{j}\right)=x\left(a, a^{m-j-1}\right), \text { para } j \in J_{2} ; x\left(a, a^{j}\right)=\frac{x(a, b) x\left(a^{j}, a b\right)}{x\left(a^{1+j}, b\right)}, \text { para } j \in J_{3} ; \\
& x\left(a^{i}, a^{k} b^{l}\right)=\frac{x\left(a, a^{i} b^{l}\right) \ldots x\left(a, a^{i+k-1} b^{l}\right) x\left(a^{i}, b^{l}\right)}{x\left(a, b^{l}\right) \ldots x\left(a, a^{k-1} b^{l}\right)}, \text { para }\left(a^{i}, a^{k} b^{l}\right) \in J_{4} ; \\
& x\left(a, a^{k} b^{l}\right)=x\left(a, a^{m-1-k} b^{n-l}\right), \operatorname{para}(k, l) \in J_{5} ; \\
& x\left(a, a^{k} b^{n / 2}\right)=x\left(a, a^{m-1-k} b^{n / 2}\right), \operatorname{para} k \in J_{6} ; \\
& x\left(a^{i}, b^{l}\right)=x\left(a^{i}, b\right) \frac{x\left(a, b^{l}\right) \ldots x\left(a, a^{i-1} b^{l}\right)}{x(a, b) \ldots x\left(a, a^{i-1} b\right)}, \operatorname{para}(i, l) \in J_{7} ; \\
& x\left(a^{i} b^{j}, a^{k} b^{l}\right)=x\left(b^{j}, b^{l}\right) \frac{x\left(a^{i}, a^{k} b^{l}\right) x\left(a^{i+k}, b^{j+l}\right)}{x\left(a^{i}, b^{j}\right) x\left(a^{i+k}, b^{l}\right)}, \operatorname{para}(i, l) \in J_{8} ; \\
& x\left(b^{j}, b^{l}\right)=\frac{x\left(b, b^{j}\right) \ldots x\left(b, b^{j+l-1}\right)}{x(b, b) \ldots x\left(b, b^{l-1}\right)}, \text { para }\left(b^{j}, b^{l}\right) \in J_{9} ; \\
& \left.x\left(b, b^{l}\right)=x\left(b, b^{n-l-1}\right), \operatorname{para} l \in J_{10}\right\}
\end{aligned}
$$

em que

$$
\begin{aligned}
J_{1} & =\left\{\left(a^{i}, a^{j}\right) \in S_{C_{m}} \mid i, j \geq 2\right\}, \\
J_{2} & =\{j \in \mathbb{N} \mid\lfloor(m+1) / 2\rfloor \leq j \leq m-3\}, \\
J_{3} & =\{j \in \mathbb{N} \mid 1 \leq j \leq\lfloor(m-1) / 2\rfloor\}, \\
J_{4} & =\left\{\left(a^{i}, a^{k} b^{l}\right) \in B_{1} \mid 2 \leq i \text { e } 1 \leq k\right\}, \\
J_{5} & =\{(k, l) \in \mathbb{N} \times \mathbb{N} \mid 1 \leq k \leq m-1 \text { e }\lfloor n / 2\rfloor+1 \leq l \leq n-1\}, \\
J_{6} & =\{k \in \mathbb{N} \mid\lfloor m / 2\rfloor \leq k \leq m-1\}, \\
J_{7} & =\{(i, l) \in \mathbb{N} \times \mathbb{N} \mid 1 \leq i \leq m / 2 \text { e } 2 \leq l \leq n / 2\}, \\
J_{8} & =\left\{\left(a^{i} b^{j}, a^{k} b^{l}\right) \in B_{2} \mid(i, k) \neq(0,0)\right\} \\
J_{9} & =\left\{\left(b^{j}, b^{l}\right) \in S_{C_{n}} \mid j, l \geq 2\right\}, \\
J_{10} & =\{l \in \mathbb{N} \mid\lfloor(n+1) / 2\rfloor \leq l \leq n-3\} .
\end{aligned}
$$

Sejam $Q_{1} \subseteq B_{1} \subseteq S_{C_{m} \times C_{n}}$ o conjunto de pares dados por (4.22), (4.23) e (4.24) e $Q_{2} \subseteq B_{2} \subseteq$ $S_{C_{m} \times C_{n}}$ o conjunto de pares dados por (4.25). Note que

$$
\left|Q_{1}\right|= \begin{cases}m\lfloor(n-1) / 2\rfloor+\lfloor m / 2\rfloor-1+\lfloor(m+1) / 2\rfloor, & \text { se } 2\lfloor n, \\ m\lfloor(n-1) / 2\rfloor+\lfloor m / 2\rfloor-1, & \text { se } 2 \nmid n,\end{cases}
$$

que

$$
\left|Q_{2}\right|= \begin{cases}\lfloor(n-1) / 2\rfloor, & \text { se } n \geq 3, \\ 0, & \text { se } n=2\end{cases}
$$

que $\left|B_{1} \backslash Q_{1}\right|=\left|J_{4}\right|+\left|J_{5}\right|+\left|J_{6}\right|+\left|J_{7}\right|$ e que $\left|B_{2} \backslash Q_{2}\right|=\left|J_{8}\right|+\left|J_{9}\right|+\left|J_{10}\right|$. Note também que $c_{m, n}-\left|Q_{1}\right|-\left|Q_{2}\right|=\left|S_{C_{m} \times C_{n}}\right|+\left|B_{1} \backslash Q_{1}\right|+\left|B_{2} \backslash Q_{2}\right|$ e, motivando-se pelas relações que descrevem 
$L$, considere o homomorfismo $\Lambda:\left(K^{*}\right)^{c_{m, n}} \rightarrow\left(K^{*}\right)^{c_{m, n}-\left|Q_{1}\right|-\left|Q_{2}\right|}$ que leva $\{x(u, v)\}_{(u, v) \in S_{C_{m} \times C_{n}}}$ em

$$
\begin{aligned}
& \left(\left(\frac{x\left(a, a^{i}\right) \ldots x\left(a, a^{i+j-1}\right)}{x\left(a^{i}, a^{j}\right) x(a, a) \ldots x\left(a, a^{j-1}\right)}\right)_{\left(a^{i}, a^{j}\right) \in J_{1}},\left(\frac{x\left(a, a^{m-j-1}\right)}{x\left(a, a^{j}\right)}\right)_{j \in J_{2}},\right. \\
& \quad\left(\frac{x(a, b) x\left(a^{j}, a b\right)}{x\left(a, a^{j}\right) x\left(a^{1+j}, b\right)}\right)_{j \in J_{3}},\left(\frac{x\left(a, a^{i} b^{l}\right) \ldots x\left(a, a^{i+k-1} b^{l}\right) x\left(a^{i}, b^{l}\right)}{x\left(a^{i}, a^{k} b^{l}\right) x\left(a, b^{l}\right) \ldots x\left(a, a^{k-1} b^{l}\right)}\right)_{\left(a^{i}, a^{k} b^{l}\right) \in J_{4}}, \\
& \quad\left(\frac{x\left(a, a^{m-1-k} b^{n-l}\right)}{x\left(a, a^{k} b^{l}\right)}\right)_{(k, l) \in J_{5}},\left(\frac{x\left(a, a^{m-1-k} b^{n / 2}\right)}{x\left(a, a^{k} b^{n / 2}\right)}\right)_{k \in J_{6}}, \\
& \quad\left(\frac{x\left(a^{i}, b\right) x\left(a, b^{l}\right) \ldots x\left(a, a^{i-1} b^{l}\right)}{x\left(a^{i}, b^{l}\right) x(a, b) \ldots x\left(a, a^{i-1} b\right)}\right)_{(i, l) \in J_{7}},\left(\frac{x\left(b^{j}, b^{l}\right) x\left(a^{i}, a^{k} b^{l}\right) x\left(a^{i+k}, b^{j+l}\right)}{x\left(a^{i} b^{j}, a^{k} b^{l}\right) x\left(a^{i}, b^{j}\right) x\left(a^{i+k}, b^{l}\right)}\right)_{(i, l) \in J_{8}}, \\
& \left.\quad\left(\frac{x\left(b, b^{j}\right) \ldots x\left(b, b^{j+l-1}\right)}{x\left(b^{j}, b^{l}\right) x(b, b) \ldots x\left(b, b^{l-1}\right)}\right)_{\left(b^{j}, b^{l}\right) \in J_{9}},\left(\frac{x\left(b, b^{n-l-1}\right)}{x\left(b, b^{l}\right)}\right)_{l \in J_{10}}\right) .
\end{aligned}
$$

Pela forma como $\Lambda$ foi construído, resulta que ele é um epimorfismo de grupos cujo núcleo é precisamente L. Consequentemente, pelo Teorema 1.5.12,

$$
p M_{G \times G}(G) \simeq \frac{\left(K^{*}\right)^{c_{m, n}}}{L} \simeq\left(K^{*}\right)^{c_{m, n}-\left|Q_{1}\right|-\left|Q_{2}\right|} .
$$

\subsection{Grupos Dicíclicos}

Para um inteiro positivo $m$, seja

$$
\operatorname{Dic}_{m}=\left\langle a, b \mid a^{2 m}=1, b^{2}=a^{m}, b^{-1} a b=a^{-1}\right\rangle .
$$

Note que todo elemento de $\mathrm{Dic}_{m}$ pode ser representado sob a forma $a^{i} b^{l}$, com $0 \leq i \leq 2 m-1 \mathrm{e}$ $l \in\{0,1\}$. Além disso, $\left(a^{k} b\right)^{-1}=a^{k+m} b$ e $\left(a^{k}\right)^{-1}=a^{2 m-k}$.

No próximo resultado, $S_{C_{2 m}}$ denota o conjunto completo de representantes das órbitas efetivas de $C_{2 m}$ escolhido em (4.6).

Lema 4.3.1. O conjunto

$$
S_{\mathrm{Dic}_{m}}=S_{C_{2 m}} \cup\left\{\left(a^{i}, a^{k} b\right) \mid 1 \leq i \leq m-1,0 \leq k \leq 2 m-1\right\} \cup\left\{\left(a^{m}, a^{k} b\right) \mid 0 \leq k \leq m-1\right\}
$$

contém exatamente um representante de cada órbita efetiva de $S_{3}$ em $\mathrm{Dic}_{m} \times \mathrm{Dic}_{m}$.

Demonstração. Conforme (1.6), tem-se $S_{3}\left(x, a^{k}\right)=S_{3}\left(a^{2 m-k}, x^{-1}\right)$, então toda órbita $S_{3}\left(x, a^{k}\right)$, com $x \in \mathrm{Dic}_{m}$, pode ser representada por um par cuja primeira coordenada é uma potência de $a$. Além disso, como $b a=a^{-1} b$, obtém-se

$$
a^{i} b a^{k} b=a^{i-1} b a^{k-1} b=a^{i-2} b a^{k-2} b=\ldots=a^{i-k} b^{2}=a^{m+i-k},
$$

e consequentemente, $S_{3}\left(a^{i} b, a^{k} b\right)=S_{3}\left(a^{i} b a^{k} b,\left(a^{k} b\right)^{-1}\right)=S_{3}\left(a^{m+i-k}, a^{k+m} b\right)$.

Como um conjunto $S_{C_{2 m}}$ (que pode ser definido por (4.18)) contém precisamente um elemento de cada $S_{3}$-órbita efetiva em $C_{2 m} \times C_{2 m}$, resta considerar os pares $\left(a^{i}, a^{k} b\right)$. Como $S_{3}\left(a^{i}, a^{k} b\right)=$ $S_{3}\left(\left(a^{i}\right)^{-1}, a^{i} a^{k} b\right)=S_{3}\left(a^{2 m-i}, a^{i+k} b\right)$, pode-se assumir que $i \leq 2 m-i$, ou seja, $i \leq m$. No caso em que $i=m$, resulta que $S_{3}\left(a^{m}, a^{k} b\right)=S_{3}\left(a^{m}, a^{m+k} b\right)$, e pode-se escolher $k \leq m-1$. De fato, $a^{m+k} b=a^{m+k-2 m} b=a^{k-m} b$ e se $m \leq k \leq 2 m-1$, então $0 \leq m-k \leq m-1$. 
Proposição 4.3.2. Seja $G=$ Dic $_{m}$, para algum número natural $m \geq 2$. Se $\sigma \in \operatorname{pm}_{G \times G}^{\prime}(G)$ e $\sigma \sim 1$ então $\sigma$ é unicamente determinado por seus valores nos pares

$$
\begin{aligned}
\left(a, a^{k} b\right), \text { em que } 0 & \leq k \leq m, \\
\left(a^{i}, b\right), \text { em que } 2 & \leq i \leq m-1,
\end{aligned}
$$

que podem ser escolhidos arbitrariamente em $K^{*}$, e também o seu valor em $\left(a^{m}, b\right)$, que deve satisfazer

$$
\sigma^{2}\left(a^{m}, b\right)=\frac{\left(\sigma_{m}(a, b)\right)^{2}}{\left(\sigma(a, b) \sigma\left(a, a^{m} b\right)\right)^{m}} .
$$

Demonstração. Seja $\sigma \in \mathrm{pm}_{G \times G}^{\prime}(G)$ e suponha que $\sigma \sim 1$. Por (4.14),

$$
\sigma\left(a^{i}, a^{k}\right)=\frac{\sigma\left(a^{i}, b\right) \sigma\left(a^{k}, a^{i} b\right)}{\sigma\left(a^{i+k}, b\right)},
$$

para quaisquer $i$ e $k$. Assim, usando o Lema 4.3.1, é claro que os valores de $\sigma$ nos pares $\left(a^{i}, a^{k}\right)$ de $S_{C_{2 m}}$ são determinados pelos seus valores em

$$
B=S_{\text {Dic }_{m}} \backslash S_{C_{2 m}}=\left\{\left(a^{i}, a^{k} b\right) \mid 1 \leq i \leq m-1,0 \leq k \leq 2 m-1\right\} \cup\left\{\left(a^{m}, a^{k} b\right) \mid 0 \leq k \leq m-1\right\} .
$$

Por (4.13), os valores de $\sigma$ naqueles pares para os quais $i \geq 2$ e $k \geq 1$ são determinados pelos valores $\sigma\left(a, a^{k} b\right)$ e $\sigma\left(a^{i}, b\right)$, em que $0 \leq k \leq 2 m-1$ e $2 \leq i \leq m$ :

$$
\sigma\left(a^{i}, a^{k} b\right)=\frac{\sigma_{i+k}(a, b) \sigma\left(a^{i}, b\right)}{\sigma_{i}(a, b) \sigma_{k}(a, b)}=\frac{\sigma\left(a, a^{i} b\right) \ldots \sigma\left(a, a^{i+k-1} b\right) \sigma\left(a^{i}, b\right)}{\sigma(a, b) \ldots \sigma\left(a, a^{k-1} b\right)} .
$$

Além disso, seja $\sigma(x, y)=\rho(x) \rho(y) \rho(x, y)^{-1}$, para alguma $\rho: G \rightarrow K^{*}$ satisfazendo (1.12) e (1.13). Assim, considerando que

$$
\sigma\left(a, a^{k} b\right) \sigma\left(a, a^{k+m} b\right)=\frac{\rho(a) \rho\left(a^{k} b\right)}{\rho\left(a^{k+1} b\right)} \frac{\rho(a) \rho\left(a^{k+m} b\right)}{\rho\left(a^{k+m+1} b\right)}=\rho^{2}(a)
$$

para qualquer $k$, resulta que se $m+1 \leq k \leq 2 m-1$ tem-se

$$
\sigma\left(a, a^{k} b\right)=\frac{\sigma(a, b) \sigma\left(a, a^{m} b\right)}{\sigma\left(a, a^{k-m} b\right)}, \text { em que } 1 \leq k-m \leq m-1 .
$$

Em outras palavras, os valores de $\sigma\left(a, a^{k} b\right)$ para $k=m+1, \ldots, 2 m-1$ são determinados pelos valores $\sigma\left(a, a^{k} b\right)$ em que $1 \leq k \leq m$. Consequentemente, os valores de $\sigma$ nos elementos de $B$ são determinados por seus valores naqueles pares de (4.37) e (4.38). Além disso, (4.39) resulta de

$$
\sigma^{2}\left(a^{m}, b\right)\left(\sigma(a, b) \sigma\left(a, a^{m} b\right)\right)^{m}=\left(\frac{\rho\left(a^{m}\right) \rho(b)}{\rho\left(a^{m} b\right)}\right)^{2}\left(\rho^{2}(a)\right)^{m}=\left(\frac{\rho^{m}(a) \rho(b)}{\rho\left(a^{m} b\right)}\right)^{2}=\left(\sigma_{m}(a, b)\right)^{2} .
$$

Agora fixe uma família $(\nu(x, y))_{(x, y) \in I}$ de elementos de $K^{*}$, indexada pela lista de pares (4.37) e (4.38) indicada na proposição, e escreva $\nu_{j}(a, b)=\nu(a, b) \nu(a, a b) \ldots \nu\left(a, a^{j-1} b\right)$, para $1 \leq j \leq m+1$. Suponha que esses valores satisfazem um análogo de (4.39), isto é, que

$$
\nu^{2}\left(a^{m}, b\right)=\frac{\left(\nu_{m}(a, b)\right)^{2}}{\left(\nu(a, b) \nu\left(a, a^{m} b\right)\right)^{m}} .
$$

O objetivo é mostrar que esses valores determinam um conjunto fator parcial $\sigma \sim 1$. Pelo Corolário 1.5.11 (ii), se for construída uma aplicação $\rho: G \rightarrow K^{*}$ que satisfaça (1.11) e (1.12) tal que $\sigma(x, y)=\rho(x) \rho(y) / \rho(x y)$, para qualquer $(x, y)$ dos que foram listados acima, então essa igualdade determinará um conjunto fator parcial $\sigma \in p m_{G \times G}^{\prime}(G)$ tal que $\sigma \sim 1$. 
Motivando-se pela equação (4.40), começe definindo $\rho(a)=1 / \rho\left(a^{2 m-1}\right)=\omega_{1}$, em que $\omega_{1}$ é tal que $\omega_{1}^{2}=\nu(a, b) \nu\left(a, a^{m} b\right)$. Defina também $\rho(1)=1$ e $\rho\left(a^{m}\right)=\omega_{1}^{m} \nu\left(a^{m}, b\right) / \nu_{m}(a, b)$, e note que por (4.41) isso implica que $\rho^{2}\left(a^{m}\right)=\omega_{1}^{2 m}\left(\nu\left(a^{m}, b\right) / \nu_{m}(a, b)\right)^{2}=1$.

Uma vez que deseja-se obter

$$
\nu\left(a^{m}, b\right)=\frac{\rho\left(a^{m}\right) \rho(b)}{\rho\left(a^{m} b\right)}=\rho\left(a^{m}\right) \rho^{2}(b),
$$

defina $\rho(b)=1 / \rho\left(a^{m} b\right)=\omega_{2}$, sendo $\omega_{2}$ tal que

$$
\omega_{2}^{2}=\frac{\nu\left(a^{m}, b\right)}{\rho\left(a^{m}\right)}=\frac{\nu\left(a^{m}, b\right) \nu_{m}(a, b)}{\omega_{1}^{m} \nu\left(a^{m}, b\right)}=\frac{\nu_{m}(a, b)}{\omega_{1}^{m}} .
$$

Depois, defina

$$
\rho\left(a^{j} b\right)=\frac{1}{\rho\left(a^{m+j} b\right)}=\frac{\rho^{j}(a) \rho(b)}{\nu_{j}(a, b)}=\frac{\omega_{1}^{j} \omega_{2}}{\nu_{j}(a, b)}
$$

para $j=1, \ldots, m-1$ e defina

$$
\rho\left(a^{j}\right)=\frac{1}{\rho\left(a^{2 m-j}\right)}=\frac{\nu\left(a^{j}, b\right) \rho\left(a^{j} b\right)}{\rho(b)}=\frac{\nu\left(a^{j}, b\right) \omega_{1}^{j} \omega_{2}}{\omega_{2} \nu_{j}(a, b)}=\frac{\nu\left(a^{j}, b\right) \omega_{1}^{j}}{\nu_{j}(a, b)},
$$

para $j=1, \ldots, m-1$ (note que a mesma fórmula também vale para $j=m$ ).

Com essas definições, segue que

$$
\begin{gathered}
\frac{\rho(a) \rho(b)}{\rho(a b)}=\omega_{1} \omega_{2} \frac{\nu(a, b)}{\omega_{1} \omega_{2}}=\nu(a, b), \\
\frac{\rho(a) \rho\left(a^{m} b\right)}{\rho\left(a^{m+1} b\right)}=\frac{\omega_{1}}{\omega_{2}} \frac{\omega_{1} \omega_{2}}{\nu(a, b)}=\frac{\omega_{1}^{2}}{\nu(a, b)}=\nu\left(a, a^{m} b\right)
\end{gathered}
$$

e, para todo $k=1, \ldots, m-1$,

$$
\frac{\rho(a) \rho\left(a^{k} b\right)}{\rho\left(a^{k+1} b\right)}=\omega_{1} \frac{\omega_{1}^{k} \omega_{2}}{\nu_{k}(a, b)} \frac{\nu_{k+1}(a, b)}{\omega_{1}^{k+1} \omega_{2}}=\frac{\nu_{k+1}(a, b)}{\nu_{k}(a, b)}=\nu\left(a, a^{k} b\right) .
$$

Além disso,

$$
\frac{\rho\left(a^{m}\right) \rho(b)}{\rho\left(a^{m} b\right)}=\frac{\omega_{1}^{m} \nu\left(a^{m}, b\right)}{\nu_{m}(a, b)} \omega_{2}^{2}=\frac{\omega_{1}^{m} \nu\left(a^{m}, b\right)}{\nu_{m}(a, b)} \frac{\nu_{m}(a, b)}{\omega_{1}^{m}}=\nu\left(a^{m}, b\right) .
$$

e para $2 \leq i \leq m-1$,

$$
\frac{\rho\left(a^{i}\right) \rho(b)}{\rho\left(a^{i} b\right)}=\frac{\nu\left(a^{i}, b\right) \omega_{1}^{i}}{\nu_{i}(a, b)} \omega_{2} \frac{\nu_{i}(a, b)}{\omega_{1}^{i} \omega_{2}}=\nu\left(a^{i}, b\right)
$$

Logo, obteve-se uma aplicação $\rho: G \rightarrow K^{*}$ com as propriedades desejadas.

Seja $d c_{m}=\left|S_{\mathrm{Dic}_{m}}\right|$ o número de $S_{3}$-órbitas efetivas do grupo Dic $m$, que pelo Lema 4.3.1 é dado pela fórmula

$$
d c_{m}= \begin{cases}\frac{(4 m-1)(4 m-2)+4}{6}, & \text { se } 3 \mid m, \\ \frac{(4 m-1)(4 m-2)}{6}, & \text { se } 3 \nmid m .\end{cases}
$$

Teorema 4.3.3. Se $G=\operatorname{Dic}_{m}$, então $p M_{G \times G}(G) \simeq\left(K^{*}\right)^{d c_{m}-2 m+1}$.

Demonstração. Considerando $\sigma_{i j}$ e $\tau_{k l}$ como em (4.5), escreva

$$
\left(K^{*}\right)^{d c_{m}}=\left\{\left(\sigma_{i j}, \tau_{k l}\right) \in\left(K^{*}\right)^{\left|S_{C_{2 m}}\right|} \times\left(K^{*}\right)^{|B|}\right\} .
$$


Pela Proposição 4.3.2, o subgrupo $L=L_{G \times G}=\left\{x \in\left(K^{*}\right)^{d c_{m}} \mid \sigma_{x} \sim 1\right\}$ de $\left(K^{*}\right)^{d c_{m}}$ é dado por

$$
\begin{gathered}
L=\left\{\left(\sigma_{i j}, \tau_{k l}\right) \in\left(K^{*}\right)^{\left|S_{C_{2 m}}\right|} \times\left(K^{*}\right)^{|B|} \mid \sigma_{i j}=\frac{\sigma_{1 i} \ldots \sigma_{1, i+j-1}}{\sigma_{11} \ldots \sigma_{1, j-1}}, \text { para }(i, j) \in J_{1} ;\right. \\
\sigma_{1 j}=\sigma_{1,2 m-j-1}, \text { para } j \in J_{2} ; \sigma_{1 j}=\frac{\tau_{10} \tau_{j 1}}{\tau_{1+j, 0}}, \text { para } j \in J_{3} ; \\
\tau_{i, k}=\frac{\left(\tau_{1, i} \ldots \tau_{1, i+k-1}\right) \tau_{i, 0}}{\tau_{1,0} \ldots \tau_{1, k-1}} \text { para }(i, k) \in J_{4} ; \\
\tau_{1, k} \tau_{1, m+k}=\tau_{1,0} \tau_{1, m}, \text { para } k \in J_{5} ; \\
\left.\tau_{m, 0}^{2}=\frac{\left(\tau_{1,0} \tau_{1,1} \ldots \tau_{1, m-1}\right)^{2}}{\left(\tau_{1,0} \tau_{1, m}\right)^{m}}\right\}
\end{gathered}
$$

em que

$$
\begin{aligned}
& J_{1}=\left\{(i, j) \in \mathbb{N} \times \mathbb{N} \mid i, j \geq 2 \text { e }\left(a^{i}, a^{j}\right) \in S_{C_{2 m}}\right\}, \\
& J_{2}=\{j \in \mathbb{N} \mid m \leq j \leq 2 m-3\}, \\
& J_{3}=\{j \in \mathbb{N} \mid 1 \leq j \leq\lfloor(2 m-1) / 2\rfloor=m-1\}, \\
& J_{4}=\left\{(i, k) \in \mathbb{N} \times \mathbb{N} \mid\left(a^{i}, a^{k} b\right) \in B, 2 \leq i \text { e } 1 \leq k\right\}, \\
& J_{5}=\{k \in \mathbb{N} \mid 1 \leq k \leq m-1\} .
\end{aligned}
$$

Seja $Q \subseteq B \subseteq S_{\text {Dic }_{m}}$ o conjunto de pares dados por (4.37) e (4.38) e note que $|Q|=2 m-1$ e que $|B \backslash Q|=\left|J_{4}\right|+\left|J_{5}\right|+1$. Escreva

$$
\left(K^{*}\right)^{d c_{m}-2 m+1}=\left\{\left(\sigma_{i j}, \tau_{k l}\right) \in\left(K^{*}\right)^{\left|S_{C_{2 m}}\right|} \times\left(K^{*}\right)^{|B \backslash Q|}\right\}
$$

e considere o homomorfismo de grupos $\Lambda:\left(K^{*}\right)^{d c_{m}} \rightarrow\left(K^{*}\right)^{d c_{m}-2 m+1}$ que leva $\left(\sigma_{i j}, \tau_{k l}\right)$ em

$$
\begin{array}{r}
\left(\left(\frac{\sigma_{i j} \sigma_{11} \ldots \sigma_{1, j-1}}{\sigma_{1 i} \ldots \sigma_{1, i+j-1}}\right)_{(i, j) \in J_{1}},\left(\frac{\sigma_{1 j}}{\sigma_{1, m-j-1}}\right)_{j \in J_{2}},\left(\frac{\sigma_{1 j} \tau_{j+1,0}}{\tau_{10} \tau_{j 1}}\right)_{j \in J_{3}},\left(\frac{\left(\tau_{1, i} \ldots \tau_{1, i+k-1}\right) \tau_{i, 0}}{\left(\tau_{1,0} \ldots \tau_{1, k-1}\right) \tau_{i, k}}\right)_{(i, k) \in J_{4}},\right. \\
\left.\left(\frac{\tau_{1,0} \tau_{1, m}}{\tau_{1, k} \tau_{1, m+k}}\right)_{k \in J_{5}}, \frac{\left(\tau_{1,0} \tau_{1,1} \ldots \tau_{1, m-1}\right)^{2}}{\tau_{m, 0}^{2}\left(\tau_{1,0} \tau_{1, m}\right)^{m}}\right) .
\end{array}
$$

É fácil verificar que $\Lambda$ é um epimorfismo cujo núcleo é precisamente $L$. Consequentemente, pelo Teorema 1.5.12, $p M_{G \times G}(G) \simeq\left(K^{*}\right)^{d c_{m}} / L \simeq\left(K^{*}\right)^{d c_{m}-2 m+1}$.

\subsection{Grupos Infinitos}

\subsubsection{Grupo Cíclico Infinito}

Denote por $\mathbb{N}$ o conjunto dos inteiros positivos e para aplicações $\sigma: \mathbb{Z} \times \mathbb{Z} \rightarrow K$ e $\rho: \mathbb{Z} \rightarrow K^{*}$, denote $\sigma_{i j}=\sigma(i, j)$ e $\rho_{i}=\rho(i)$.

Lema 4.4.1. Qualquer elemento de $p m_{\mathbb{Z} \times \mathbb{Z}}^{\prime}(\mathbb{Z})$ é unicamente determinado pelos seus valores nos pares $(i, j) \in \mathbb{N} \times \mathbb{N}$. Além disso, esses valores podem ser escolhidos arbitrariamente em $K^{*}$.

Demonstração. Observe primeiro que, para quaisquer $i, j \in \mathbb{Z}$,

$$
S_{3}(i, j)=\{(i, j),(-i-j, i),(j,-i-j),(-j,-i),(-i, i+j),(i+j,-j)\}
$$

Seja $S_{3}(i, j)$ uma órbita efetiva, ou seja, assuma que $0 \notin\{i, j, i+j\}$. Se $(i, j) \in(\mathbb{Z} \times \mathbb{Z}) \backslash(\mathbb{N} \times \mathbb{N})$, será mostrado que existe exatamente um $(m, n) \in(\mathbb{N} \times \mathbb{N}) \cap S_{3}(i, j)$. Existem alguns casos a considerar: 
- Se $i, j<0$, então $(-j,-i) \in \mathbb{N} \times \mathbb{N}$ é um representante da mesma órbita satisfazendo a propriedade desejada;

- Se $i<0<j$ e $i+j>0$ então $(-i, i+j) \in \mathbb{N} \times \mathbb{N}$. Por outro lado, se $i+j<0$ então $(j,-i-j) \in \mathbb{N} \times \mathbb{N}$.

- Se $j<0<i$ e $i+j>0$ então $(i+j,-j) \in \mathbb{N} \times \mathbb{N}$. Por outro lado, se $i+j<0$ então $(-i-j, i) \in \mathbb{N} \times \mathbb{N}$.

Finalmente, se $(i, j) \in \mathbb{N} \times \mathbb{N}$ é claro que os demais elementos de sua $S_{3}$-órbita não estão em $\mathbb{N} \times \mathbb{N}$.

Proposição 4.4.2. Se $\sigma \in p m_{\mathbb{Z} \times \mathbb{Z}}^{\prime}(\mathbb{Z})$ e $\sigma \sim 1$ então $\sigma$ é unicamente determinado por seus valores em $\{1\} \times \mathbb{N}$, que podem ser escolhidos arbitrariamente em $K^{*}$.

Demonstração. Seja $\sigma \in p m_{\mathbb{Z} \times \mathbb{Z}}^{\prime}(\mathbb{Z})$ e suponha que $\sigma \sim 1$. Isso significa que há uma aplicação $\rho: \mathbb{Z} \rightarrow K^{*}$ tal que,

$$
\sigma_{i j}=\frac{\rho_{i} \rho_{j}}{\rho_{i+j}}
$$

para todo $(i, j) \in \mathbb{Z} \times \mathbb{Z}$ e $\rho$ também satisfaz

$$
\rho_{1}=\rho_{i} \rho_{-i}=1
$$

para todo $i \in \mathbb{Z}$.

Observe que de (4.43) resulta $\sigma_{i j}=\sigma_{j i}$ e se for definido $\pi_{j}=\sigma_{11} \sigma_{12} \ldots \sigma_{1, j-1}$, para $j \geq 2$, então

$$
\pi_{j}=\frac{\rho_{1}^{j}}{\rho_{j}}
$$

e também

$$
\sigma_{i j}=\frac{\pi_{i+j}}{\pi_{i} \pi_{j}}
$$

para $i, j \geq 2$, como na prova de [21, Proposition 6.3]. Portanto, o conjunto fator parcial $\sigma$ é completamente determinado por seus valores

$$
\sigma_{11}, \sigma_{12}, \sigma_{13}, \ldots
$$

que, pelo Lema 4.4.1, correspondem a pares pertencentes a $S_{3}$-órbitas distintas.

Por outro lado, fixando $\left(\nu_{1 j}\right)_{j \in \mathbb{N}}$ arbitrariamente em $\left(K^{*}\right)^{\mathbb{N}}$, será demonstrado que esses valores determinam um conjunto fator parcial $\sigma \sim 1$. Pelo Corolário 1.5.11 (ii), é suficiente construir uma aplicação $\rho: \mathbb{Z} \rightarrow K^{*}$ satisfazendo (1.12) e tal que

$$
\nu_{1 j}=\frac{\rho_{1} \rho_{j}}{\rho_{j+1}}
$$

para $j \in \mathbb{N}$. Para $j \geq 2$, escreva $\pi_{j}=\nu_{11} \ldots \nu_{1, j-1}$. Para obter (4.45) para $j \geq 2$, defina $\rho_{1}=1 \mathrm{e}$ $\rho_{j}=1 / \rho_{-j}=1 / \pi_{j}$.

Deste modo, obtém-se uma função $\rho: \mathbb{Z} \rightarrow K^{*}$ que satisfaz (1.12) e (4.48). De fato,

$$
\sigma_{11}=\pi_{2}=\frac{\rho_{1}^{2}}{\rho_{2}}
$$

e para $j \geq 2$,

$$
\sigma_{1 j}=\frac{\pi_{j+1}}{\pi_{j}}=\frac{\frac{1}{\rho_{j+1}}}{\frac{1}{\rho_{j}}}=\frac{\rho_{j}}{\rho_{j+1}}=\frac{\rho_{1} \rho_{j}}{\rho_{j+1}} .
$$


Corolário 4.4.3. $p M_{\mathbb{Z} \times \mathbb{Z}}(\mathbb{Z}) \simeq\left(K^{*}\right)^{\mathbb{N}}$.

Demonstração. Pelo Lemma 4.4.1 e pela Proposição 4.4.2,

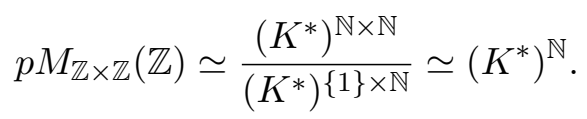

\subsubsection{Grupo Diedral Infinito}

Como um conjunto, o grupo diedral $D_{\infty}=\left\langle a, b \mid b^{2}=(a b)^{2}=1\right\rangle$ é igual a $\left\{\left(a^{i}, b^{j}\right) \mid i \in \mathbb{Z}, j \in\right.$ $\{0,1\}\}$.

Lema 4.4.4. Todo elemento de $\operatorname{pm}_{D_{\infty} \times D_{\infty}}^{\prime}\left(D_{\infty}\right)$ é unicamente determinado por seus valores nos pares do conjunto

$$
\left\{\left(a^{i}, a^{j}\right) \mid(i, j) \in \mathbb{N} \times \mathbb{N}\right\} \cup\left\{\left(a^{k}, a^{l} b\right) \mid(k, l) \in \mathbb{N} \times \mathbb{Z}\right\} .
$$

Além disso, esses valores podem ser escolhidos em $K^{*}$ arbitrariamente.

Demonstração. Análoga à prova do Lema 4.0.11.

Proposição 4.4.5. $p M_{D_{\infty} \times D_{\infty}}\left(D_{\infty}\right) \simeq\left(K^{*}\right)^{(\mathbb{N} \times \mathbb{N}) \times\left(\mathbb{N} \times \mathbb{Z}^{*}\right)}$.

Demonstração. Pelo Lema 4.4.4 as órbitas efetivas de $D_{\infty}$ podem ser indexadas pelo conjunto $(\mathbb{N} \times \mathbb{N}) \times(\mathbb{N} \times \mathbb{Z})$. Logo, pode-se considerar $\psi:\left(K^{*}\right)^{(\mathbb{N} \times \mathbb{N}) \times\left(\mathbb{N} \times \mathbb{Z}^{*}\right)} \rightarrow p M_{D_{\infty} \times D_{\infty}}\left(D_{\infty}\right)$ como sendo o epimorfismo de grupos dado por (1.16) e $x \in \operatorname{ker}(\psi)=L_{D_{\infty} \times D_{\infty}}$. Assim $\sigma=\sigma_{x} \in N_{D_{\infty} \times D_{\infty}}$ e existe uma aplicação $\rho: D_{\infty} \rightarrow K^{*}$ tal que (4.15) e (4.16) são válidas. Como no caso dos grupos diedrais finitos, é fácil verificar que $\sigma^{2}\left(a^{k}, a^{l} b\right)=\rho^{2}\left(a^{k}\right)$ para quaisquer $k$ e $l$.

Dados $j \in \mathbb{Z}$ e $x, y \in D_{\infty}$, defina

$$
\sigma_{j}(x, y)=\left\{\begin{array}{lr}
\sigma(x, y) \sigma(x, x y) \ldots \sigma\left(x, x^{j-1} y\right) & \text { se } j>0 \\
1 & \text { se } j=0 \\
\sigma\left(x, x^{-1} y\right) \sigma\left(x, x^{-2} y\right) \ldots \sigma\left(x, x^{j} y\right) & \text { se } j<0 .
\end{array}\right.
$$

Observe que no caso particular em que $x=a$ e $y=b$ a igualdade

$$
\sigma_{j}(a, b)=\frac{\rho^{|j|}(a) \rho(b)}{\rho\left(a^{j} b\right)}
$$

vale para todo $j \in \mathbb{Z}$. Utilizando (4.50), é fácil verificar que

$$
\frac{\sigma\left(a^{i}, a^{j} b\right)}{\sigma_{i+j}(a, b) \sigma\left(a^{i}, b\right)}=\frac{\rho\left(a^{i} b\right) \rho\left(a^{j} b\right)}{\rho^{|i+j|}(a)}= \begin{cases}\sigma_{i}^{-1}(a, b) \sigma_{j}^{-1}(a, b), & \text { se } i, j>0, \\ \sigma_{i}^{-1}(a, b) \sigma_{j}(a, b), & \text { se } 0<-j \leq i, \\ \sigma_{i}(a, b) \sigma_{j}^{-1}(a, b), & \text { se } 0<i \leq-j .\end{cases}
$$

Além disso, segue de (4.14) que para $i, j \geq 1$,

$$
\sigma\left(a^{i}, a^{j}\right)=\frac{\sigma\left(a^{i}, b\right) \sigma\left(a^{j}, a^{i} b\right)}{\sigma\left(a^{i+j}, b\right)} .
$$

Consequentemente, os valores de $\sigma$ em qualquer par são determinados por seus valores nos pares $\operatorname{de}\left\{\left(a^{k}, b\right) \mid k \in \mathbb{N}\right\} \cup\left\{\left(a, a^{l} b\right) \mid l \in \mathbb{Z}\right\}$. Portanto, usando o Lema 4.4.4 e considerando $\sigma_{i j}$ e $\tau_{k l}$ como 
em (4.5), e $\pi_{i}$ como em (4.8), conclui-se que

$$
\begin{gathered}
\operatorname{ker}(\psi) \subseteq R=\left\{\left(\sigma_{i j}, \tau_{k l}\right) \in\left(K^{*}\right)^{\mathbb{N} \times \mathbb{N}} \times\left(K^{*}\right)^{\mathbb{N} \times \mathbb{Z}} \mid \sigma_{i j}=\frac{\pi_{i+j}}{\pi_{i} \pi_{j}}, \text { para } i, j \geq 2 ;\right. \\
\left.\sigma_{i 1}=\sigma_{1 i}, \text { para } i \geq 2 ; \sigma_{1 j}=\frac{\tau_{10} \tau_{j 1}}{\tau_{1+j, 0}}, \text { para } j \in \mathbb{N} ; \tau_{k l}^{2}=\tau_{k 0}^{2} \text {, para }(k, l) \in \mathbb{N} \times \mathbb{Z}\right\} .
\end{gathered}
$$

Considere o grupo

$$
W=\left\{\left(\sigma_{i j}, \tau_{k l}\right) \in R \mid \tau_{k l}=\mu_{k}, \text { para }(k, l) \in \mathbb{N} \times \mathbb{Z} \text { e algum } \mu_{k} \in K^{*}\right\} .
$$

Dado $\left(\sigma_{i j}, \tau_{k l}\right) \in W$, seja $\lambda: G \rightarrow K^{*}$ a aplicação definida por

$$
\lambda\left(a^{i} b\right)=\lambda(1)=1, \text { para } i \in \mathbb{Z}
$$

e

$$
\lambda\left(a^{i}\right)=\frac{1}{\lambda\left(a^{m-i}\right)}=\mu_{i}, \text { para } i \in \mathbb{N} .
$$

Então, resulta das definições de $R$ e $W$ que

$$
\begin{gathered}
\sigma_{1 j}=\frac{\tau_{10} \tau_{j 1}}{\tau_{1+j, 0}}=\frac{\mu_{1} \mu_{j}}{\mu_{1+j}}=\lambda(a) \lambda\left(a^{j}\right) / \lambda\left(a^{1+j}\right), \text { para } j \in \mathbb{N}, \\
\sigma_{i 1}=\sigma_{1 i}=\lambda(a) \lambda\left(a^{i}\right) / \lambda\left(a^{1+i}\right), \text { para } i \geq 2 .
\end{gathered}
$$

Disto resulta que

$$
\begin{aligned}
\sigma_{i j} & =\frac{\sigma_{1 i} \ldots \sigma_{1, i+j-1}}{\sigma_{11} \ldots \sigma_{1, j-1}} \\
& =\left(\frac{\lambda(a) \lambda\left(a^{i}\right)}{\lambda\left(a^{i+1}\right)} \frac{\lambda(a) \lambda\left(a^{i+1}\right)}{\lambda\left(a^{i+2}\right)} \ldots \frac{\lambda(a) \lambda\left(a^{i+j-1}\right)}{\lambda\left(a^{i+j}\right)}\right)\left(\frac{\lambda\left(a^{2}\right)}{\lambda(a) \lambda(a)} \frac{\lambda\left(a^{3}\right)}{\lambda(a) \lambda\left(a^{2}\right)} \cdots \frac{\lambda\left(a^{j}\right)}{\lambda(a) \lambda\left(a^{j-1}\right)}\right) \\
& =\frac{\lambda^{j}(a) \lambda\left(a^{i}\right)}{\lambda\left(a^{i+j}\right)} \frac{\lambda\left(a^{j}\right)}{\lambda^{j}(a)} \\
& =\frac{\lambda\left(a^{i}\right) \lambda\left(a^{j}\right)}{\lambda\left(a^{i+j}\right)}, \text { para } i, j \geq 2
\end{aligned}
$$

e

$$
\tau_{k l}=\mu_{k}=\lambda\left(a^{k}\right) \lambda\left(a^{l} b\right) / \lambda\left(a^{k+l} b\right), \text { para }(k, l) \in \mathbb{N} \times \mathbb{Z} .
$$

Consequentemente, $W \triangleleft \operatorname{ker}(\psi)$.

Como na prova do Teorema 4.1.1, será construído um epimorfismo de grupos cujo núcleo é $W$. Para isso, seja $\Lambda:\left(K^{*}\right)^{(\mathbb{N} \times \mathbb{N}) \times(\mathbb{N} \times \mathbb{Z})} \rightarrow\left(K^{*}\right)^{(\mathbb{N} \times \mathbb{N}) \times\left(\mathbb{N} \times \mathbb{Z}^{*}\right)}$ o homomorfismo de grupos definido por

$$
\left(\sigma_{i j}, \tau_{k l}\right) \mapsto\left(\left(\frac{\sigma_{i 1}}{\sigma_{1 i}}\right)_{i \in \mathbb{N} \backslash\{1\}},\left(\frac{\sigma_{i j} \sigma_{11} \ldots \sigma_{1, j-1}}{\sigma_{1 i} \ldots \sigma_{1, i+j-1}}\right)_{i, j \in \mathbb{N} \backslash\{1\}},\left(\frac{\sigma_{1 j} \tau_{j+1,0}}{\tau_{10} \tau_{j 1}}\right)_{j \in \mathbb{N}},\left(\frac{\tau_{k l}}{\tau_{k 0}}\right)_{(k, l) \in \mathbb{N} \times \mathbb{Z}^{*}}\right) .
$$

Então $\Lambda$ é um epimorfismo, uma vez que para todo

$$
z=\left(\left(u_{i 1}\right)_{i \in \mathbb{N} \backslash\{1\}},\left(v_{i j}\right)_{i, j \in \mathbb{N} \backslash\{1\}},\left(w_{1 j}\right)_{j \in \mathbb{N}},\left(x_{k, l}\right)_{(k, l) \in \mathbb{N} \times \mathbb{Z}^{*}}\right) \in\left(K^{*}\right)^{(\mathbb{N} \times \mathbb{N}) \times\left(\mathbb{N} \times \mathbb{Z}^{*}\right)},
$$

tem-se $\Lambda\left(\sigma_{i j}, \tau_{k l}\right)=z$, em que

$$
\begin{gathered}
\tau_{k 0}=1, \text { para }(k, 0) \in \mathbb{N} \times\{0\}, \\
\tau_{k l}=x_{k l}, \text { para }(k, l) \in \mathbb{N} \times \mathbb{Z}^{*},
\end{gathered}
$$




$$
\begin{gathered}
\sigma_{1 j}=\frac{w_{1 j} \tau_{10} \tau_{j 1}}{\tau_{j+1,0}}, \text { para }(1, j) \in\{1\} \times \mathbb{N}, \\
\sigma_{i 1}=\sigma_{1, i} u_{i 1}, \text { para }(i, 1) \in \mathbb{N} \times\{1\},
\end{gathered}
$$

e

$$
\sigma_{i j}=\frac{v_{i j} \sigma_{1 i} \ldots \sigma_{1, i+j-1}}{\sigma_{11} \ldots \sigma_{1, j-1}}, \quad \text { para }(i, j) \in(\mathbb{N} \backslash\{1\}) \times(\mathbb{N} \backslash\{1\}) .
$$

Além disso, é imediato que $W=\operatorname{ker}(\Lambda)$. Dado $\mu \in \operatorname{ker}(\psi)$, resulta que

$$
\Lambda(\mu)=\left(\left(1_{i j}\right)_{(i, j) \in \mathbb{N} \times \mathbb{N}},\left(\varepsilon_{k l}\right)_{(k, l) \in \mathbb{N} \times \mathbb{Z}^{*}}\right),
$$

em que $1_{i j}=1=\varepsilon_{k l}^{2}$ para $(i, j) \in \mathbb{N} \times \mathbb{N}$ e $(k, l) \in \mathbb{N} \times \mathbb{Z}^{*}$. Em outras palavras, $\Lambda(\operatorname{ker}(\psi)) \triangleleft$ $C_{2}^{(\mathbb{N} \times \mathbb{N}) \times\left(\mathbb{N} \times \mathbb{Z}^{*}\right)}$.

Portanto,

$$
p M_{D_{\infty} \times D_{\infty}}\left(D_{\infty}\right)=\frac{\left(K^{*}\right)^{(\mathbb{N} \times \mathbb{N}) \times(\mathbb{N} \times \mathbb{Z})}}{\operatorname{ker}(\psi)} \simeq \frac{\frac{\left(K^{*}\right)^{(\mathbb{N} \times \mathbb{N}) \times(\mathbb{N} \times \mathbb{Z})}}{\operatorname{ker}(\Lambda)}}{\frac{\operatorname{ker}(\psi)}{\operatorname{ker}(\Lambda)}} \simeq \frac{\left(K^{*}\right)^{(\mathbb{N} \times \mathbb{N}) \times\left(\mathbb{N} \times \mathbb{Z}^{*}\right)}}{\Lambda(\operatorname{ker}(\psi))} \simeq\left(K^{*}\right)^{(\mathbb{N} \times \mathbb{N}) \times\left(\mathbb{N} \times \mathbb{Z}^{*}\right)}
$$

em que o último isomorfismo resulta de (4.1).

\subsection{Sugestões para Pesquisas Futuras}

No que diz respeito ao multiplicador de Schur parcial, seria interessante determinar a sua componente total, ou pelo menos uma lista de representantes para as órbitas efetivas, também para algumas outras famílias de grupos:

- Grupos abelianos livres $\mathbb{Z}^{k}, \operatorname{com} k \geq 2$;

- Produtos diretos de grupos arbitrários;

- Produtos semidiretos de grupos cíclicos finitos $C_{m} \rtimes C_{n}$, ou de forma mais geral, o produto semidireto de grupos quaisquer.

- Grupos simétricos $S_{n}$ em que $n \geq 4$; 


\section{Apêndice A}

\section{Diagramas de Domínios Elementares}

Neste apêndice, são apresentados diagramas para ilustrar o conjunto parcialmente ordenado dos domínios elementares dos grupos de ordem até 6 . No caso do grupo trivial $C_{1}=\{1\}$, há um único domínio elementar: $\mathcal{T}(1,1)$. Em cada um dos demais exemplos, será utilizada uma seta indo de $A$ para $B$ para indicar que $A \subsetneq B$.

$$
\mathcal{T}(1,1) \longrightarrow \mathcal{T}(1, a)=C_{2} \times C_{2} .
$$

Figura A.1: Ordem parcial dos dominios elementares de $C_{2}=\left\langle a \mid a^{2}=1\right\rangle$.

$$
\mathcal{T}(1,1) \longrightarrow \mathcal{T}(1, a) \longrightarrow \mathcal{T}(a, a)=C_{3} \times C_{3}
$$

Figura A.2: Ordem parcial dos dominios elementares de $C_{3}=\left\langle a \mid a^{3}=1\right\rangle$.

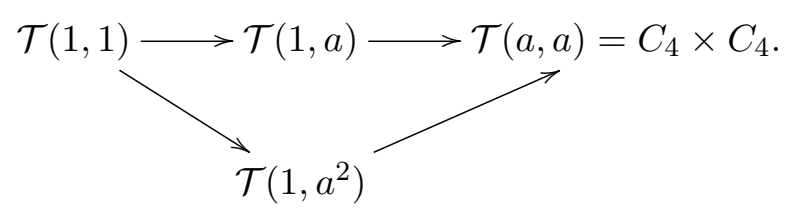

Figura A.3: Ordem parcial dos dominios elementares de $C_{4}=\left\langle a \mid a^{4}=1\right\rangle$. 


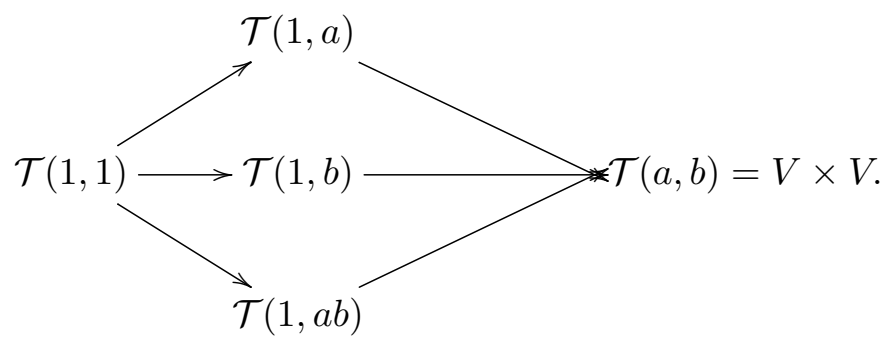

Figura A.4: Ordem parcial dos dominios elementares de $V=\left\langle a, b \mid a^{2}=b^{2}=(a b)^{2}=1\right\rangle$.

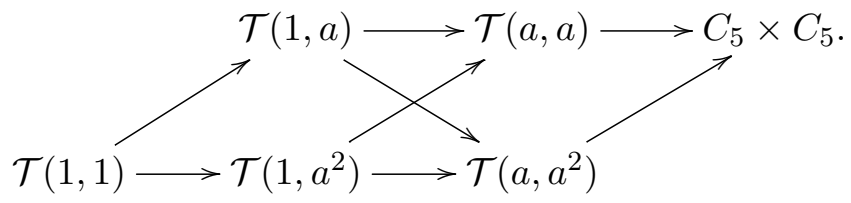

Figura A.5: Ordem parcial dos dominios elementares de $C_{5}=\left\langle a \mid a^{5}=1\right\rangle$.

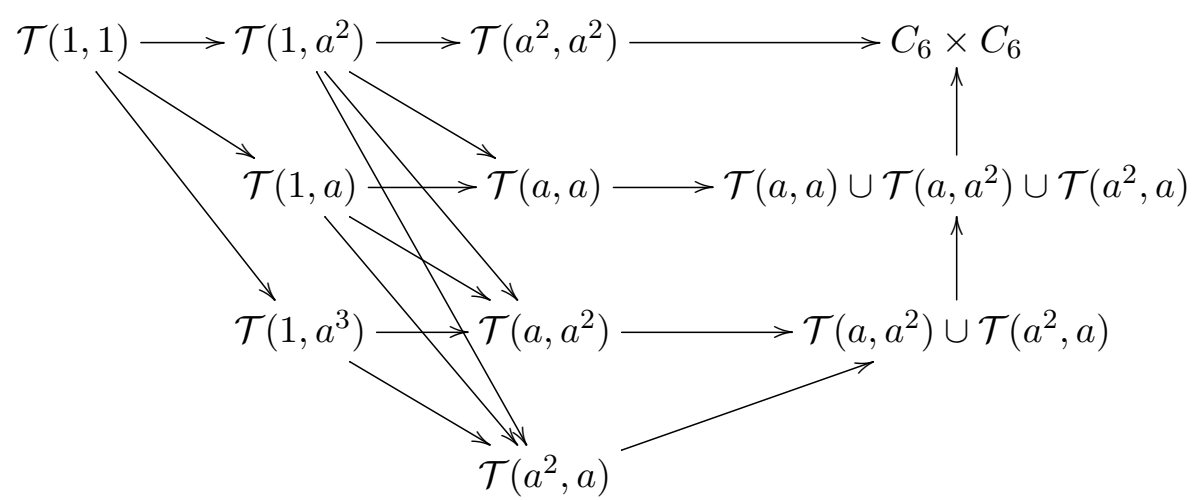

Figura A.6: Ordem parcial dos dominios elementares de $C_{6}=\left\langle a \mid a^{6}=1\right\rangle$.

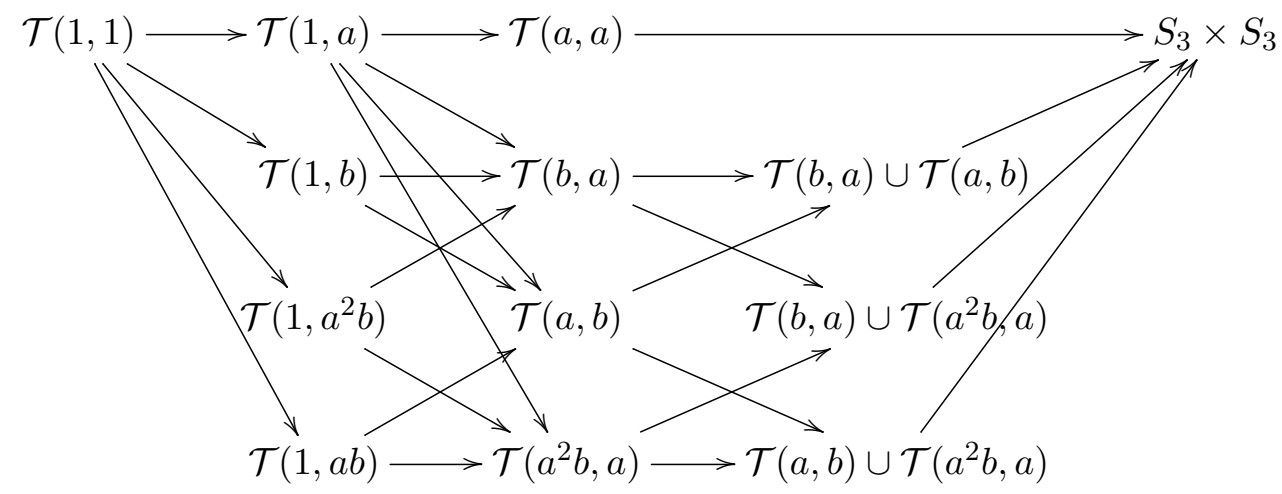

Figura A.7: Ordem parcial dos dominios elementares de $S_{3}=\left\langle a, b \mid a^{3}=b^{2}=(a b)^{2}=1\right\rangle$. 


\section{Apêndice B}

\section{$S_{3}$-órbitas Efetivas de $C_{m} \times C_{n}$}

O objetivo deste apêndice é ilustrar a lista de $S_{3}$-órbitas efetivas obtidas no Exemplo 4.2.4 por meio de exemplos concretos. Em cada uma das tabelas a seguir, é considerado um produto direto de grupos cíclicos $C_{m} \times C_{n}$, e as órbitas efetivas correspondentes são indexadas por números inteiros positivos. Os elementos de $C_{m} \times C_{n}$ aparecem tanto na primeira linha quanto na primeira coluna, e o número que aparece na interseção da linha $x$ com a coluna $y$ indica a órbita efetiva da qual o par $(x, y) \in\left(C_{m} \times C_{n}\right) \times\left(C_{m} \times C_{n}\right)$ faz parte. Os representantes das órbitas efetivas que foram escolhidos no Exemplo 4.2.4 são indicados em negrito. As posições correspondentes aos elementos das órbitas não efetivas são marcadas com um ponto.

\begin{tabular}{|c||c|c|c|c|c||c|c|c|c|c||c|c|c|c|c||}
\hline$\times$ & 1 & $a$ & $a^{2}$ & $a^{3}$ & $a^{4}$ & $b$ & $a b$ & $a^{2} b$ & $a^{3} b$ & $a^{4} b$ & $b^{2}$ & $a b^{2}$ & $a^{2} b^{2}$ & $a^{3} b^{2}$ & $a^{4} b^{2}$ \\
\hline \hline 1 &. &. &. &. &. &. &. &. &. &. &. &. &. &. &. \\
\hline$a$ &. & $\mathbf{1}$ & $\mathbf{2}$ & 1 &. & $\mathbf{3}$ & $\mathbf{4}$ & $\mathbf{5}$ & $\mathbf{6}$ & $\mathbf{7}$ & $\mathbf{8}$ & $\mathbf{9}$ & $\mathbf{1 0}$ & $\mathbf{1 1}$ & $\mathbf{1 2}$ \\
\hline$a^{2}$ &. & 2 & 2 &. & 1 & $\mathbf{1 3}$ & $\mathbf{1 4}$ & $\mathbf{1 5}$ & $\mathbf{1 6}$ & $\mathbf{1 7}$ & $\mathbf{1 8}$ & $\mathbf{1 9}$ & $\mathbf{2 0}$ & $\mathbf{2 1}$ & $\mathbf{2 2}$ \\
\hline$a^{3}$ &. & 1 &. & 2 & 2 & 16 & 17 & 13 & 14 & 15 & 21 & 22 & 18 & 19 & 20 \\
\hline$a^{4}$ &. &. & 1 & 2 & 1 & 7 & 3 & 4 & 5 & 6 & 12 & 8 & 9 & 10 & 11 \\
\hline \hline$b$ &. & 12 & 21 & 18 & 8 & $\mathbf{2 3}$ & $\mathbf{2 4}$ & $\mathbf{2 5}$ & $\mathbf{2 6}$ & $\mathbf{2 7}$ &. & 7 & 16 & 13 & 3 \\
\hline$a b$ &. & 11 & 20 & 22 & 12 & 27 & $\mathbf{2 8}$ & $\mathbf{2 9}$ & 28 & 24 & 3 & 17 & 14 & 4 &. \\
\hline$a^{2} b$ &. & 10 & 19 & 21 & 11 & 26 & 29 & 29 & 25 & 30 & 13 & 15 & 5 &. & 4 \\
\hline$a^{3} b$ &. & 9 & 18 & 20 & 10 & 25 & 28 & 26 & 31 & 31 & 16 & 6 &. & 5 & 14 \\
\hline$a^{4} b$ &. & 8 & 22 & 19 & 9 & 24 & 27 & 30 & $\mathbf{3 1}$ & $\mathbf{3 0}$ & 7 &. & 6 & 15 & 17 \\
\hline \hline$b^{2}$ &. & 7 & 16 & 13 & 3 &. & 12 & 21 & 18 & 8 & 23 & 24 & 25 & 26 & 27 \\
\hline$a b^{2}$ &. & 6 & 15 & 17 & 7 & 8 & 22 & 19 & 9 &. & 27 & 30 & 31 & 30 & 24 \\
\hline$a^{2} b^{2}$ &. & 5 & 14 & 16 & 6 & 18 & 20 & 10 &. & 9 & 26 & 31 & 31 & 25 & 28 \\
\hline$a^{3} b^{2}$ &. & 4 & 13 & 15 & 5 & 21 & 11 &. & 10 & 19 & 25 & 30 & 26 & 29 & 29 \\
\hline$a^{4} b^{2}$ &. & 3 & 17 & 14 & 4 & 12 &. & 11 & 20 & 22 & 24 & 27 & 28 & 29 & 28 \\
\hline
\end{tabular}

Tabela B.1: $S_{3}$-órbitas efetivas de $C_{5} \times C_{3}$ 


\begin{tabular}{|c||c|c|c|c||c|c|c|c||c|c|c|c|c|c|c|c||}
\hline$\times$ & 1 & $a$ & $a^{2}$ & $a^{3}$ & $b$ & $a b$ & $a^{2} b$ & $a^{3} b$ & $b^{2}$ & $a b^{2}$ & $a^{2} b^{2}$ & $a^{3} b^{2}$ & $b^{3}$ & $a b^{3}$ & $a^{2} b^{3}$ & $a^{3} b^{3}$ \\
\hline \hline 1 &. &. &. &. &. &. &. &. &. &. &. &. &. &. &. &. \\
\hline$a$ &. & $\mathbf{1}$ & 1 &. & $\mathbf{2}$ & $\mathbf{3}$ & $\mathbf{4}$ & $\mathbf{5}$ & $\mathbf{6}$ & $\mathbf{7}$ & $\mathbf{8}$ & $\mathbf{9}$ & $\mathbf{1 0}$ & $\mathbf{1 1}$ & $\mathbf{1 2}$ & $\mathbf{1 3}$ \\
\hline$a^{2}$ &. & 1 &. & 1 & $\mathbf{1 4}$ & $\mathbf{1 5}$ & 14 & 15 & $\mathbf{1 6}$ & $\mathbf{1 7}$ & 16 & 17 & $\mathbf{1 8}$ & $\mathbf{1 9}$ & 18 & 19 \\
\hline$a^{3}$ &. &. & 1 & 1 & 5 & 2 & 3 & 4 & 9 & 6 & 7 & 8 & 13 & 10 & 11 & 12 \\
\hline \hline$b$ &. & 13 & 18 & 10 & $\mathbf{2 0}$ & $\mathbf{2 1}$ & $\mathbf{2 2}$ & $\mathbf{2 3}$ & 20 & 24 & 25 & 26 &. & 5 & 14 & 2 \\
\hline$a b$ &. & 12 & 19 & 13 & $\mathbf{2 6}$ & $\mathbf{2 7}$ & $\mathbf{2 8}$ & $\mathbf{2 9}$ & 30 & 31 & 27 & 21 & 2 & 15 & 3 &. \\
\hline$a^{2} b$ &. & 11 & 18 & 12 & $\mathbf{2 5}$ & $\mathbf{3 1}$ & $\mathbf{3 2}$ & $\mathbf{3 3}$ & 32 & 28 & 22 & 34 & 14 & 4 &. & 3 \\
\hline$a^{3} b$ &. & 10 & 19 & 11 & $\mathbf{2 4}$ & $\mathbf{3 0}$ & $\mathbf{3 4}$ & $\mathbf{3 5}$ & 29 & 23 & 35 & 33 & 5 &. & 4 & 15 \\
\hline \hline$b^{2}$ &. & 9 & 16 & 6 & 20 & 29 & 32 & 30 &. & 9 & 16 & 6 & 20 & 29 & 32 & 30 \\
\hline$a b^{2}$ &. & 8 & 17 & 9 & 23 & 28 & 31 & 24 & 6 & 17 & 7 &. & 26 & 33 & 34 & 21 \\
\hline$a^{2} b^{2}$ &. & 7 & 16 & 8 & 22 & 27 & 25 & 35 & 16 & 8 &. & 7 & 25 & 35 & 22 & 27 \\
\hline$a^{3} b^{2}$ &. & 6 & 17 & 7 & 21 & 26 & 33 & 34 & 9 &. & 8 & 17 & 24 & 23 & 28 & 31 \\
\hline \hline$b^{3}$ &. & 5 & 14 & 2 &. & 13 & 18 & 10 & 20 & 21 & 22 & 23 & 20 & 24 & 25 & 26 \\
\hline$a b^{3}$ &. & 4 & 15 & 5 & 10 & 19 & 11 &. & 30 & 34 & 35 & 24 & 23 & 35 & 33 & 29 \\
\hline$a^{2} b^{3}$ &. & 3 & 14 & 4 & 18 & 12 &. & 11 & 32 & 33 & 25 & 31 & 22 & 34 & 32 & 28 \\
\hline$a^{3} b^{3}$ &. & 2 & 15 & 3 & 13 &. & 12 & 19 & 29 & 26 & 27 & 28 & 21 & 30 & 31 & 27 \\
\hline
\end{tabular}

Tabela B.2: $S_{3}$-órbitas efetivas de $C_{4} \times C_{4}$ 


\section{Referências Bibliográficas}

[1] F. Abadie, Sobre açôes parciais, fibrados de Fell, e grupoides, Ph.D. thesis, Universidade de São Paulo, 1999. 1

[2] _ Enveloping actions and Takai duality for partial actions, Journal of Functional Analysis 197 (2003), no. 1, 14-67. 1

[3] E. R. Alvares, M. M. S. Alves, and E. Batista, Partial Hopf module categories, Journal of Pure and Applied Algebra 217 (2013), no. 8, 1517-1534. 1

[4] M. M. S. Alves and E. Batista, Partial Hopf actions, partial invariants and a Morita context, Algebra and Discrete Mathematics (2009), no. 3, 1-19. 1

[5] _ Enveloping actions for partial Hopf actions, Communications in Algebra 38 (2010), no. $8,2872-2902.1$

[6] _ Globalization theorems for partial Hopf (co)actions and some of their applications, Contemporary Mathematics 537 (2011), 13-30. 1

[7] M. M. S. Alves, E. Batista, M. Dokuchaev, and A. Paques, Twisted partial actions of Hopf algebras, Israel Journal of Mathematics 197 (2013), 263-308. 1

[8] M. M. S. Alves, E. Batista, and J. Vercruysse, Partial representations of Hopf algebras, (2013), arXiv: $1309.1659 \mathrm{v} 2.1$

[9] D. Bagio, W. Cortes, M. Ferrero, and A. Paques, Actions of inverse semigroups on algebras, Communications in Algebra 35 (2007), no. 12, 3865-3874. 1

[10] D. Bagio, D. Flores, and A. Paques, Partial actions of ordered groupoids on rings, Journal of Algebra and Its Applications 9 (2010), no. 03, 501-517. 1

[11] S. Caenepeel and E. De Groot, Galois corings applied to partial Galois theory, Proc. ICMA-2004 (Kuwait), Kuwait University, 2005. 1

[12] S. Caenepeel and K. Janssen, Partial (co)actions of Hopf algebras and partial Hopf-Galois theory, Communications in Algebra 36 (2008), no. 8, 2923-2946. 1

[13] A. H. Clifford and G. B. Preston, The algebraic theory of semigroups, vol. 1, American Mathematical Society, Providence, R. I., 1961. 8, 9, 10, 11, 18, 19

[14] M. Dokuchaev, Partial actions: a survey, Contemporary Mathematics 537 (2011), 173-184. 1

[15] M. Dokuchaev and R. Exel, Associativity of crossed products by partial actions, enveloping actions and partial representations, American Mathematical Society 357 (2004), no. 5, 19311952. $1,5,6,25,27$

[16] M. Dokuchaev, R. Exel, and P. Piccione, Partial representations and partial group algebras, Journal of Algebra 226 (2000), no. 1, 505-532. 1, 2, 7, 17, 18, 21, 22, 31 
[17] M. Dokuchaev, M. Ferrero, and A. Paques, Partial actions and Galois theory, Journal of Pure and Applied Algebra 208 (2007), no. 1, 77-87. 1

[18] M. Dokuchaev and M. Khrypchenko, Partial cohomology of groups, (2013), arXiv: 1309.7069. 1

[19] M. Dokuchaev and B. Novikov, Partial projective representations and partial actions, Journal of Pure and Applied Algebra 214 (2010), 251-268. 1, 2, 13, 14

[20] _ Partial projective representations and partial actions II, Journal of Pure and Applied Algebra 216 (2012), 438-455. 1, 2, 12, 17, 26, 35

[21] M. Dokuchaev, B. Novikov, and H. Pinedo, The partial Schur multiplier of a group, Journal of Algebra 392 (2013), 199-225. 2, 13, 14, 15, 42, 45, 46, 47, 61

[22] M. Dokuchaev, B. Novikov, and G. Zholtkevych, Partial actions and automata, Algebra and Discrete Mathematics 11 (2011), no. 2, 51-63. 1

[23] M. Dokuchaev and C. Polcino Milies, Isomorphisms of partial group rings, Glasgow Mathematical Journal 46 (2004), no. 1, 161-168. 1, 7, 17

[24] M. Dokuchaev and N. Zhukavets, On finite degree partial representations of groups, Journal of Algebra 274 (2004), no. 1, 309-334. 1, 2, 17, 21, 23, 31, 32

[25] R. Exel, Circle actions on $C^{*}$-algebras, partial automorphisms, and a generalized PimsnerVoiculescu exact sequence, Journal of functional analysis 122 (1994), no. 2, 361-401. 1

[26] _ Twisted partial actions: a classification of regular $C^{*}$-algebraic bundles, Proceedings of the London Mathematical Society 74 (1997), no. 2, 417-443. 1

[27] _ Partial actions of groups and actions of inverse semigroups, Proceedings American Mathematical Society 126 (1998), 3481-3494. 1, 5, 11

[28] N. D. Gilbert, Actions and expansions of ordered groupoids, Journal of Pure and Applied Algebra 198 (2005), no. 1, 175-195. 1

[29] D. Gonçalves and D. Royer, Leavitt path algebras as partial skew group rings, (2012), arXiv: 1202.2704. 1

[30] J. Kellendonk and M. V. Lawson, Partial actions of groups, International Journal of Algebra and Computation 14 (2004), no. 1, 87. 1, 5, 11

[31] M. V. Lawson, Inverse semigroups: the theory of partial symmetries, World Scientific Publishing Company, Londres, 1998. 22

[32] K. McClanahan, K-theory for partial crossed products by discrete groups, Journal of Functional Analysis 130 (1995), no. 1, 77-117. 1

[33] M. Megrelishvili and L. Schroder, Globalization of confluent partial actions on topological and metric spaces, Topology and its Applications 145 (2004), no. 1-3, 119-145. 1, 5

[34] B. Novikov and H. Pinedo, On components of the partial Schur multiplier, Communications in Algebra 42 (2014), no. 6, 2484-2495. 2, 13

[35] J. Okniński, Semigroup Algebras, Dekker, New York, 1991. 9, 18

[36] A. Paques, V. Rodrigues, and A. Sant'ana, Galois correspondences for partial Galois Azumaya extensions, Journal of Algebra and Its Applications 10 (2011), no. 05, 835-847. 1 
[37] H. Pinedo, On elementary domains of partial projective representations of groups, Algebra and Discrete Mathematics 15 (2013), no. 1, 63-82. 31, 33, 34, 37

[38] _ A calculation of the partial Shur multiplier of S3, International Journal of Mathematics, Game Theory and Algebra 22 (2014), no. 4, 405-417. 2, 48

[39] B. Steinberg, Partial actions of groups on cell complexes, Monatshefte für Mathematik 138 (2003), no. 2, 159-170. 1

[40] M. B. Szendrei, A note on Birget-Rhodes expansion of groups, Journal of Pure and Applied Algebra 58 (1989), 93-99. 11 


\section{Índice Remissivo}

álgebra de semigrupo, 8 contraída, 8

álgebra parcial de grupo, 1, 2, 7

órbita efetiva, 13, 42, 45

ação parcial, 1, 5, 6

ajustada, 25

em um conjunto, 5

em uma álgebra, 6, 25

globalizável, 1, 6, 25

ações parciais equivalentes

ver isomorfismo de ações parciais, 6

anel de grupo skew, 6

bloco, 35,42

conjunto fator parcial, 13

conjunto suporte, 23

domínio

decomponível, 34

elementar, 31, 34, 37, 39

indecomponível, 34, 39

minimal, 35, 40

trivial, 33

fator principal, $9,10,12,17$

grupoide, $21-23$

homomorfismo parcial, 13

ideal principal, 8

idempotente primitivo, 9

isomorfismo

de ações parciais, 6

$\mathcal{J}$-classes, 8

ordenação das, 8

morfismo, 5

de ações parciais, 5

de representações parciais, 7

multiplicador de Schur parcial, 1-3, 45

componente total, 45

grupo cíclico infinito, 62 grupo diedral infinito, 62

grupos dicíclicos, 59

grupos diedrais, 48

produto de grupos cíclicos, 55

núcleo de um semigrupo, 9

órbita efetiva

representantes

grupo cíclico infinito, 60

grupo diedral infinito, 62

grupos cíclicos, 46

grupos dicíclicos, 57

grupos diedrais, 46

produto de grupos cíclicos, 52

produto direto, 51

representação parcial, 7, 23, 25

ajustada, 25

de grau finito, 23

elementar, 31

projetiva, 1, 13, 14

separadora, 25

reticulado, 11, 40

completo, 11

limitado, 11

semigrupo

0 -simples, 9

completamente 0 -simples, 9, 10

completamente semissimples, 10

completamente simples, 9

inverso, 8

$K$-cancelativo, 12

$K$-semigrupo, 12

semissimples, 10

simples, 9

semirreticulado, 11

$\mathcal{T}$-órbita, $33-35$

transversal, 31

vértice total, 39 